\title{
Relations between elliptic multiple zeta values and a special derivation algebra
}

\author{
Johannes Broedel $^{1}$, Nils Matthes ${ }^{2}$ and Oliver Schlotterer ${ }^{3}$ \\ ${ }^{1}$ Institut für Theoretische Physik, Eidgenössische Technische Hochschule Zürich \\ Wolfgang-Pauli-Strasse 27, 8093 Zürich, Switzerland \\ ${ }^{2}$ Fachbereich Mathematik, Universität Hamburg, Bundesstraße 55, D-20146 Hamburg, \\ Germany \\ ${ }^{3}$ Max-Planck-Institut für Gravitationsphysik, Albert-Einstein-Institut Am Mühlenberg \\ 1, D-14476 Potsdam, Germany \\ E-mail: jbroedel@ethz.ch,nils.matthes@uni-hamburg.de and olivers@aei.mpg.de
}

Received 10 September 2015, revised 19 December 2015

Accepted for publication 4 January 2016

Published DD MM 2016

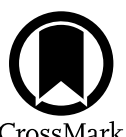

\begin{abstract}
We investigate relations between elliptic multiple zeta values (eMZVs) and describe a method to derive the number of indecomposable elements of given weight and length. Our method is based on representing eMZVs as iterated integrals over Eisenstein series and exploiting the connection with a special derivation algebra. Its commutator relations give rise to constraints on the iterated integrals over Eisenstein series relevant for eMZVs and thereby allow to count the indecomposable representatives. Conversely, the above connection suggests apparently new relations in the derivation algebra. Under https://tools.aei.mpg.de/emzv we provide relations for eMZVs over a wide range of weights and lengths.
\end{abstract}

Keywords: string-theory one-loop amplitude, elliptic multiple zeta value, iterated Integrals, derivation algebra

\section{Introduction}

While multiple zeta values (MZVs) have been a very active field of research during the last decades, their elliptic analogues have received more attention only recently. Pioneered by the work of Enriquez [1], Levin [2], Levin and Racinet [3] as well as Brown and Levin [4], many properties of elliptic multiple zeta values (eMZVs) have been identified. While mathematically interesting objects in their own right, eMZVs, the associated elliptic iterated integrals as well as related objects such as multiple elliptic polylogarithms appear in various contexts in quantum field theory and string theory. Well-known examples include the one-loop amplitude in open superstring theory [5] as well as the sunset Feynman integral and its generalizations 
[6-9]. We would also like to mention a recent application of elliptic functions to the reduction of Feynman integrals using maximal unitarity cuts [10].

Algebraic relations between usual MZVs are well understood based on their conjectural structure as a Hopf algebra comodule [11, 12]. The number of indecomposable MZVs of given weight and depth is expected to be given by the Broadhurst-Kreimer conjecture [13], which is in line with Zagier's conjecture [14] on the counting of MZVs at fixed weight. A comprehensive collection of relations among MZVs has been made available in the MZV data mine [15].

In this article, we investigate relations between eMZVs and classify their indecomposable representatives. Apart from the shuffle relations which are immediately implied by their definition as iterated integrals, eMZVs are related by Fay identities. Those identities are the generalization of partial-fraction identities, which appear in the context of usual MZVs. Both shuffle and Fay relations preserve the overall modular weight of the integrand which appears to furnish a natural analogue of the MZVs' transcendental weight. We will describe a systematic way of exploiting the combination of the two types of relations. However, the application of this method to higher weights and lengths suffers from the proliferating combinatorics of the Fay relations.

An alternative and computationally more efficient way of studying relations between eMZVs consists of employing their Fourier expansion in $q=\mathrm{e}^{2 \pi \mathrm{i} \tau}$, where $\tau$ is the modular parameter of the elliptic curve. The $q$-derivative of eMZVs is known from [1] in terms of Eisenstein series and eMZVs of lower length. Since eMZVs degenerate to MZVs at the cusp $q \rightarrow 0$ in a manner described in $[1,16]$, the supplementing boundary value is available as well. Hence, the differential equation can be integrated to yield the $q$-expansion of eMZVs recursively to-in principle-arbitrarily high order. Once the $q$-expansion of an eMZV is available up to a certain power in $q$, finding relations between eMZVs valid up to this particular power and identifying indecomposable representatives amounts to solving a linear system.

Clearly, the agreement of the respective $q$-expansions up to a certain power in $q$ is necessary but not sufficient for the validity of eMZV relations. Nevertheless, the method of $q$ expansions allows to confirm that indeed, Fay and shuffle identities comprise the entirety of eMZV relations for a variety of combinations of weights and lengths. Accordingly, this leads us to conjecture that all available relations between eMZVs are implied by Fay and shuffle identities. At lengths and weights beyond the reach of our current computer implementation of Fay and shuffle identities, the comparison of $q$-expansions gives rise to conjectural relations which nicely tie in with the algebraic considerations to be described next.

In order to overcome the shortcomings of comparing $q$-expansions of eMZVs, one uses their differential equation in $\tau$ to write eMZVs as linear combinations of iterated integrals over Eisenstein series or iterated Eisenstein integrals for short. Contrary to the definition of eMZVs, where the iterated integration is performed over coordinates of the elliptic curve, the integration in their representation via Eisenstein series is performed over the modular parameter of the elliptic curve. Similar iterated integrals, some of them involving more general modular forms, have been studied by Manin in [17]. Those integrals have been revisited by Brown [18] recently, who used them to define multiple modular values. A new feature of Brown's approach to iterated integrals of modular forms is that it allows also for an integration along paths which connect two cusps. Among other things, multiple modular values provide a conceptual explanation of the relationship between double zeta values and cusp forms [19].

The representation of eMZVs as iterated Eisenstein integrals is particularly convenient because the latter are believed to be linearly independent over the complex numbers. This has 
been tested up to the lengths and weights considered in this paper, but it remains a working hypothesis for several constructions in this work ${ }^{4}$. In particular, an analogue of the Fay relations is not known to hold for iterated Eisenstein integrals. This led to the first expectation that one can find the number of indecomposable eMZVs by enumerating all shuffle-independent iterated Eisenstein integrals.

While this idea indeed yields the correct number of indecomposable eMZVs of low length and weight, there is another effect appearing at higher weight: in the rewriting of any eMZV certain shuffle-independent iterated Eisenstein integrals occur in rigid linear combinations only. In other words, not all iterated Eisenstein integrals can be expressed in terms of eMZVs. The above rigid linear combinations in turn are implied by relations well known from a special algebra of derivations $\mathfrak{u}$ [22-25]. The patterns we find from investigating various eMZVs exactly match the available data about the derivation algebra in [24, 26]. Consequently, we turn this into a method to infer the number of indecomposable eMZVs at given weight and length. The results of this method agree perfectly with the data obtained from either shuffle and Fay relations. In addition, complete knowledge of relations in the derivation algebra leads to upper bounds on the number of indecomposable eMZVs. Those upper bounds complement the lower bounds obtained from comparing $q$-expansions. The study of $q$-expansions allows to enumerate indecomposable eMZVs of given weight and length without assuming linear independence of different iterated Eisenstein integrals, which will be discussed below.

The algebra of derivations $\mathfrak{u}$ on one side and eMZVs on the other side are linked by a differential equation for the elliptic KZB associator [22, 23] derived by Enriquez [16]. This differential equation implies upper bounds on the number of indecomposable eMZVs. Assuming these upper bounds to be attained, one can extend the knowledge about the derivation algebra $\mathfrak{u}$ substantially: we identify numerous apparently new relations up to and including depth five. Moreover, there is no conceptual bottleneck in extending the analysis to arbitrary weight and depth.

The link between eMZVs and the derivation algebra $\mathfrak{u}$ becomes particularly clear upon mapping iterated Eisenstein integrals onto words composed from non-commutative letters $g$. The viability of the bookkeeping framework introduced in section 4 relies on the linear independence of iterated Eisenstein integrals, which is not proved at this point. On the other hand, since one can always check linear independence for a finite number of iterated Eisenstein integrals directly, the empirical results made available on our eMZV webpage do not depend on the general linear independence statement.

Moreover, the rewriting of eMZVs as linear combinations of iterated Eisenstein integrals and into letters $g$ lateron bears similarities to a process, which appeared already in the context of usual MZVs. Namely, employing a conjectural isomorphism $\phi$, MZVs can be rewritten in terms of an alphabet of non-commutative letters $f$ (see [11]). However, the construction of the map $\phi$ is highly elaborate, as it requires the motivic coaction and also depends on the choice of an algebra basis for (motivic) MZVs. On the contrary, the rewriting of eMZVs in terms of iterated Eisenstein integrals is completely canonical and straightforward from the differential equation for eMZVs. On the other hand, while the number of indecomposable MZVs at given weight is determined by counting all shuffle-independent words in $f$, the corresponding problem for eMZVs requires the consideration of the non-trivial relations in the derivation algebra $\mathfrak{u}$ in addition.

4 It is expected that $\mathbb{C}$-linear independence of iterated Eisenstein integrals holds in full generality, and it can presumably be shown using the techniques introduced in [20]. We do not attempt a proof in this paper and defer this problem to future work [21]. 
In summary, the results in this work are fourfold:

- An explicit basis of irreducible eMZVs of certain weights and lengths, together with expressions of eMZVs in that basis collected on a web page https://tools.aei.mpg. de/emzv.

- The observation that Fay, shuffle and reflection relations generate all identities between eMZVs up to the weights and lengths considered.

- A general method for counting irreducible eMZVs based on iterated Eisenstein integrals and the special derivation algebra, for which we need to assume linear independence of iterated Eisenstein integrals.

- Several explicit new relations in the special derivation algebra $\mathfrak{u}$, which are collected also at https://tools.aei.mpg.de/emzv.

The article is organized as follows: section 2 starts with a small review of eMZVs and sets the stage for combining Fay and shuffle relations with the $q$-expansion, resulting in an 'observational' set of indecomposable eMZVs. Section 3 is devoted to a brief recapitulation of structures present for usual MZVs with particular emphasis on their rewriting in terms of non-commutative words. In section 4 we set up the translation of eMZVs into iterated Eisenstein integrals, investigate their properties and connect the bookkeeping of indecomposable eMZVs with the algebra of derivations $\mathfrak{u}$. In section 4.6 we describe a modified version of iterated Eisenstein integrals suitable in particular for the description of eMZVs. Several appendices are complementary to the discussion in the main text. In particular, some relations between elements of the derivation algebra are collected in appendix C.2.

\section{Relations between eMZVs}

After recalling the definition of eMZVs, we are going to explore the implications of Fay and shuffle relations as well as the method of $q$-expansions. In addition, we will describe how usual MZVs defined via

$$
\zeta_{n_{1}, n_{2}, \ldots, n_{r}} \equiv \sum_{0<k_{1}<k_{2}<\ldots<k_{r}} k_{1}^{-n_{1}} k_{2}^{-n_{2}} \ldots k_{r}^{-n_{r}}, \quad n_{r} \geqslant 2
$$

arise as constant terms of eMZVs.

\subsection{Prerequisites and definitions}

In this subsection we will briefly review elliptic iterated integrals and define eMZVs. An elaborate introduction from a string theorist's point of view is available in [5]. To get started, let us consider iterated integrals on the punctured elliptic curve $E_{\tau}^{\times}$, which is $E_{\tau} \equiv \mathbb{C} /(\mathbb{Z}+\mathbb{Z} \tau)$ with the origin removed and $\operatorname{Im}(\tau)>0$. We will frequently refer to the modular parameter $\tau$ by its exponentiated version

$$
q \equiv \mathrm{e}^{2 \pi \mathrm{i} \tau}, \quad \text { such that } \quad 2 \pi \mathrm{i} \frac{\mathrm{d}}{\mathrm{d} \tau}=-4 \pi^{2} q \frac{\mathrm{d}}{\mathrm{d} q}=-4 \pi^{2} \frac{\mathrm{d}}{\mathrm{d} \log q} .
$$

Functions $A$ of the modular parameter will be denoted by either $A(\tau)$ or $A(q)$.

2.1.1. Weighting functions. A natural collection of weighting functions for the iterated integration to be defined below is provided by the Eisenstein-Kronecker series $F(z, \alpha, \tau)$ $[4,27]$, 


$$
F(z, \alpha, \tau) \equiv \frac{\theta_{1}^{\prime}(0, \tau) \theta_{1}(z+\alpha, \tau)}{\theta_{1}(z, \tau) \theta_{1}(\alpha, \tau)},
$$

where $\theta_{1}$ is the odd Jacobi theta function and the tick denotes a derivative with respect to the first argument. The definition equation (2.3) immediately yields $F(z, \alpha, \tau)=$ $F(z+1, \alpha, \tau)$, and supplementing an additional, non-holomorphic factor lifts the quasiperiodicity of the Eisenstein-Kronecker series with respect to $z \mapsto z+\tau$ to an honest doubleperiodicity. The resulting function $\Omega(z, \alpha, \tau)$ on an elliptic curve serves as a generating series for the weighting functions $f^{(n)}(z, \tau)$ in eMZVs:

$$
\Omega(z, \alpha, \tau) \equiv \exp \left(2 \pi \mathrm{i} \alpha \frac{\operatorname{Im}(\mathrm{z})}{\operatorname{Im}(\tau)}\right) F(z, \alpha, \tau)=\sum_{n=0}^{\infty} f^{(n)}(z, \tau) \alpha^{n-1} .
$$

The functions $f^{(n)}$ are doubly periodic and alternate in their parity

$$
f^{(n)}(z+1, \tau)=f^{(n)}(z+\tau, \tau)=f^{(n)}(z, \tau), \quad f^{(n)}(-z, \tau)=(-1)^{n} f^{(n)}(z, \tau) .
$$

Their simplest instances read

$$
f^{(0)}(z, \tau)=1, \quad f^{(1)}(z, \tau)=\frac{\theta_{1}^{\prime}(z, \tau)}{\theta_{1}(z, \tau)}+2 \pi \mathrm{i} \frac{\operatorname{Im}(\mathrm{z})}{\operatorname{Im}(\tau)},
$$

and $f^{(1)}$ is in fact the only weighting function with a simple pole on the lattice $\mathbb{Z}+\mathbb{Z} \tau$ including the origin. The remaining $f^{(n)}$ with $n \neq 1$ are non-singular on the entire elliptic curve. As elaborated in [4] and section 3 of [5], the weighting functions $f^{(n)}$ can be expressed in terms of Eisenstein functions and series the latter of which will play a central rôle in the sections below.

2.1.2. Elliptic iterated integrals and eMZVs. Even though the functions $f^{(n)}$ are defined for arbitrary complex arguments $z$ and suitable for integrations along both homology cycles of the elliptic curve, we will restrict our subsequent discussion to real arguments. This is sufficient for studying eMZVs as iterated integrals over the interval $[0,1]$ on the real axis and avoids the necessity for homotopy-invariant completions of the integrands ${ }^{5}$. Hence, any integration variable and first argument of $f^{(n)}(z, \tau)$ is understood to be real.

Employing the functions $f^{(n)}$, iterated integrals on the elliptic curve $E_{\tau}^{\times}$are defined via

$$
\Gamma\left(\begin{array}{llll}
n_{1} & n_{2} & \ldots & n_{r} \\
a_{1} & a_{2} & \ldots & a_{r}
\end{array} ; z\right) \equiv \int_{0}^{z} \mathrm{~d} t f^{\left(n_{1}\right)}\left(t-a_{1}\right) \Gamma\left(\begin{array}{lll}
n_{2} & \ldots & n_{r} \\
a_{2} & \ldots & a_{r}
\end{array} ; t\right),
$$

where the recursion starts with $\Gamma(; z) \equiv 1$. The elliptic iterated integral in equation (2.7) is said to have weightw $=\sum_{i=1}^{r} n_{i}$, and the number $r$ of integrations will be referred to as its length. Beginning with the above equation, we will usually suppress the second argument $\tau$ for the weighting functions $f^{(n)}$ and the elliptic iterated integrals $\Gamma$.

Definition (2.7) implies a shuffle relation with respect to the combined letters $A_{i} \equiv \begin{aligned} & n_{i} \\ & a_{i}\end{aligned}$ describing the weighting functions $f^{\left(n_{i}\right)}\left(z-a_{i}\right)$,

$\Gamma\left(A_{1}, A_{2}, \ldots, A_{r} ; z\right) \Gamma\left(B_{1}, B_{2}, \ldots, B_{q} ; z\right)=\Gamma\left(\left(A_{1}, A_{2}, \ldots, A_{r}\right) ш\left(B_{1}, B_{2}, \ldots, B_{q}\right) ; z\right)$.

Another obvious property of elliptic iterated integrals is the reflection identity due to equation (2.5)

5 A generating series for homotopy-invariant iterated integrals is given in [4], in which the differential forms $f^{(n)}(z, \tau) \mathrm{d} z$ are accompanied by $\nu \equiv 2 \pi \mathrm{i} \frac{\operatorname{dIm}(z)}{\operatorname{Im}(\tau)}$. While any integral based upon a sequence of $\nu$ and $\mathrm{d} z$ has a unique homotopy-invariant uplift via admixtures of $f^{(n>0)}(z, \tau) \mathrm{d} z$, iterated integrals of $f^{(n)}(z, \tau) \mathrm{d} z$ allow for multiple homotopy-invariant completions via $\nu$. A thorough discussion of the issue is provided in [5]. 
$\Gamma\left(\begin{array}{llll}n_{1} & n_{2} & \ldots & n_{r} \\ a_{1} & a_{2} & \ldots & a_{r}\end{array} ; z\right)=(-1)^{n_{1}+n_{2}+\ldots+n_{r}} \Gamma\left(\begin{array}{llll}n_{r} & \ldots & n_{2} & n_{1} \\ z-a_{r} & \ldots & z-a_{2} & z-a_{1}\end{array} z\right)$.

Finally, if all the labels $a_{i}$ vanish-which, because of the periodicity of $f^{(n)}$ is equivalent to all labels $a_{i}$ being integer-we will often use the notation

$$
\Gamma\left(n_{1}, n_{2}, \ldots, n_{r} ; z\right) \equiv \Gamma\left(\begin{array}{llll}
n_{1} & n_{2} & \ldots & n_{r} \\
0 & 0 & \ldots & 0
\end{array} ; z\right) .
$$

Evaluating the elliptic iterated integrals in equation (2.10) at $z=1$ gives rise to $e M Z V \mathrm{~s}$ for short [1]:

$$
\begin{aligned}
\omega\left(n_{1}, n_{2}, \ldots, n_{r}\right) & \equiv \int_{0 \leqslant z_{i} \leqslant z_{i+1} \leqslant 1} f^{\left(n_{1}\right)}\left(z_{1}\right) \mathrm{d} z_{1} f^{\left(n_{2}\right)}\left(z_{2}\right) \mathrm{d} z_{2} \ldots f^{\left(n_{r}\right)}\left(z_{r}\right) \mathrm{d} z_{r} \\
& =\Gamma\left(n_{r}, \ldots, n_{2}, n_{1} ; 1\right),
\end{aligned}
$$

where $\ell_{\omega}=r$ is referred to as the length while $w_{\omega}=\sum_{i=1}^{r} n_{i}$ is called the weight of an eMZV. The subscript $\omega$ refers to the current $\omega$-representation of eMZVs in equation (2.11), which has a different notion of weight and length compared to the iterated Eisenstein integrals to be defined below in section 4 .

Being defined on an elliptic curve, eMZVs depend on its modular parameter $\tau$ and furnish a natural genus-one generalization of standard MZVs, which are to be reviewed in section 3 .

The shuffle relation equation (2.8) straightforwardly carries over to eMZVs

$$
\omega\left(n_{1}, n_{2}, \ldots, n_{r}\right) \omega\left(k_{1}, k_{2}, \ldots, k_{s}\right)=\omega\left(\left(n_{1}, n_{2}, \ldots, n_{r}\right) ш\left(k_{1}, k_{2}, \ldots, k_{s}\right)\right),
$$

whereas the parity property equation (2.5) of the weighting functions $f^{(n)}$ implies the reflection identity

$$
\omega\left(n_{1}, n_{2}, \ldots, n_{r-1}, n_{r}\right)=(-1)^{n_{1}+n_{2}+\ldots+n_{r}} \omega\left(n_{r}, n_{r-1}, \ldots, n_{2}, n_{1}\right) .
$$

Note that this implies the vanishing of odd-weight eMZVs with reversal-symmetric labels:

$$
\omega\left(n_{1}, n_{2}, \ldots, n_{r}\right)=0, \quad \text { if }\left(n_{1}, n_{2}, \ldots, n_{r}\right)=\left(n_{r}, \ldots, n_{2}, n_{1}\right) \text { and } \sum_{i=1}^{r} n_{i} \text { odd }
$$

Although suppressed in our notation, every eMZV is still a function of the modular parameter $\tau$ and inherits a Fourier expansion in $q$ from the restriction of $f^{(n)}$ to real arguments

$$
\omega\left(n_{1}, \ldots, n_{r}\right)=\omega_{0}\left(n_{1}, \ldots, n_{r}\right)+\sum_{k=1}^{\infty} c_{k}\left(n_{1}, \ldots, n_{r}\right) q^{k} .
$$

The $\tau$-independent quantity $\omega_{0}$ in equation (2.15) is called the constant term of $\omega$ and will be shown to consist of MZVs and integer powers of $2 \pi \mathrm{i}$ in the next subsection. We will refer to eMZVs for which $c_{k}\left(n_{1}, \ldots, n_{r}\right)=0$ for all $k \in \mathbb{N}^{+}$as constant.

2.1.3. Regularization. While the functions $f^{(n)}(z)$ are smooth for $n \neq 1$, the function $f^{(1)}(z)$ in equation (2.6) diverges as $\frac{1}{z}$ and $\frac{1}{z-1}$ for $z \rightarrow 0$ and $z \rightarrow 1$, respectively. Hence, eMZVs $\omega\left(n_{1}, \ldots, n_{r}\right)$ with $n_{1}=1$ or $n_{r}=1$ are $a$ priori divergent, and require a regularization process similar to shuffle regularization for MZVs [1] (see also [21]). A natural choice at genus one is to modify the integration region in equation (2.7) by a small $\varepsilon>0$,

$$
\int_{\varepsilon \leqslant z_{i} \leqslant z_{i+1} \leqslant z-\varepsilon} f^{\left(n_{1}\right)}\left(z_{1}-a_{1}\right) \mathrm{d} z_{1} f^{\left(n_{2}\right)}\left(z_{2}-a_{2}\right) \mathrm{d} z_{2} \ldots f^{\left(n_{r}\right)}\left(z_{r}-a_{r}\right) \mathrm{d} z_{r},
$$


and to expand the integral as a polynomial in $\log (-2 \pi \mathrm{i} \varepsilon)$. Hereby the branch of the logarithm is chosen such that $\log (-\mathrm{i})=-\frac{\pi \mathrm{i}}{2}$. The regularized value of equation (2.16) is then defined to be the constant term in the $\varepsilon$-expansion. The factor $-2 \pi \mathrm{i}$ in the expansion parameter $\log (-2 \pi \mathrm{i} \varepsilon)$ ensures that the limit $\tau \rightarrow \mathrm{i} \infty$ does not introduce any logarithms, and that eMZVs degenerate to MZVs upon setting $z=1$ in equation (2.16). For later reference, we will call eMZVs of the form $\omega\left(1, n_{2}, \ldots\right)$ or $\omega\left(\ldots, n_{r-1}, 1\right)$ divergent.

For the enumeration of eMZVs, we have employed an infinite alphabet, consisting of the non-negative integers $0,1,2, \ldots$ equation (2.11). There is another way of carrying out this enumeration, which uses a two-letter alphabet instead [4]. The two-letter alphabet descends from a construction of eMZVs via homotopy-invariant iterated integrals. Since every eMZV in the infinite alphabet can be rewritten as an eMZV in the two-letter alphabet and vice-versa, one does not lose information by choosing to work with one alphabet or the other.

\subsection{Fay and shuffle relations}

In this subsection, we analyze relations among eMZVs defined in equation (2.11) and gather examples of indecomposable eMZVs. A set of indecomposable eMZVs of weight $w_{\omega}$ and length $\ell_{\omega}$ is a minimal set of eMZVs such that any other eMZV of the same weight and length can be expressed as a linear combination of elements from this set and

- products of eMZVs with strictly positive weights,

- eMZVs of lengths smaller than $\ell_{\omega}$ or weight lower than $w_{\omega}$,

where the coefficients comprise MZVs (including rational numbers) and integer powers of $2 \pi \mathrm{i}$. After exploring the consequences of shuffle and reflection identities equations (2.12) and (2.13), Fay identities are discussed as a genus-one analogue of the partial-fraction identities among products of $(z-a)^{-1}$, which arise from the differential forms $\operatorname{dlog}(z-a)$. The weight of eMZVs is preserved under all these identities whereas the length obviously varies in Fay and shuffle relations. In contradistinction to usual MZVs, the availability of $f^{(0)} \equiv 1$ as a weighting function yields an infinite number of eMZVs for a certain weight, so the counting of indecomposable eMZVs must be performed at fixed length and weight.

2.2.1. Examples of constant eMZVs. The simplest examples of the eMZVs defined in equation (2.11) are of length one:

$$
\omega\left(n_{1}\right)=\left\{\begin{array}{cl}
-2 \zeta_{n_{1}} & : n_{1} \text { even } \\
0 & : n_{1} \text { odd }
\end{array} .\right.
$$

The underlying single integration over the interval $[0,1]$ picks up the constant term in the $q$ expansion of $f^{(n)}$ (see section 3.3 of [5]) and yields the constants in equation (2.17) with regularized value $\zeta_{0}=-\frac{1}{2}$ in $\omega(0)=1$.

Another distinction between even and odd labels $n_{i}$ occurs at length $\ell_{\omega}=2$. The union of shuffle and reflection identities equations (2.12) and (2.13) contains more independent relations for even total weight than for odd weight, and the eMZVs are then completely determined by equation (2.17):

$$
\left.\omega\left(n_{1}, n_{2}\right)\right|_{n_{1}+n_{2} \text { even }}=\left\{\begin{array}{ll}
2 \zeta_{n_{1}} \zeta_{n_{2}} & : n_{1}, n_{2} \text { even } \\
0 & : n_{1}, n_{2} \text { odd }
\end{array} .\right.
$$

For eMZVs of odd total weight, on the other hand, shuffle and reflection relations at length two coincide, and $\omega\left(n_{1}, n_{2}\right)$ are no longer bound to be constant. This correlation between 
$(-1)^{w_{\omega}}$ and the length will be turned into a general rule in the next paragraph. In addition, there are also constant eMZVs, which make their appearance only at sufficiently high length. For example, $\zeta_{3}$ is identified in equation (2.36) to be an eMZV of weight 3 and length 4 . One can show that all constant eMZVs evaluate to products of MZVs and integer powers of $2 \pi \mathrm{i}$, see proposition 5.3 of [1].

2.2.2. Interesting and boring eMZVs. The lack of a $\tau$-dependence for eMZVs $\omega\left(n_{1}, n_{2}\right)$ of even weight can be viewed as the analogue of the vanishing of $\omega\left(n_{1}\right)$ for odd weight as observed in equation (2.17). The general pattern is as follows: whenever weight and length of an eMZV have the same parity (i.e. $(-1)^{w_{\omega}}=(-1)^{\ell_{\omega}}$ ), shuffle and reflection identities equations (2.12) and (2.13) allow to determine this eMZV in terms of eMZVs of lower length. Novel indecomposable eMZVs can only occur for opposite parity $(-1)^{w_{\omega}}=-(-1)^{\ell_{\omega}}$ such as the odd-weight $\omega\left(n_{1}, n_{2}\right)$. Accordingly, an eMZV $\omega\left(n_{1}, n_{2}, \ldots, n_{r}\right)$ is called interesting, if the combination $w_{\omega}+\ell_{\omega}$ of weight and length is odd, otherwise we refer to it as boring.

Boring eMZVs at length $\ell_{\omega}=3$ can arise from four different choices of even and odd labels. For those, the shuffle identities equation (2.12) allow to reduce them to interesting eMZVs at length two. Explicitly, we have

$$
\begin{aligned}
& \omega\left(o_{1}, o_{2}, o_{3}\right)=0 \\
& \omega\left(e_{1}, e_{2}, o_{3}\right)=-\zeta_{e_{1}} \omega\left(e_{2}, o_{3}\right) \\
& \omega\left(e_{1}, o_{2}, e_{3}\right)=-\zeta_{e_{1}} \omega\left(o_{2}, e_{3}\right)-\zeta_{e_{3}} \omega\left(e_{1}, o_{2}\right) \\
& \omega\left(o_{1}, e_{2}, e_{3}\right)=-\zeta_{e_{3}} \omega\left(o_{1}, e_{2}\right),
\end{aligned}
$$

where $e_{i}$ and $o_{i}$ refer to even and odd labels, respectively. Similarly, boring eMZVs at length $\ell_{\omega}=4$ come in the following (reflection-independent) classes:

$$
\begin{aligned}
\omega\left(o_{1}, o_{2}, o_{3}, o_{4}\right) & =0 \\
\omega\left(e_{1}, e_{2}, e_{3}, e_{4}\right) & =-2 \zeta_{e_{1}} \zeta_{e_{2}} \zeta_{e_{3}} \zeta_{e_{4}}-\zeta_{e_{4}} \omega\left(e_{1}, e_{2}, e_{3}\right)-\zeta_{e_{1}} \omega\left(e_{2}, e_{3}, e_{4}\right) \\
\omega\left(o_{1}, o_{2}, e_{3}, e_{4}\right) & =-\zeta_{e_{4}} \omega\left(o_{1}, o_{2}, e_{3}\right) \\
\omega\left(o_{1}, e_{2}, o_{3}, e_{4}\right) & =\frac{1}{2} \omega\left(o_{1}, e_{2}\right) \omega\left(o_{3}, e_{4}\right)-\zeta_{e_{4}} \omega\left(o_{1}, e_{2}, o_{3}\right) \\
\omega\left(o_{1}, e_{2}, e_{3}, o_{4}\right) & =\frac{1}{2} \omega\left(o_{1}, e_{2}\right) \omega\left(e_{3}, o_{4}\right) \\
\omega\left(e_{1}, o_{2}, o_{3}, e_{4}\right) & =\frac{1}{2} \omega\left(e_{1}, o_{2}\right) \omega\left(o_{3}, e_{4}\right)-\zeta_{e_{1}} \omega\left(o_{2}, o_{3}, e_{4}\right)-\zeta_{e_{4}} \omega\left(e_{1}, o_{2}, o_{3}\right) .
\end{aligned}
$$

Although becoming more involved for higher length, the distinction of cases as well as the decomposition of boring eMZVs can be cast into a nice form, as is explained in appendix A.1. Below, however, we will be concerned with interesting eMZVs mostly. Note that the vanishing of eMZVs with only odd entries is true at all lengths,

$$
\omega\left(o_{1}, o_{2}, \ldots, o_{r}\right)=0 .
$$

2.2.3. Fay relations among $f^{(n)}$ and elliptic iterated integrals. While reflection and shuffle identities preserve the partition of the modular weight among the integrated $f^{\left(n_{i}\right)}$, so-called Fay relations mix eMZVs involving different values of $n_{i}$. They can be traced back to the Fay identity of their generating series equation (2.4) [4] 


$$
\begin{aligned}
\Omega\left(z_{1}, \alpha_{1}, \tau\right) \Omega\left(z_{2}, \alpha_{2}, \tau\right)= & \Omega\left(z_{1}, \alpha_{1}+\alpha_{2}, \tau\right) \Omega\left(z_{2}-z_{1}, \alpha_{2}, \tau\right) \\
& +\Omega\left(z_{2}, \alpha_{1}+\alpha_{2}, \tau\right) \Omega\left(z_{1}-z_{2}, \alpha_{1}, \tau\right),
\end{aligned}
$$

which is valid for any complex $z_{1}, z_{2}$ and follows from the Fay trisecant equation [28]. Relations among $f^{(n)}$ can be read off from equation (2.22) by isolating monomials in $\alpha_{1}, \alpha_{2}[5]$

$$
\begin{aligned}
f^{\left(n_{1}\right)}(t-x) f^{\left(n_{2}\right)}(t)= & -(-1)^{n_{1}} f^{\left(n_{1}+n_{2}\right)}(x)+\sum_{j=0}^{n_{2}}\left(\begin{array}{c}
n_{1}-1+j \\
j
\end{array}\right) f^{\left(n_{2}-j\right)}(x) f^{\left(n_{1}+j\right)}(t-x) \\
& +\sum_{j=0}^{n_{1}}\left(\begin{array}{c}
n_{2}-1+j \\
j
\end{array}\right)(-1)^{n_{1}+j} f^{\left(n_{1}-j\right)}(x) f^{\left(n_{2}+j\right)}(t)
\end{aligned}
$$

The simplest instance of these Fay relations can be viewed as a genus-one counterpart of partial-fraction relations such as $\frac{1}{t x}=\frac{1}{x(t-x)}+\frac{1}{t(x-t)}$ :

$$
\begin{aligned}
f^{(1)}(t-x) f^{(1)}(t)= & f^{(1)}(t-x) f^{(1)}(x)-f^{(1)}(t) f^{(1)}(x) \\
& +f^{(2)}(t)+f^{(2)}(x)+f^{(2)}(t-x) .
\end{aligned}
$$

The Fay relations equation (2.23) are a very powerful tool for rearranging the elliptic iterated integrals in equation (2.7). Together with the derivatives of $\Gamma$ with respect to their argument $z$ and labels $a_{i}$ [5], they allow for example to recursively remove any appearance of $a_{i}=z$ in the label of an iterated integral, e.g.

$$
\begin{aligned}
& \Gamma\left(\begin{array}{llll}
n_{1} & n_{2} & \ldots & n_{r} \\
z & 0 & \ldots & 0
\end{array} ; z\right)=(-1)^{r} \zeta_{r} \prod_{j=1}^{r} \delta_{n_{j}, 1}-(-1)^{n_{1}} \Gamma\left(\begin{array}{llllll}
n_{1}+n_{2} & 0 & n_{3} & \ldots & n_{r} \\
0 & 0 & 0 & \ldots & 0
\end{array} ; z\right) \\
& \quad+\sum_{j=0}^{n_{1}}(-1)^{n_{1}+j}\left(\begin{array}{c}
n_{2}-1+j \\
j
\end{array}\right) \Gamma\left(\begin{array}{lllll}
n_{1}-j & n_{2}+j & n_{3} & \ldots & n_{r} \\
0 & 0 & 0 & \ldots & 0
\end{array} ;\right) \\
& \quad+\sum_{j=0}^{n_{2}}\left(\begin{array}{c}
n_{1}-1+j \\
j
\end{array}\right) \int_{0}^{z} \mathrm{~d} t f^{\left(n_{2}-j\right)}(t) \Gamma\left(\begin{array}{lllll}
n_{1}+j & n_{3} & \ldots & n_{r} \\
t & 0 & \ldots & 0
\end{array}\right), t
\end{aligned}
$$

see appendix A.2 for a generalization to multiple appearances of $a_{i}=z$. The zeta value $\zeta_{r}$ in the first line of equation (2.25) stems from the limit $z \rightarrow 0$ of the left-hand side for which $f^{(1)}(z)$ can be approximated by $\frac{1}{z}$ [5]. Note that the Kronecker-deltas $\delta_{n_{j}, 1}$ ensure that the notions of weights for MZVs and elliptic iterated integrals are compatible in equation (2.25).

2.2.4. Fay relations among eMZVs. A rich class of eMZV relations can be inferred from the limit $z \rightarrow 1$ of equation (2.25). On the left hand side, periodicity of $f^{(n)}$ w.r.t. $z \rightarrow z+1$ leads to

$$
\lim _{z \rightarrow 1} \Gamma\left(\begin{array}{ll}
n_{1} & n_{2} \ldots n_{r} \\
z & 0 \ldots 0
\end{array} ; z\right)=\omega\left(n_{r}, \ldots, n_{2}, n_{1}\right), \quad n_{1} \neq 1 \text { or } n_{2} \neq 1,
$$

where cases with $n_{1}=n_{2}=1$ require an additional treatment of the poles of the associated $f^{(1)}$ and are therefore excluded ${ }^{6}$. By equation (2.11), the elliptic iterated integrals on the righthand side reduce to eMZVs under $z \rightarrow 1$ once the recursion equation (2.25) has been applied iteratively to remove any appearance of the argument from the labels. At length two, the resulting eMZV relation is

${ }^{6}$ For cases with $n_{1}=1$ and $n_{2} \neq 1$, we could not prove the general absence of extra contributions from the poles of $f^{(1)}$. However, the validity of equation (2.26) in these cases has been thoroughly tested to lengths $r \leqslant 6$ using the methods in section 2.3. Hence, equation (2.26) at $n_{1}=1$ and $n_{2} \neq 1$ with general $r$ remains a well-tested conjecture. 


$$
\begin{aligned}
\omega\left(n_{2}, n_{1}\right)= & -(-1)^{n_{1}} \omega\left(0, n_{1}+n_{2}\right)+\sum_{j=0}^{n_{2}}\left(\begin{array}{c}
n_{1}-1+j \\
j
\end{array}\right)(-1)^{n_{1}+j} \omega\left(n_{1}+j, n_{2}-j\right) \\
& +\sum_{j=0}^{n_{1}}\left(\begin{array}{c}
n_{2}-1+j \\
j
\end{array}\right)(-1)^{n_{1}+j} \omega\left(n_{2}+j, n_{1}-j\right), \quad n_{1} \neq 1 \text { or } n_{2} \neq 1,
\end{aligned}
$$

and length three requires two applications of the recursion in equation (2.25):

$$
\begin{aligned}
& \omega\left(n_{3}, n_{2}, n_{1}\right)=\zeta_{2} \sum_{j=0}^{n_{2}} \delta_{n_{3}, 1} \delta_{n_{1}+j, 1}\left(\begin{array}{c}
n_{1}-1+j \\
j
\end{array}\right) \omega\left(n_{2}-j\right) \\
& -(-1)^{n_{1}} \omega\left(n_{3}, 0, n_{1}+n_{2}\right)+\sum_{j=0}^{n_{1}}(-1)^{n_{1}+j}\left(\begin{array}{c}
n_{2}-1+j \\
j
\end{array}\right) \omega\left(n_{3}, n_{2}+j, n_{1}-j\right) \\
& +\sum_{j=0}^{n_{2}}\left(\begin{array}{c}
n_{1}-1+j \\
j
\end{array}\right) \sum_{k=0}^{n_{3}}(-1)^{n_{1}+j+k}\left(\begin{array}{c}
n_{1}+j-1+k \\
k
\end{array}\right) \omega\left(n_{1}+j+k, n_{3}-k, n_{2}-j\right) \\
& +\sum_{j=0}^{n_{2}}\left(\begin{array}{c}
n_{1}-1+j \\
j
\end{array}\right) \sum_{k=0}^{n_{1}+j}(-1)^{n_{1}+j+k}\left(\begin{array}{c}
n_{3}-1+k \\
k
\end{array}\right) \omega\left(n_{3}+k, n_{1}+j-k, n_{2}-j\right) \\
& -\sum_{j=0}^{n_{2}}(-1)^{n_{1}+j}\left(\begin{array}{c}
n_{1}-1+j \\
j
\end{array}\right) \omega\left(0, n_{1}+n_{3}+j, n_{2}-j\right), \quad n_{1} \neq 1 \text { or } n_{2} \neq 1 .
\end{aligned}
$$

It is straightforward to derive higher-length relations (involving any $\zeta_{r}$ with $2 \leqslant r \leqslant \ell_{\omega}-1$ ) from further iterations of equation (2.25) in the limit $z \rightarrow 1$. The exclusion of $n_{1}=n_{2}=1$ suppresses $\zeta_{2}$ in equation (2.27) and $\zeta_{3}$ in equation (2.28), and, more generally, the appearance of $\zeta_{r}$ is relegated to eMZV relations of length $r+1$. By the relations derived from $\Gamma\left(\begin{array}{lllllll}n_{1} & n_{2} & \ldots & n_{k} & n_{k+1} & \ldots & n_{r} \\ z & z & \ldots & z & 0 & \ldots & 0\end{array} ; z\right)$ in appendix A.2, analogous statements apply to generic MZVs, and any MZV will appear in the rewriting of some $\Gamma\left(\begin{array}{llll}n_{1} & n_{2} & \ldots & n_{r} \\ a_{1} & a_{2} & \ldots & a_{r}\end{array} ; z\right)$ with appropriate combinations of $a_{j} \in\{0, z\}$.

2.2.5. Combining shuffle and Fay relations. Shuffle relations reduce boring eMZVs to interesting eMZVs of lower length, see for instance equations (2.18) and (2.19). At first glance, this appears to attribute more significance to Fay relations among interesting eMZVs, e.g. equation (2.27) at odd $n_{1}+n_{2}$ and equation (2.28) at even $n_{1}+n_{2}+n_{3}$. The former yields length-two relations such as

$$
\omega(0,5)=\omega(2,3), \quad \omega(3,4)=-2 \omega(0,7)+\omega(2,5),
$$

which by themselves leave $1+\left\lfloor\frac{1}{3}\left(n_{1}+n_{2}\right)\right\rfloor$ eMZVs at length $\ell_{\omega}=2$ and weight $w_{\omega}=n_{1}+n_{2}$ independent [29]. However, Fay relations equation (2.28) among boring eMZVs at length three turn out to contain additional information about interesting $\omega\left(n_{1}, n_{2}\right)$. For example, writing equation (2.28) with $\left(n_{1}, n_{2}, n_{3}\right)=(1,0,2)$,

$$
\omega(0,3,0)-\omega(1,2,0)-\omega(2,0,1)+\omega(3,0,0)=0,
$$

followed by a shuffle-reduction of the boring eMZVs via equation (2.19) yields the lengthtwo relation

$$
\omega(1,2)=2 \zeta_{2} \omega(0,1)-\omega(0,3)
$$


This relation would be inaccessible from Fay relations at length two and identifies $\omega(0,3)$ to be the unique indecomposable $\omega\left(n_{1}, n_{2}\right)$ at weight three, which is short of the above $1+\left\lfloor\frac{1}{3}\left(n_{1}+n_{2}\right)\right\rfloor$ counting. Hence-when combined with shuffle-relations-Fay relations among boring eMZVs at length $\ell_{\omega}+1$ provide more information than their counterparts among interesting eMZVs at length $\ell_{\omega}$. The need for Fay relations at length $\ell_{\omega}+1$ to classify indecomposable eMZVs at length $\ell_{\omega}$ is reminiscent of double-shuffle relations among MZVs. For example, the relation

$$
\zeta_{5,7}=\frac{14}{9} \zeta_{3,9}+\frac{28}{3} \zeta_{5} \zeta_{7}-\frac{121285}{12438} \zeta_{12}
$$

is inaccessible from double-shuffle relations of depth two and requires higher-depth input [19].

2.2.6. Indecomposable eMZVs. By applying the shuffle-reduction equation (2.19) to higherweight instances of the length-three Fay relation equation (2.28), any length-two eMZV can be expressed in terms of products of $\zeta_{2 k}$ and $\omega(0,2 n-1)$ :

$$
\begin{aligned}
\left.\omega\left(n_{1}, n_{2}\right)\right|_{n_{1}+n_{2} \text { odd }}= & (-1)^{n_{1}} \omega\left(0, n_{1}+n_{2}\right)+2 \delta_{n_{1}, 1} \zeta_{n_{2}} \omega(0,1)-2 \delta_{n_{2}, 1} \zeta_{n_{1}} \omega(0,1) \\
& +2 \sum_{p=1}^{\left\lceil\frac{1}{2}\left(n_{2}-3\right)\right.}\left(\begin{array}{c}
n_{1}+n_{2}-2 p-2 \\
n_{1}-1
\end{array}\right) \zeta_{n_{1}+n_{2}-2 p-1} \omega(0,2 p+1) \\
& -2 \sum_{p=1}^{\left\lceil\frac{1}{2}\left(n_{1}-3\right)\right.}\left(\begin{array}{c}
n_{1}+n_{2}-2 p-2 \\
n_{2}-1
\end{array}\right) \zeta_{n_{1}+n_{2}-2 p-1} \omega(0,2 p+1),
\end{aligned}
$$

which implies that no eMZVs at length two other than $\omega(0,2 n-1)$ are indecomposable. This relation can be straightforwardly proven using the techniques of section 2.3.

Accordingly, the richest source of relations between interesting eMZVs at length three are the length-four Fay relations at even weight together with the shuffle reduction equation (2.20) of the boring eMZVs therein. The indecomposable eMZVs can be chosen to include $\omega(0,0,2 n)$ by analogy with equation (2.33), and additional indecomposable eMZVs such as $\omega(0,3,5)$ occur at weight $w_{\omega} \geqslant 8$, e.g.

$$
\begin{aligned}
\omega(1,1,2)= & \frac{13}{12} \zeta_{4}-\zeta_{2} \omega(0,1)^{2}+\omega(0,1) \omega(0,3)+3 \zeta_{2} \omega(0,0,2)-\frac{1}{2} \omega(0,0,4) \\
\omega(0,6,2)= & -\frac{21}{2} \zeta_{8}+2 \omega(0,3) \omega(0,5) \\
& -14 \zeta_{6} \omega(0,0,2)-6 \zeta_{4} \omega(0,0,4)-\frac{9}{2} \omega(0,0,8)-\frac{2}{5} \omega(0,3,5) .
\end{aligned}
$$

Similarly, the set of indecomposable length-three eMZVs at weights ten and twelve can be chosen as $\{\omega(0,0,10), \omega(0,3,7)\}$ and $\{\omega(0,0,12), \omega(0,3,9)\}$, respectively. The weighttwelve relation

$$
\begin{aligned}
\omega(0,5,7)= & -140 \zeta_{10} \omega(0,0,2)-14 \zeta_{8} \omega(0,0,4)+\frac{28}{3} \omega(0,5) \omega(0,7) \\
& -\frac{119}{6} \omega(0,0,12)+\frac{14}{9} \omega(0,3,9)-\frac{550396}{6219} \zeta_{12}
\end{aligned}
$$

will play an essential rôle later on. 
While even-weight single MZVs are special cases of length-one eMZVs by equation (2.17), odd MZVs do not show up in any relation for an eMZV of $\ell_{\omega} \leqslant 3$. When applying the above procedure to higher lengths, $\zeta_{3}$ is identified to be an eMZV by length-four relations such as

$$
\omega(0,1,2,0)=\frac{1}{4} \omega(0,3)-\frac{5}{2} \omega(0,0,0,3)-\frac{\zeta_{3}}{4} .
$$

The appearance of $\zeta_{3}$ in eMZV relations at length $\ell_{\omega}=4,5$ is governed by equation (2.26) at $r=4,5$, and similar relations are expected to hold for any odd single zeta value by equations (2.25) and (A.10). Further support stems from the description of the eMZVs' constant terms through the Drinfeld associator [30-32] in equation (2.43) below.

Usual MZVs show up in many relations between eMZVs such as equation (2.36). While crucial for matching the constant term for the eMZVs in question, we will not count them as indecomposable eMZVs. Instead, they will arise as suitably chosen boundary conditions for a differential equation to be elaborated upon below.

Table 1 shows a possible (non-canonical) choice of indecomposable eMZVs for weights up to 14 and length up to and including five. The need for higher-length Fay relations increases the computational complexity in the classification of indecomposable eMZVs using the above procedure. Hence, comparing the $\tau$-dependence will enter as an additional method in the next subsection to extend the results in the table to higher lengths and weights. Still, shuffle, reflection and Fay relations were assembled completely at $\ell_{\omega}=2$, at $\ell_{\omega}=3$ with $w_{\omega} \leqslant 14$, at $\ell_{\omega}=4$ with $w_{\omega} \leqslant 9$ as well as at $\ell_{\omega}=5$ with $w_{\omega} \leqslant 6$, and additional eMZV relations at those weights and lengths have been ruled out on the basis of their $q$-expansion. Continuing the search for indecomposable eMZVs as described in previous and subsequent subsections leads to table 2, in which the number of indecomposable eMZVs for a certain length and weight are noted. Basis rules for rewriting each eMZV in terms of those indecomposable elements can be obtained in digital form from the web page https://tools. aei.mpg.de/emzv and are available up to and including weights 30, 18, 12, 10 for lengths $3,4,5,6$, respectively.

\subsection{Constant term and q-expansion}

The system of relations discussed in the previous section did not require any information on the eMZVs' functional dependence on the modular parameter $\tau$. In this section, we determine the Fourier expansion in $q=\mathrm{e}^{2 \pi \mathrm{i} \tau}$ based on a first-order differential equation in $\tau$ along with a boundary value at $\tau \rightarrow \mathrm{i} \infty$. This will not only provide crosschecks for the above eMZVrelations but will also lead to the more efficient approach to classifying indecomposable eMZVs at higher length and weight in section 4.

2.3.1. Constant term. The constant term of an eMZV can be determined explicitly using results of [1]. By construction, the elliptic KZB associator $A(\tau)$ is the generating series of eMZVs

$\mathrm{e}^{\pi \mathrm{i}[y, x]} A(\tau) \equiv \sum_{r \geqslant 0}(-1)^{r} \sum_{n_{1}, n_{2}, \ldots, n_{r} \geqslant 0} \omega\left(n_{1}, n_{2}, \ldots, n_{r}\right) a d_{x}^{n_{r}}(y) \ldots a d_{x}^{n_{2}}(y) a d_{x}^{n_{1}}(y)$,

and captures the monodromy of the elliptic KZB equation [22, 23] along the path [0, 1]. The prefactor $\mathrm{e}^{\pi \mathrm{i}[y, x]}$ is adjusted to the regularization scheme in equation (2.16). The variables $x$ and $y$ generate a complete, free algebra $\mathbb{C}\langle\langle x, y\rangle\rangle$ of formal power series with complex coefficients, whose multiplication is the concatenation product, and the convention for the adjoint action is 
Table 1. A possible choice of indecomposable eMZVs up to weight 14 and length 5. A table containing the elements missing here is available at https://tools.aei.mpg. de/emzv.

\begin{tabular}{|c|c|c|c|c|c|c|c|c|}
\hline \multicolumn{4}{|c|}{$\ell_{\omega}$} & \multicolumn{5}{|c|}{$\ell_{\omega}$} \\
\hline$w_{\omega}$ & 2 & 3 & 4 & $w_{\omega}$ & 2 & 3 & 4 & 5 \\
\hline 1 & $\omega(0,1)$ & & $\omega(0,0,1,0)$ & 2 & & $\omega(0,0,2)$ & & $\omega(0,0,0,0,2)$ \\
\hline 3 & $\omega(0,3)$ & & $\omega(0,0,0,3)$ & 4 & & $\omega(0,0,4)$ & & $\omega(0,0,0,0,4)$ \\
\hline \multirow[t]{2}{*}{5} & $\omega(0,5)$ & & $\omega(0,0,0,5)$ & & & & & $\omega(0,0,0,1,3)$ \\
\hline & & & $\omega(0,0,2,3)$ & 6 & & $\omega(0,0,6)$ & & $\omega(0,0,0,0,6)$ \\
\hline \multirow[t]{3}{*}{7} & $\omega(0,7)$ & & $\omega(0,0,0,7)$ & & & & & $\omega(0,0,0,1,5)$ \\
\hline & & & $\omega(0,0,2,5)$ & & & & & $\omega(0,0,0,2,4)$ \\
\hline & & & $\omega(0,0,4,3)$ & & & & & $\omega(0,0,2,2,2)$ \\
\hline \multirow[t]{4}{*}{9} & $\omega(0,9)$ & & $\omega(0,0,0,9)$ & 8 & & $\omega(0,0,8)$ & & $\omega(0,0,0,0,8)$ \\
\hline & & & $\omega(0,0,2,7)$ & & & $\omega(0,3,5)$ & & $\omega(0,0,0,1,7)$ \\
\hline & & & $\omega(0,0,4,5)$ & & & & & $\omega(0,0,0,2,6)$ \\
\hline & & & $\omega(0,1,3,5)$ & & & & & $\omega(0,0,1,2,5)$ \\
\hline \multirow[t]{5}{*}{11} & $\omega(0,11)$ & & $\omega(0,0,0,11)$ & & & & & $\omega(0,0,2,2,4)$ \\
\hline & & & $\omega(0,0,2,9)$ & 10 & & $\omega(0,0,10)$ & & $\omega(0,0,0,0,10)$ \\
\hline & & & $\omega(0,0,4,7)$ & & & $\omega(0,3,7)$ & & $\omega(0,0,0,1,9)$ \\
\hline & & & $\omega(0,1,3,7)$ & & & & & and 7 more \\
\hline & & & $\omega(0,3,3,5)$ & 12 & & $\omega(0,0,12)$ & & $\omega(0,0,0,0,12)$ \\
\hline \multirow[t]{7}{*}{13} & $\omega(0,13)$ & & $\omega(0,0,0,13)$ & & & $\omega(0,3,9)$ & & $\omega(0,0,0,1,11)$ \\
\hline & & & $\omega(0,0,2,11)$ & & & & & $\omega(0,0,0,2,12)$ \\
\hline & & & $\omega(0,0,4,9)$ & & & & & and 11 more \\
\hline & & & $\omega(0,1,3,9)$ & 14 & & $\omega(0,0,14)$ & & $\omega(0,0,0,0,14)$ \\
\hline & & & $\omega(0,1,5,7)$ & & & $\omega(0,3,11)$ & & $\omega(0,0,0,1,13)$ \\
\hline & & & $\omega(0,3,3,7)$ & & & $\omega(0,5,9)$ & & $\omega(0,0,0,2,12)$ \\
\hline & & & $\omega(0,3,5,5)$ & & & & & and many more \\
\hline
\end{tabular}

$$
\operatorname{ad}_{x}(y) \equiv[x, y], \quad a d_{x}^{n}(y)=\underbrace{[x, \ldots[x,[x, y]] \ldots]}_{n \text { times }} .
$$

Note that the appearance of eMZVs in equation (2.37) along with non-commutative words in $x$ and $y$ allows for an alternative enumeration scheme using a two-letter alphabet, see section 2.1 .

Enriquez proved that $A(\tau)$ admits the asymptotic expansion as $\tau \rightarrow \mathrm{i} \infty[1]$

$$
A(\tau)=\Phi(\tilde{y}, t) \mathrm{e}^{2 \pi \mathrm{i} \tilde{y}} \Phi(\tilde{y}, t)^{-1}+\mathcal{O}\left(\mathrm{e}^{2 \pi \mathrm{i} \tau}\right),
$$

where $\mathcal{O}\left(\mathrm{e}^{2 \pi \mathrm{i} \tau}\right)$ refers to the non-constant terms in equation (2.15) exclusively. In the above equation, the genus-one alphabet consisting of $x, y$ is translated into a genus-zero alphabet involving

$$
t \equiv[y, x], \quad \tilde{y} \equiv-\frac{a d_{x}}{\mathrm{e}^{2 \pi \mathrm{i} a d_{x}}-1}(y),
$$

and $\Phi$ denotes the Drinfeld associator [30-32]

$$
\Phi\left(e_{0}, e_{1}\right) \equiv \sum_{\hat{W} \in\left\langle e_{0}, e_{1}\right\rangle} \zeta^{\omega}(W) \cdot \hat{W} .
$$




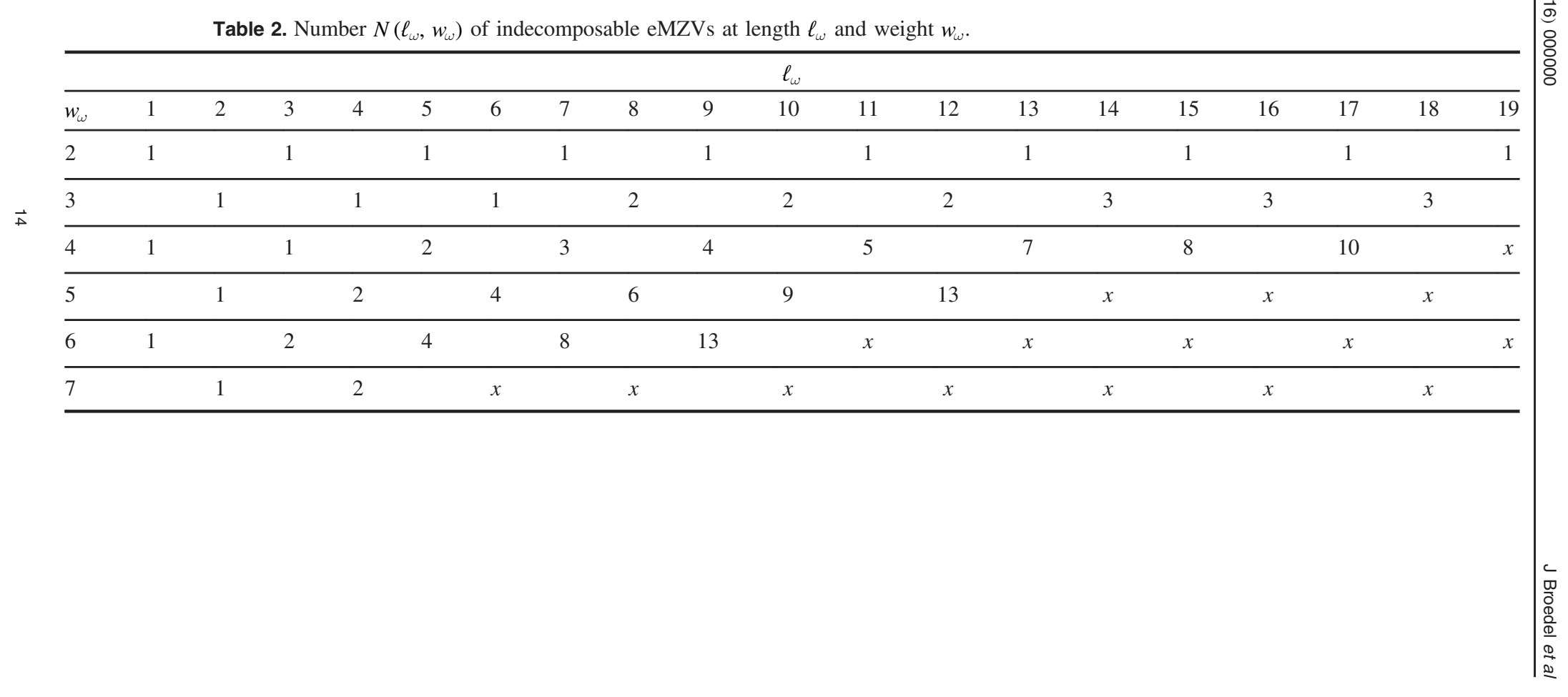


The sum over $\hat{W} \in\left\langle e_{0}, e_{1}\right\rangle$ includes all non-commutative words in letters $e_{0}$ and $e_{1}$, and the word $W$ is obtained from $\hat{W}$ by replacing letters $e_{0}$ and $e_{1}$ by 0 and 1 , respectively. Then, $\zeta^{\omega}(W)$ denote shuffle-regularized MZVs [33] which are uniquely determined from equation (3.1), the shuffle product equation (3.2) and the definition $\zeta^{\omega}(0)=\zeta^{\omega}(1)=0$ for words of length one. Consequently, the first few terms of $\Phi\left(e_{0}, e_{1}\right)$ are given by

$$
\Phi\left(e_{0}, e_{1}\right)=1-\zeta_{2}\left[e_{0}, e_{1}\right]-\zeta_{3}\left[e_{0}+e_{1},\left[e_{0}, e_{1}\right]\right]+\ldots
$$

From equations (2.37) and (2.39), the generating series of constant terms $\omega_{0}\left(n_{1}, \ldots, n_{r}\right)$ of eMZVs is immediately obtained as

$\sum_{r \geqslant 0}(-1)^{r} \sum_{n_{1}, \ldots, n_{r} \geqslant 0} \omega_{0}\left(n_{1}, \ldots, n_{r}\right) a d_{x}^{n_{r}}(y) \ldots a d_{x}^{n_{1}}(y)=\mathrm{e}^{\pi \mathrm{i}[y, x]} \Phi(\tilde{y}, t) \mathrm{e}^{2 \pi \mathrm{i} \tilde{y}} \Phi(\tilde{y}, t)^{-1}$.

In order to transfer information from the right-hand side of equation (2.43) to the constant terms of eMZVs on the left-hand side, it remains to expand words in the alphabet $\{\tilde{y}, t\}$ in equation (2.40) as formal series of words in the alphabet $\left\{a d_{x}^{n}(y) \mid n \geqslant 0\right\}$ and then to compare the coefficients of both sides.

Perhaps surprisingly, the case where all $n_{i} \neq 1$ is very simple to treat. In that case, only the middle term $\mathrm{e}^{2 \pi \mathrm{i} \tilde{y}}$ from equation (2.43) yields a non-trivial contribution, and therefore we have

$$
\left.\omega_{0}\left(n_{1}, n_{2}, \ldots, n_{r}\right)\right|_{n_{i} \neq 1}=\left\{\begin{array}{ll}
0 & \text { if at least one } n_{i} \text { is odd, and all } n_{i} \neq 1 \\
\frac{1}{r !} \prod_{i=1}^{r}\left(-2 \zeta_{n_{i}}\right) & \text { if all } n_{i} \text { are even }
\end{array} .\right.
$$

In particular, one finds

$$
\omega(\underbrace{0,0, \ldots, 0}_{n \text { times }})=\frac{1}{n !},
$$

which is perfectly in line with $f^{(0)} \equiv 1$. On the other hand, in presence of $n_{i}=1$ at some places, a general formula for the constant term is very cumbersome. Simple instances include

$$
\begin{gathered}
\omega_{0}(1,0)=-\frac{\mathrm{i} \pi}{2}, \quad \omega_{0}(1,0,0)=-\frac{\mathrm{i} \pi}{4}, \quad \omega_{0}(1,0,0,0)=-\frac{\mathrm{i} \pi}{12}-\frac{\zeta_{3}}{24 \zeta_{2}} \\
\omega_{0}(0,1,1,0,0)=\frac{\zeta_{2}}{15}, \quad \omega_{0}(1,0,1,1,0,0)=-\frac{\mathrm{i} \pi \zeta_{2}}{30}-\frac{\zeta_{3}}{8}-\frac{17 \zeta_{5}}{96 \zeta_{2}}
\end{gathered}
$$

with generalizations in equation (B.4). Replacing $n_{i}=0$ in the above identities by even values $n_{i}=2 k$ amounts to multiplication with $-2 \zeta_{2 k}$ on the right-hand side.

2.3.2. q-expansion. The $q$-dependent terms in the expansion can be determined using the known form of the $\tau$-derivative of eMZVs. In théorème 3.3 of [1], the derivative of a generating functional for eMZVs is presented, which translates as follows into derivatives of individual eMZVs in our conventions: 


$$
\begin{aligned}
2 \pi \mathrm{i} & \frac{\mathrm{d}}{\mathrm{d} \tau} \omega\left(n_{1}, \ldots, n_{r}\right)=-4 \pi^{2} q \frac{\mathrm{d}}{\mathrm{d} q} \omega\left(n_{1}, \ldots, n_{r}\right) \\
= & n_{1} G_{n_{1}+1} \omega\left(n_{2}, \ldots, n_{r}\right)-n_{r} G_{n_{r}+1} \omega\left(n_{1}, \ldots, n_{r-1}\right) \\
& +\sum_{i=2}^{r}\left\{(-1)^{n_{i}}\left(n_{i-1}+n_{i}\right) G_{n_{i-1}+n_{i}+1} \omega\left(n_{1}, \ldots, n_{i-2}, 0, n_{i+1}, \ldots, n_{r}\right)\right. \\
& \quad-\sum_{k=0}^{n_{i-1}+1}\left(n_{i-1}-k\right)\left(\begin{array}{c}
n_{i}+k-1 \\
k
\end{array}\right) G_{n_{i-1}-k+1} \omega\left(n_{1}, \ldots, n_{i-2}, k+n_{i}, n_{i+1}, \ldots, n_{r}\right) \\
& \left.\quad+\sum_{k=0}^{n_{i}+1}\left(n_{i}-k\right)\left(\begin{array}{c}
n_{i-1}+k-1 \\
k
\end{array}\right) G_{n_{i}-k+1} \omega\left(n_{1}, \ldots, n_{i-2}, k+n_{i-1}, n_{i+1}, \ldots, n_{r}\right)\right\} .
\end{aligned}
$$

The Eisenstein series $G_{k} \equiv G_{k}(\tau)$ on the right-hand side are defined by ${ }^{7}$

$$
\begin{aligned}
& G_{0}(\tau) \equiv-1 \\
& G_{k}(\tau) \equiv \begin{cases}\sum_{\substack{m, n \in \mathbb{Z} \\
(m, n) \neq(0,0) \\
0}} \frac{1}{(m+n \tau)^{k}} & : k>0 \text { even, }\end{cases} \\
& \hline k>0 \text { odd. }
\end{aligned}
$$

Positive even values of $k$ admit a series expansion in the modular parameter:

$$
G_{k}(\tau)=2 \zeta_{k}+\frac{2(-1)^{k / 2}(2 \pi)^{k}}{(k-1) !} \sum_{m, n=1}^{\infty} m^{k-1} q^{m n} \quad k>0 \text { even. }
$$

Using the above formulæ and the known expansion of the Eisenstein series $G_{k}$ in equation (2.49), one can recursively obtain the explicit $q$-expansion for any eMZV: The length of eMZVs on the right-hand side of equation (2.47) is decreased by one compared to the left-hand side, and the recursion terminates with the constant eMZVs at length one given by equation (2.17).

In addition, one finds from equation (2.47) that only divergent eMZVs with $n_{1}=1$ or $n_{r}=1$ lead to the non-modular $G_{2}$, see the discussion around equation (2.16). In all other situations which lead to the non-modular $G_{2}$ in the last three lines, the respective terms cancel out. Not surprisingly, the interesting and boring character of eMZVs is preserved by equation (2.47): the decreased length on the right-hand side is compensated by an increased weight.

Also, note that the differential equation (2.47) contains no MZV terms. In fact, the only way through which MZVs enter the stage of eMZVs is by means of the constant term equation (2.39) of the KZB associator. As mentioned earlier, this constant term can be thought of as a boundary-value prescription for the differential equation (2.47), thereby determining eMZVs uniquely.

2.3.3. eMZV relations from the q-expansion. Based on the $q$-expansions described above, relations between eMZVs can be checked and ruled out by comparing their Fourier representations. In practice, one writes down an ansatz comprised from interesting eMZVs

7 The case $k=2$ requires the Eisenstein summation prescription

$$
\sum_{m, n \in \mathbb{Z}} a_{m, n} \equiv \lim _{N \rightarrow \infty M \rightarrow \infty} \lim _{n=-N m=-M}^{N} \sum_{m, n}^{M} a_{m}
$$


and products thereof with uniform weight and an upper bound on the length of interest, each term supplemented with fudge coefficients.

Naïvely, one could calculate the $q$-expansions of all constituents up to a certain order $q^{N_{\max }}$ and impose a matching along with each Fourier mode $q^{n}$ for $0 \leqslant n \leqslant N_{\max }$. This allows to fix the above fudge coefficients and to check the relations' validity up to-in principlearbitrary order. In an early stage of the project, our computer implementation of this approach with $N_{\max }=160$ was far more efficient compared to the analysis of reflection, shuffle and Fay identities and lead to substantial parts of the data shown in tables 1 and 2.

However, since the comparison of $q$-expansions has to be cut off at some chosen power of $q$, a proof for relations using the method is impossible by construction. Even worse, this naïve method fails to capture the structural insight from equation (2.47) that any $q$ dependence in eMZVs stems from iterated integrals of Eisenstein series. This crucial property is exploited in section 4 , confirming the entries of table 2 in a rigorous and conceptually by far more elegant manner.

While the naïve comparison of Fourier coefficients merely provides a lower bound for the number of indecomposable eMZVs for a given weight and length, the description of eMZVs in terms of iterated Eisenstein integrals in section 4 yields complementary upper bounds. Under the additional assumption that different iterated Eisenstein integrals are linearly independent, these upper bounds are indeed saturated. However, since we do not attempt to prove their linear independence, the naïve matching of Fourier coefficients closes the associated loophole at the weights and lengths under consideration.

\section{MZVs and the $\phi$-map}

In this section we gather information on the structure of MZVs, which are to be compared with those found for eMZVs in section 4 below. While represented as nested sums in equation (2.1) in section 2, they can alternatively be defined as iterated integrals

$$
\begin{aligned}
\zeta_{n_{1}, n_{2}, \ldots, n_{r}} & =\int_{0 \leqslant z_{i} \leqslant z_{i+1} \leqslant 1} \omega_{1} \underbrace{\omega_{0} \ldots \omega_{0}}_{n_{1}-1} \omega_{1} \underbrace{\omega_{0} \ldots \omega_{0}}_{n_{2}-1} \ldots \omega_{1} \underbrace{\omega_{0} \ldots \omega_{0}}_{n_{r}-1} \\
& =\zeta(\underbrace{0 \ldots 0}_{n_{1}-1} 1 \underbrace{0 \ldots 0}_{n_{2}-1} \ldots 1 \underbrace{0 \ldots 0}_{n_{r}-1})
\end{aligned}
$$

over the differential forms $\omega_{0} \equiv \frac{\mathrm{d} z}{z}$ and $\omega_{1} \equiv \frac{\mathrm{d} z}{1-z}$ with all $z_{i}$ on the real line. The MZV $\zeta_{n_{1}, \ldots, n_{r}}$ is said to have weight $w=\sum_{i=1}^{r} n_{i}$ and depth $r$. Written in terms of words $W$ composed from the letters 0 and 1 , which correspond to the differential forms $\omega_{0}$ and $\omega_{1}$ in equation (3.1), respectively, $\zeta$ 's satisfy the shuffle product:

$$
\zeta\left(W_{1}\right) \zeta\left(W_{2}\right)=\zeta\left(W_{1} w W_{2}\right) .
$$

There is also a second product structure on MZVs, the stuffle product. Its simplest instance reads

$$
\zeta_{m} \zeta_{n}=\zeta_{m, n}+\zeta_{n, m}+\zeta_{m+n}
$$

It follows from either equations (3.2) or (3.3) that the $\mathbb{Q}$-span $\mathcal{Z}$ of all MZVs is a subalgebra of $\mathbb{R}$. Conjecturally, $\mathcal{Z}$ is graded by the weight of the MZVs

$$
\mathcal{Z}=\underset{w=0}{\oplus} \mathcal{Z}_{w}
$$


Table 3. A possible choice for the basis elements of $\mathcal{Z}_{w}$ for $2 \leqslant w \leqslant 12$.

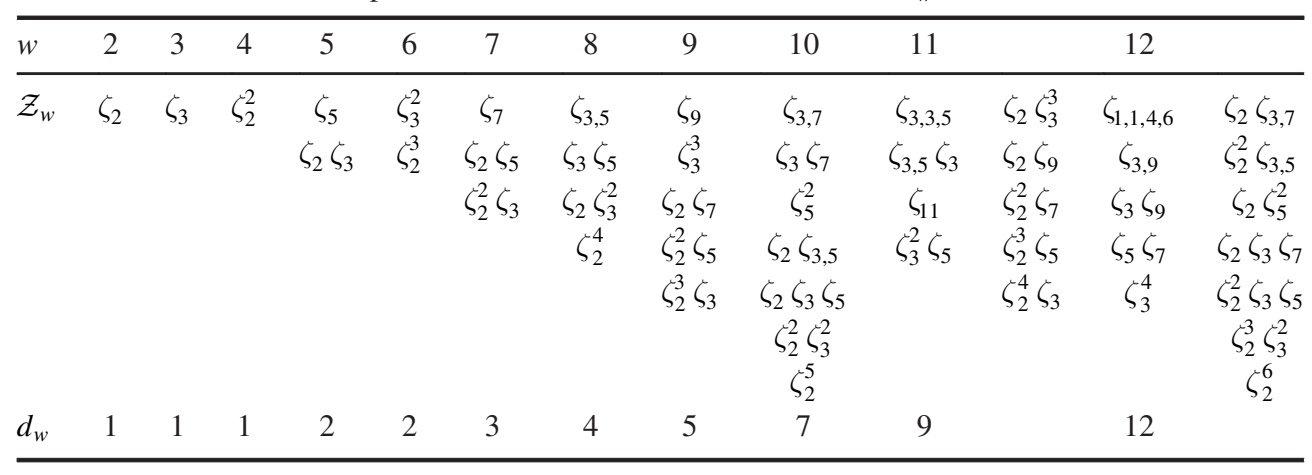

where the dimensions $d_{w}$ of $\mathcal{Z}_{w}$ have been conjectured to be $d_{w}=d_{w-2}+d_{w-3}$ where $d_{0}=1, d_{1}=0$ and $d_{2}=1$ [14]. A possible choice of basis elements for each weight $w$ is given in table 3 , for higher weights consult [15].

Single $\zeta$-functions of even weight are rather different from their odd-weight counterparts: all single zeta values of even weight $2 n$ can be expressed as rational multiples of $\pi^{2 n}$, which renders them transcendental numbers immediately. For odd single zeta values, however, there is no analogous property: there are no known relations relating two single zeta values of distinct odd weight, and in fact no such relations are expected. Also, although expected, none of the odd $\zeta$-values has been proven to be transcendental so far: the only known facts are the irrationality of $\zeta_{3}$ as well as the existence of an infinite number of odd irrational $\zeta$ 's [34, 35].

\subsection{Hopf algebra structure of MZVs}

The basis elements in table 3 have been chosen by convenience preferring short and simple $\zeta$ 's. However, the choice of basis elements does not seem to be intuitive at all, as is exemplified by the appearance of $\zeta_{1,1,4,6}$ at weight 12 . It would be desirable to find a language in which one can write down a basis for MZVs in a more transparent way, with all relations built in once the translation is performed. This language does indeed exist: it is furnished by the graded Hopf algebra comodule $\mathcal{U}$, which is composed from words

$$
f_{2 i_{1}+1} \ldots f_{2 i_{r}+1} f_{2}^{k}, \quad \text { with } r, k \geqslant 0 \text { and } i_{1}, \ldots, i_{r} \geqslant 1
$$

of weight $w=2\left(i_{1}+\ldots+i_{r}\right)+r+2 k$. While words in the letters $f_{2 i+1}$ span a Hopf algebra endowed with a commutative shuffle product, the Hopf algebra comodule $\mathcal{U}$ is obtained upon adjoining powers of $f_{2}$, which commute with all $f_{2 i+1}$ [12]. Writing down all words of the form in equation (3.5), one indeed finds the dimension of $\mathcal{U}_{w}$ to match the expected dimension $d_{w}$ of $\mathcal{Z}_{w}$, which is a first indicator that the Hopf algebra comodule $\mathcal{U}$ does indeed shed light on the algebraic structure of MZVs.

In a next step MZVs need to be related to elements in $\mathcal{U}$. Unfortunately, due to the difficult problem of excluding algebraic relations between MZVs, this cannot be done directly. In order to circumvent this issue, one lifts $\mathrm{MZVs} \zeta$ to so-called motivic MZVs $\zeta^{\mathfrak{m}}$, which have a more elaborate definition $[12,36,37]$, but which still satisfy the same shuffle and stuffle product formulæ as the MZVs equations (3.2) and (3.3). Moreover, passing from MZVs to motivic MZVs has the advantage that many of the desirable, but currently unproven facts about MZVs are in fact proven for motivic MZVs. In particular, the commutative algebra $\mathcal{H}$ of motivic MZVs is by definition graded for the weight, and carries a well-defined 
motivic coaction, first written down by Goncharov [36] and further studied by Brown $[11,12,37]$.

With the availability of $\mathcal{H}$ the only remaining piece is the construction of an isomorphism $\phi$ of graded algebra comodules

$$
\phi: \mathcal{H} \rightarrow \mathcal{U}
$$

whose existence is guaranteed by the main result of [12]. The map $\phi$, which assigns to each motivic MZV a linear combination of the words defined in equation (3.5), is thoroughly described and explored in [11]. As pointed out in the reference, the map $\phi$ is non-canonical and depends on the choice of an algebra basis of $\mathcal{H}$. The requirement that all odd single motivic MZVs as well as $\zeta_{2}$ should be contained in this basis leads to

$$
\phi\left(\zeta_{k}^{\mathfrak{m}}\right)=f_{k}, \quad k=2,3,5,7, \ldots
$$

Unfortunately, this convention does not determine $\phi$ uniquely, since not every motivic MZV can be expressed in terms of motivic single zeta values only. However, as pointed out in [11], the map $\phi$ preserves all relations between motivic MZVs for any choice of algebra basis of $\mathcal{H}$, for example (see equation (3.3)):

$$
\phi\left(\zeta_{m}^{\mathfrak{m}} \zeta_{n}^{\mathfrak{m}}\right)=\phi\left(\zeta_{m, n}^{\mathfrak{m}}\right)+\phi\left(\zeta_{n, m}^{\mathfrak{m}}\right)+\phi\left(\zeta_{m+n}^{\mathfrak{m}}\right) .
$$

In order to give explicit examples of the decomposition of motivic MZVs into the $f$-alphabet, let us choose the following algebra basis up to weight 12, upon which table 3 is modeled implicitly

$$
\left\{\zeta_{2}^{\mathfrak{m}}, \zeta_{3}^{\mathfrak{m}}, \zeta_{5}^{\mathfrak{m}}, \zeta_{7}^{\mathfrak{m}}, \zeta_{3,5}^{\mathfrak{m}}, \zeta_{9}^{\mathfrak{m}}, \zeta_{3,7}^{\mathfrak{m}}, \zeta_{11}^{\mathfrak{m}}, \zeta_{3,3,5}^{\mathfrak{m}}, \zeta_{1,1,4,6}^{\mathfrak{m}}, \zeta_{3,9}^{\mathfrak{m}}\right\}
$$

With this choice of basis, one finds for example

$$
\begin{aligned}
& \phi\left(\zeta_{3,9}^{\mathfrak{m}}\right)=-6 f_{5} f_{7}-15 f_{7} f_{5}-27 f_{9} f_{3} \\
& \phi\left(\zeta_{3,3,5}^{\mathfrak{m}}\right)=-5 f_{5} f_{3} f_{3}+\frac{4}{7} f_{5} f_{2}^{3}-\frac{6}{5} f_{7} f_{2}^{2}-45 f_{9} f_{2} .
\end{aligned}
$$

The application of the $\phi$-map in the context of the low-energy expansion of superstring treelevel amplitudes as well as several higher-weight examples can be found in [38].

\section{Indecomposable eMZVs, Eisenstein series and the derivation algebra}

As described in section 2, indecomposable eMZVs at a certain weight and length can be in principle inferred from considering reflection, shuffle and Fay relations. For higher weights and lengths, however, it is favorable to employ a computer implementation based on comparing $q$-expansions of eMZVs which in turn can be obtained recursively from equation (2.47). In this section we are going to provide an algorithm which does not only deliver the appropriate indecomposable elements as listed in table 1 but as well explains their number at a given length and weight.

As described in the previous section, the appropriate mathematical idea for standard motivic MZVs is to map them to the non-commutative words composed from letters $f_{w}$ in equation (3.5) using the map $\phi$. For the elliptic case we will construct an isomorphism $\psi$ relating the $\omega$-representation of eMZVs to non-commutative words composed from letters $g_{w}$, which in turn arise as labels of iterated Eisenstein integrals $\gamma$ to be defined below. 


\subsection{Iterated Eisenstein integrals}

Given that the $q$-expansion of eMZVs can be iteratively generated from the Eisenstein series $G_{k}$ employing equation (2.47), we will now describe eMZVs based on combinations of $G_{k}$. Instead of representing eMZVs as elliptic iterated integrals as in section 2, we will write them as iterated integrals over Eisenstein series $G_{k}$ where the iterated integration is now performed over the modular parameter $\tau$ (or equivalently $q$ ).

Iterated integrals over Eisenstein series arise as a subclass of iterated integrals of modular forms, which have been studied in $[17,18]$. In this section, we will briefly review some of the key definitions in order to embed the subsequent presentation of eMZVs into a broader context.

Iterated integrals of modular forms or iterated Shimura integrals $[17,18]$ are defined via

$$
\begin{aligned}
& \int_{i \infty>\tau_{1}>\tau_{2}>\cdots>\tau} \mathrm{d} \tau_{1}\left(X_{1}-\tau_{1} Y_{1}\right)^{k_{1}-2} \mathcal{F}_{k_{1}}\left(\tau_{1}\right) \mathrm{d} \tau_{2}\left(X_{2}-\tau_{2} Y_{2}\right)^{k_{2}-2} \mathcal{F}_{k_{2}}\left(\tau_{2}\right) \cdots \\
& \cdots \mathrm{d} \tau_{n}\left(X_{n}-\tau_{n} Y_{n}\right)^{k_{n}-2} \mathcal{F}_{k_{n}}\left(\tau_{n}\right),
\end{aligned}
$$

where $\mathcal{F}_{k}(\tau)$ is a modular form of weight $k$ and the modular group acts on commutative variables $X_{i}$ and $Y_{i}$ as to render equation (4.1) modular invariant. The divergences in these integrals caused by the constant terms in the $q$-expansion of the modular forms can be regularized in a manner described in [18]. The key idea of this regularization procedure is to separate the constant part from the remaining $q$-series for each $\mathcal{F}_{k_{j}}(q)$ and to associate a different integration prescription to it. The mathematical justification of this procedure is furnished by the theory of tangential base points [39]. In the present case, one regularizes the integral with respect to the tangential base point $\overrightarrow{1}_{\infty}$ [18].

In the context of eMZVs in equation (2.11), we encounter special cases of the iterated Shimura integrals defined above, evaluated at $X_{i}=1$ and $Y_{i}=0$. Furthermore, the $\tau$-derivative of eMZVs in equation (2.47) involves no modular forms $\mathcal{F}_{k}$ other than Eisenstein series $G_{k}$. This motivates to study the following iterated Eisenstein integrals as building blocks for eMZVs

$$
\begin{aligned}
& \gamma\left(k_{1}, k_{2}, \ldots, k_{n} ; q\right) \equiv \frac{1}{4 \pi^{2}} \int_{0 \leqslant q^{\prime} \leqslant q} \operatorname{dlog} q^{\prime} \gamma\left(k_{1}, \ldots, k_{n-1} ; q^{\prime}\right) G_{k_{n}}\left(q^{\prime}\right) \\
& =\frac{1}{\left(4 \pi^{2}\right)^{n}} \int_{0 \leqslant q_{i}<q_{i+1} \leqslant q} \operatorname{dlog} q_{1} G_{k_{1}}\left(q_{1}\right) \operatorname{dlog} q_{2} G_{k_{2}}\left(q_{2}\right) \ldots \operatorname{dlog} q_{n} G_{k_{n}}\left(q_{n}\right),
\end{aligned}
$$

where the number $n$ of integrations will be referred to as the length $\ell_{\gamma}$, and the weight is given by $w_{\gamma}=\sum_{i=1}^{n} k_{i}$. The definition in equation (4.2) as an iterated integral immediately implies

$$
\begin{gathered}
\frac{\mathrm{d}}{\operatorname{d} \log q} \gamma\left(k_{1}, k_{2}, \ldots, k_{n} ; q\right)=\frac{G_{k_{n}}(q)}{4 \pi^{2}} \gamma\left(k_{1}, k_{2}, \ldots, k_{n-1} ; q\right) \\
\gamma\left(n_{1}, n_{2}, \ldots, n_{r} ; q\right) \gamma\left(k_{1}, k_{2}, \ldots, k_{s} ; q\right)=\gamma\left(\left(n_{1}, n_{2}, \ldots, n_{r}\right) \omega\left(k_{1}, k_{2}, \ldots, k_{s}\right) ; q\right),
\end{gathered}
$$

where the dependence on $q$ will be suppressed in most cases: $\gamma(\ldots) \equiv \gamma(\ldots ; q)$. The integrals in equation (4.2) generally diverge due to the constant term in $G_{k_{1}}=2 \zeta_{k_{1}}+\mathcal{O}(q)$ and can be regularized using the procedure discussed around equation (4.7) while preserving equations (4.3) and (4.4).

As will be explained in detail below, eMZVs can be expressed in terms of particular linear combinations of iterated Eisenstein integrals in equation (4.2) such that all possible divergences cancel. An alternative description of eMZVs which manifests the absence of divergences and admits convenient formulæ for their $q$-expansion will be given in section 4.6. 


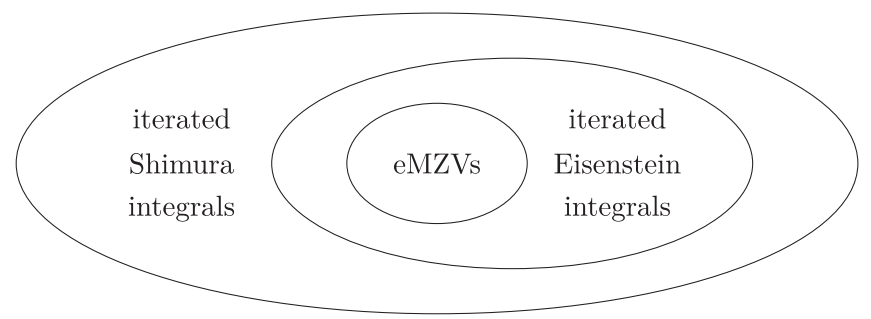

Figure 1. Relation between different type of iterated integrals discussed.

The convergent linear combinations of equation (4.2) occurring in eMZVs will turn out to be governed by a special derivation algebra $\mathfrak{u}$. The situation is summarized in figure 1: eMZVs are a special case of iterated Eisenstein integrals equation (4.2) which in turn span a subspace of iterated Shimura integrals equation (4.1).

4.1.1. Regularization. Even though eMZVs can be assembled from convergent iterated integrals over modular parameters-see section 4.6-we shall sketch a regularization procedure for the iterated Eisenstein integrals in equation (4.2) to render individual terms in the subsequent description of eMZVs well-defined. Let us consider the simplest case, namely that of an iterated integral of length one:

$$
\gamma(k)=\frac{1}{4 \pi^{2}} \int_{0}^{q} G_{k}\left(q^{\prime}\right) \operatorname{dlog} q^{\prime} .
$$

The term $\left(G_{k}\left(q^{\prime}\right)-2 \zeta_{k}\right)$ dlog $q^{\prime}$ is straightforward to integrate from 0 to $q$, since it has no poles on the integration domain $0 \leqslant q^{\prime} \leqslant q$. On the other hand, integration of the term $2 \zeta_{k}$ d $\log q^{\prime}$ in isolation requires regularization, due to the presence of a simple pole at $q^{\prime}=0$. The regularization scheme employed in this case, however, is entirely analogous to the regularization scheme for multiple polylogarithms, MZVs or eMZVs: One introduces a small parameter $\varepsilon>0$, then expands the integral

$$
2 \zeta_{k} \int_{\varepsilon}^{q} \operatorname{d} \log q^{\prime}=2 \zeta_{k}(\log q-\log \varepsilon)
$$

as a polynomial in $\log (\varepsilon)$ and finally takes the constant term in this expansion. Using this procedure in the length one case, one obtains from equation (4.5)

$$
\gamma(k)=\frac{1}{4 \pi^{2}} \int_{0}^{q} G_{k}\left(q^{\prime}\right) \operatorname{dlog} q^{\prime}=\frac{1}{4 \pi^{2}}\left(2 \zeta_{k} \log q+\frac{2(-1)^{k / 2}(2 \pi)^{k}}{(k-1) !} \sum_{m, n=1}^{\infty} \frac{m^{k-2}}{n} q^{m n}\right) .
$$

The regularization procedure for a general iterated Eisenstein integral $\gamma\left(k_{1}, k_{2}, \ldots, k_{n}\right)$ as in 4.2 is deduced from the length one case, using the shuffle product formula. Full details can be found in [18].

\section{2. eMZVs as iterated Eisenstein integrals}

As a first example on how to express eMZVs in terms of iterated Eisenstein integrals, let us consider equation (2.47) for two simple types of eMZVs (recalling equation (2.2) and $\left.G_{0} \equiv-1\right)$ : 


$$
\begin{aligned}
& 2 \pi \mathrm{i} \frac{\mathrm{d}}{\mathrm{d} \tau} \omega(0, n)=-4 \pi^{2} q \frac{\mathrm{d}}{\mathrm{d} q} \omega(0, n)=-2 n \zeta_{n+1} G_{0}-n G_{n+1}, \quad n \text { odd } \\
& 2 \pi \mathrm{i} \frac{\mathrm{d}}{\mathrm{d} \tau} \omega(0,0, n)=-4 \pi^{2} q \frac{\mathrm{d}}{\mathrm{d} q} \omega(0,0, n)=n \omega(0, n+1) G_{0}, n \text { even. }
\end{aligned}
$$

Integration over dlog $q$ relates the eMZVs on the left-hand side to iterated Eisenstein integrals of the form equation (4.2), and the absence of constant terms within $\tau$-derivatives guarantees that the integral converges. This insight will actually be the key ingredient to the simplified representation of eMZVs described in section 4.6 below. The rewriting in equations $(4.8 a)$ and $(4.8 b)$ can be generalized for all eMZVs: using the differential equation (2.47) one can represent their derivative as a sum over Eisenstein series $G_{2 k}$,

$$
\frac{\mathrm{d}}{\operatorname{dlog} q} \omega\left(n_{1}, n_{2}, \ldots, n_{r}\right)=\frac{1}{4 \pi^{2}} \sum_{k=0}^{\infty} \xi_{2 k}\left(n_{1}, n_{2}, \ldots, n_{r}\right) G_{2 k},
$$

where the coefficients $\xi_{2 k}\left(n_{1}, \ldots, n_{r}\right)$ are linear combinations of eMZVs of weight $n_{1}+\ldots+n_{r}+1-2 k$ and length $r-1$. An example of this decomposition is spelled out below equation (4.11).

For the eMZVs appearing in the coefficients $\xi_{2 k}\left(n_{1}, \ldots, n_{r}\right)$ of equation (4.9), the procedure can be repeated to successively reduce the length. Finally, any eMZV can be rewritten in terms of the iterated Eisenstein integrals in equation (4.2). Since the right-hand side of equation (4.9) is a $\tau$-derivative and cannot have a constant term in $q$, its integral over $\operatorname{dlog} q$ is convergent and the first entries of the resulting iterated Eisenstein integrals for any eMZV are interlocked as $\gamma(k, \ldots)+2 \zeta_{k} \gamma(0, \ldots)$.

4.2.1. Examples. Let us return to the examples equations (4.8a) and (4.8b). The differential equation (4.3) immediately implies

$$
\omega(0, n)=\delta_{1, n} \frac{\pi \mathrm{i}}{2}+n\left(\gamma(n+1)+2 \zeta_{n+1} \gamma(0)\right), \quad n \text { odd }
$$

$\omega(0,0, n)=-\frac{1}{3} \zeta_{n}-n(n+1)\left(\gamma(n+2,0)+2 \zeta_{n+2} \gamma(0,0)\right), \quad n$ even,

where $\delta_{1, n} \frac{\pi \mathrm{i}}{2}$ and $-\frac{1}{3} \zeta_{n}$ arise as integration constants w.r.t. $\log q$. Even though all the above iterated Eisenstein integrals $\gamma(n+1), \gamma(n+2,0), \gamma(0)$ and $\gamma(0,0)$ individually require regularization-see the discussion around equation (4.7) - any divergence cancels in the linear combinations of schematic form $\gamma(k, \ldots)+2 \zeta_{k} \gamma(0, \ldots)$ in equations (4.10a) and $(4.10 b)$.

The conversion of eMZVs into $\gamma$ 's amounts to recursively applying the differential equation (2.47) and casting it into the form equation (4.9). At each step, an instance of $G_{k}$ is separated until one has reached eMZVs of the form in equations (4.10a) and (4.10b) exclusively. After converting those into $\gamma$ 's, one reverts the direction and successively integrates using equation (4.2), supplementing integration constants from equation (2.43).

Let us demonstrate the conversion into iterated Eisenstein integrals $\gamma$ for $\omega(0,3,5)$. Employing equation (2.47), one finds

$$
4 \pi^{2} \frac{\mathrm{d}}{\operatorname{dlog} q} \omega(0,3,5)=-15 G_{4} \omega(0,5)+42 \omega(0,9)+3 \omega(4,5),
$$


i.e. we have $\xi_{4}(0,3,5)=-15 \omega(0,5)$ and $\xi_{0}(0,3,5)=-42 \omega(0,9)-3 \omega(4,5)$ in the notation of equation (4.9). While $\omega(0,5)$ and $\omega(0,9)$ can be readily converted into $\gamma$ 's using equations $(4.10 a)$ and $(4.10 b)$, we will have to take another derivative ${ }^{8}$ for $\omega(4,5)$ :

$$
\begin{aligned}
4 \pi^{2} \frac{\mathrm{d}}{\mathrm{d} \log q} \omega(4,5) & =9 G_{10} \omega(0)-15 G_{4} \omega(6)+42 \omega(10) \\
& =9 G_{10}+30 \zeta_{6} G_{4}+84 \zeta_{10} G_{0}
\end{aligned}
$$

Performing the integration equation (4.2) then leads to

$$
\omega(4,5)=9 \gamma(10)+30 \zeta_{6} \gamma(4)+84 \zeta_{10} \gamma(0),
$$

which-after plugged into equation (4.11)—yields

$$
\begin{aligned}
4 \pi^{2} \frac{\mathrm{d}}{\mathrm{d} \log q} \omega(0,3,5)= & -75 G_{4}\left(\gamma(6)+2 \zeta_{6} \gamma(0)\right)+405 \gamma(10) \\
& +90 \zeta_{6} \gamma(4)+1008 \zeta_{10} \gamma(0)
\end{aligned}
$$

After a last integration of the type in equation (4.2) one finally obtains

$$
\begin{aligned}
\omega(0,3,5)= & -405 \gamma(10,0)-75 \gamma(6,4)-\zeta_{6}(150 \gamma(0,4)+90 \gamma(4,0)) \\
& -1008 \zeta_{10} \gamma(0,0)
\end{aligned}
$$

which casts the first indecomposable length-three eMZV beyond equation $(4.10 b)$ into the language of iterated Eisenstein integrals and fits into the pattern $\gamma(k, \ldots)+2 \zeta_{k} \gamma(0, \ldots)$ for the first entries. Further examples of expressing eMZVs as iterated Eisenstein integrals are listed in appendix B.2.

4.2.2. Conversion of weight and length. Length and weight are different between the representation of eMZVs in terms of iterated Eisenstein integrals $\gamma$ and the $\omega$-representation. Denoting length and weight for $\gamma$ and $\omega$ by $\left(\ell_{\gamma}, w_{\gamma}\right)$ and $\left(\ell_{\omega}, w_{\omega}\right)$, respectively, one finds straightforwardly

$$
\ell_{\gamma}=\ell_{\omega}-1 \quad \text { and } \quad w_{\gamma}=\ell_{\omega}-1+w_{\omega}=\ell_{\gamma}+w_{\omega}
$$

such that

$\gamma\left(k_{1}, k_{2}, \ldots, k_{n}\right) \leftrightarrow \mathrm{eMZV}$ in $\omega-$ rep. with $\ell_{\omega}=n+1$ and $w_{\omega}=-n+\sum_{j=1}^{n} k_{j}$,

$\omega\left(n_{1}, n_{2}, \ldots, n_{r}\right) \leftrightarrow$ Eisenstein integral with $\ell_{\gamma}=r-1$ and $w_{\gamma}=r-1+\sum_{j=1}^{r} n_{j}$

Those formulæ, however, are valid for the maximal component only: as illustrated e.g. in equation (4.10b), the presentation of eMZVs in terms of iterated Eisenstein integrals involves different lengths $\ell_{\gamma}$ and weights $w_{\gamma}$. Correspondingly, the maximal component is defined to be comprised from all terms in an eMZVs $\gamma$-representation, which are of length $\ell_{\gamma}$ and weight $w_{\omega}$. Below, we will exclude $\gamma$ 's, which can be represented as shuffle products, from the maximal component. Iterated Eisenstein integrals of length $\ell_{\gamma}-2, \ell_{\gamma}-4, \ldots$ as well as any terms in which weight is carried by MZVs do not belong to the maximal component.

${ }^{8}$ Alternatively, one could use equation (2.33), but for illustrational purposes we will perform the recursion explicitly. 
The examples in equations $(4.10 b)$ and (4.15) give rise to maximal components

$$
\begin{aligned}
& \omega(0,0, n)=-n(n+1) \gamma(n+2,0)+\text { non-maximal terms } \\
& \omega(0,3,5)=-405 \gamma(10,0)-75 \gamma(6,4)+\text { non-maximal terms, }
\end{aligned}
$$

which are defined up to shuffle products of lower-length iterated Eisenstein integrals.

Considering equation $(4.17 a)$, one can create $\gamma$ 's corresponding to $\omega$-representations of negative weight. Since weighting functions $f^{(m)}$ are not defined for negative weight, $\gamma\left(k_{1}, k_{2}, \ldots, k_{n}\right)$ with $\sum_{j=1}^{n} k_{j}<n$ are clearly incompatible with the definition of eMZVs in equation (2.11). However, the connection with the derivation algebra $\mathfrak{u}$ in section 4.3 below will assign a meaning to those $\gamma$ 's in the context of relations between eMZVs at length $\ell_{\omega} \geqslant 6$.

4.2.3. Counting of indecomposable eMZVs. What are the advantages of translating eMZVs into iterated Eisenstein integrals? We would like to derive the set of indecomposable eMZVs with given length and weight from purely combinatorial considerations, similar to writing down all non-commutative words of letters $f$ for standard MZVs (see equation (3.5)). In particular, each indecomposable eMZV in table 1 should be related to a particular combination of shuffle-independent $\gamma$ 's. Correspondingly, the counting of indecomposable $\gamma$ 's of appropriate weight and length should be related to the numbers in table 2 .

In order to assess the viability of iterated Eisenstein integrals $\gamma$ for this purpose, it is worthwhile to recall the following observations:

(a) By construction, constant terms are absent in the differential equation (2.47) for eMZVs. This interlocks the first entries of iterated Eisenstein integrals representing eMZVs in rigid combinations of $\gamma(k, \ldots)+2 \zeta_{k} \gamma(0, \ldots)$. Hence, it is sufficient for counting purposes to focus on $\gamma\left(k_{1}, k_{2}, \ldots, k_{r}\right)$ with $k_{1} \neq 0$.

(b) The choice of indecomposable eMZVs in table 1 contains no further divergent representative besides $\omega(0,1)=\gamma(2)+2 \zeta_{2} \gamma(0)+\frac{i \pi}{2}$. For any weight and length considered, divergences in eMZVs are captured by products with $\gamma(2)$ instead of shuffleirreducible integrals of higher length such as $\gamma(2,4)$. We will assume the continuation of this pattern and confine the choice of labels for all other Eisenstein integrals $\gamma$ at length $\ell_{\gamma} \geqslant 2$ to the set $\{0,4,6, \ldots\}$. This will be justified later on by the observation that the element $\epsilon_{2} \in \mathfrak{u}$ corresponding to $\gamma(2)$ is central by equation (4.27).

(c) The shuffle relations equation (4.4) allow to reduce various linear combinations of iterated Eisenstein integrals to lower length, e.g.

$$
\gamma(4,4)=\frac{1}{2} \gamma(4)^{2} \quad \text { and } \quad \gamma(6) \gamma(4)=\gamma(4,6)+\gamma(6,4)
$$

and the bookkeeping of indecomposable eMZVs boils down to classifying shuffleindependent Eisenstein integrals $\gamma$. At length $\ell_{\gamma}=2$ and weight $w_{\gamma}=10$, possible indecomposable elements read $\gamma(10,0)$ and $\gamma(6,4)$, because $\gamma(4,6)$ can be obtained using shuffling of $\gamma$ 's of lower length. Similarly, $\ell_{\gamma}=2$ and $w_{\gamma}=12$ leaves no indecomposable eMZVs beyond $\gamma(12,0)$ and $\gamma(8,4)$. Let us compare the survey of available Eisenstein integrals with the indecomposable eMZVs in table 1. Eisenstein integrals of length one immediately match with the maximal component of indecomposable eMZVs $\omega(0,2 n+1)$ of length two using equation $(4.10 a)$, so the first non-trivial tests occur at length $\ell_{\omega}=3$, i.e. $\ell_{\gamma}=2$. 
Via equation $(4.10 b)$ one finds indeed $\gamma(4,0), \gamma(6,0)$ and $\gamma(8,0)$ to represent the maximal component of $\omega(0,0,2), \omega(0,0,4)$ and $\omega(0,0,6)$, respectively. For $w_{\gamma}=10$, which corresponds to $w_{\omega}=8$, one can write down two distinct indecomposable elements: $\gamma(10,0)$ and $\gamma(6,4)$. This nicely ties in with the appearance of the second indecomposable $\operatorname{eMZV} \omega(0,3,5)$ at $\ell_{\omega}=3$ and $w_{\omega}=8$, see equations (4.18) and (4.19).

Similarly, the aforementioned indecomposable eMZVs $\gamma(12,0)$ and $\gamma(8,4)$ at weight $w_{\gamma}=12$ are in concordance with the $w_{\omega}=10$ entry of table 1 ,

$$
\begin{aligned}
& \omega(0,0,10)=-\frac{\zeta_{10}}{3}-110 \gamma(12,0)-220 \zeta_{12} \gamma(0,0) \\
& \omega(0,3,7)=-294 \gamma(8,4)-1848 \gamma(12,0)+\text { non-maximal terms. }
\end{aligned}
$$

The appearance of the indecomposable eMZVs $\omega(0,3,5)$ and $\omega(0,3,7)$ beyond $\omega(0,0,2 n)$ matches the existence of shuffle-independent Eisenstein integrals $\gamma(6,4)$ and $\gamma(8,4)$ in addition to $\gamma(10,0)$ and $\gamma(12,0)$.

4.2.4. Surprises from weight-twelve eMZVs and beyond. The literal application of the above reasoning to iterated Eisenstein integrals of weight $w_{\gamma}=14$ suggests indecomposable eMZVs

$$
\begin{aligned}
& \omega(0,0,12) \text { from } \gamma(14,0), \quad \omega(0,3,9) \text { from } \gamma(10,4) \\
& \quad \text { and } \omega(0,5,7) \text { from } \gamma(8,6) .
\end{aligned}
$$

This, however, clashes with the findings noted in table 1 : at $\ell_{\omega}=3$ and $w_{\omega}=12$ we find only two indecomposable eMZVs $\omega(0,0,12)$ and $\omega(0,3,9)$, whereas the above counting of appropriate interated Eisenstein integrals would suggest three indecomposable eMZVs. In particular, $\omega(0,5,7)$ can be expressed in terms of the two indecomposable eMZVs as written in equation (2.35).

In order to explain the discrepancy between indecomposable eMZVs and shuffleindependent iterated Eisenstein integrals, let us inspect the first instance at $w_{\gamma}=14, \ell_{\gamma}=2$, which corresponds to $w_{\omega}=12, \ell_{\omega}=3$. The natural candidates for indecomposable eMZVs besides $\omega(0,0,12)$ have the following $\gamma$-representations

$\omega(0,3,9)=-315 \gamma(8,6)-729 \gamma(10,4)-5616 \gamma(14,0)+$ no-maximal terms

$\omega(0,5,7)=-490 \gamma(8,6)-1134 \gamma(10,4)-5642 \gamma(14,0)+$ non-maximal terms,

and the relation equation $(2.35)$ for $\omega(0,5,7)$ leaves only $\omega(0,0,12)$ and $\omega(0,3,9)$ indecomposable. In general, there seem to be non-obvious restrictions to the Eisenstein integrals $\gamma$ appearing in eMZVs, beyond the observations (a), (b) and (c). In table 4, we have noted the deviations from the expected pattern at lengths $\ell_{\omega} \leqslant 5$. Interestingly, the Eisenstein integrals $\gamma(8,6)$ and $\gamma(10,4)$ enter equation (4.23) and thus any other eMZV of the same weight and length in the combination

$$
35 \gamma(8,6)+81 \gamma(10,4)
$$

exclusively. The above quantity is the first in a series of links to the derivation algebra $\mathfrak{u}$ introduced and discussed in the next subsection.

\subsection{A relation to the derivation algebra $\mathfrak{u}$}

The explanation of the deviating numbers for indecomposable eMZVs compared to shuffleindependent Eisenstein integrals in the last subsection can be provided starting from the following differential equation for the KZB associator $A(q)$ defined in equation (2.37) [1]: 
Table 4. Number of indecomposable eMZVs at length $\ell$ according to the counting of $\gamma\left(k_{1}, k_{2}, \ldots, k_{n}\right)$ suggested by the above observations (a), (b) and (c). The black numbers denote the number of shuffle-independent $\gamma$ 's with $k_{i}=0,4,6, \ldots$ and $k_{1} \neq 0$ while the red numbers indicate a deviating number of indecomposable eMZVs found from reflection-, shuffle-and Fay relations or the $q$-expansion.

న

\begin{tabular}{|c|c|c|c|c|c|c|c|c|c|c|c|c|c|c|c|c|c|c|c|}
\hline$w_{\omega}$ & 1 & 2 & 3 & 4 & 5 & 6 & 7 & 8 & 9 & 10 & 11 & 12 & 13 & 14 & 15 & 16 & 17 & 18 & 19 \\
\hline 2 & 1 & & 1 & & 1 & & 1 & & 1 & & 1 & & 1 & & 1 & & 1 & & 1 \\
\hline 3 & & 1 & & 1 & & 1 & & 2 & & 2 & & $3_{2}$ & & 3 & & $4_{3}$ & & $4_{3}$ & \\
\hline 4 & 1 & & 1 & & 2 & & 3 & & 4 & & $6_{5}$ & & $8_{7}$ & & $10_{8}$ & & $13_{10}$ & & $16_{12}$ \\
\hline 5 & & 1 & & 2 & & 4 & & 6 & & $10_{9}$ & & $14_{13}$ & & $21_{17}$ & & $28_{23}$ & & $39_{30}$ & \\
\hline
\end{tabular}




$$
\frac{\mathrm{d}}{\operatorname{dlog} q}\left(\mathrm{e}^{\mathrm{i} \pi[y, x]} A(q)\right)=\frac{1}{4 \pi^{2}}\left(\sum_{n=0}^{\infty}(2 n-1) G_{2 n}(q) \epsilon_{2 n}\right)\left(\mathrm{e}^{\mathrm{i} \pi[y, x]} A(q)\right) .
$$

The Eisenstein series $G_{2 n}$ in equation (4.25) are accompanied by derivations $\epsilon_{2 n}$ which act on the non-commutative variables $x$ and $y$ in the expansion of $A(q)$ via

$$
\begin{gathered}
\epsilon_{2 n}(x)=\left(a d_{x}\right)^{2 n}(y), \quad n \geqslant 0 \\
\epsilon_{2 n}(y)=\left[y,\left(a d_{x}\right)^{2 n-1}(y)\right]+\sum_{1 \leqslant j<n}(-1)^{j}\left[\left(a d_{x}\right)^{j}(y),\left(a d_{x}\right)^{2 n-1-j}(y)\right], \quad n>0 \\
\epsilon_{0}(y)=0 .
\end{gathered}
$$

They generate a Lie subalgebra $\mathfrak{u}$ of the algebra of all derivations on the free Lie algebra generated by $x, y[16,22,23]$. The relations originating from equation $(4.26 a)$ have been studied extensively in [24]. Beyond

$$
\left[\epsilon_{2 n}, \epsilon_{2}\right]=0, \quad n \geqslant 0,
$$

there are several non-obvious relations such as

$$
\begin{gathered}
0=\left[\epsilon_{10}, \epsilon_{4}\right]-3\left[\epsilon_{8}, \epsilon_{6}\right], \\
0=2\left[\epsilon_{14}, \epsilon_{4}\right]-7\left[\epsilon_{12}, \epsilon_{6}\right]+11\left[\epsilon_{10}, \epsilon_{8}\right], \\
0=80\left[\epsilon_{12},\left[\epsilon_{4}, \epsilon_{0}\right]\right]+16\left[\epsilon_{4},\left[\epsilon_{12}, \epsilon_{0}\right]\right]-250\left[\epsilon_{10},\left[\epsilon_{6}, \epsilon_{0}\right]\right]-125\left[\epsilon_{6},\left[\epsilon_{10}, \epsilon_{0}\right]\right] \\
+280\left[\epsilon_{8},\left[\epsilon_{8}, \epsilon_{0}\right]\right]-462\left[\epsilon_{4},\left[\epsilon_{4}, \epsilon_{8}\right]\right]-1725\left[\epsilon_{6},\left[\epsilon_{6}, \epsilon_{4}\right]\right] .
\end{gathered}
$$

The rôle of $\epsilon_{2}$ as a central element in equation (4.27) is reminiscent of the above observation (b): any appearance of the non-modular $G_{2}$ can be captured by powers of $\gamma(2)$. Moreover, a peculiar linear combination of $\gamma(8,6)$ and $\gamma(10,4)$ has been observed in equation (4.24) to appear in all eMZVs at $\ell_{\omega}=3$ and $w_{\omega}=12$. Upon identifying labels in $\gamma$ with those of derivations $\epsilon_{2 n}$ as suggested by equation (4.25), one could attribute the selection rule on $\gamma(8,6)$ and $\gamma(10,4)$ to equation $(4.28 a)$.

This connection will be made more precise in the subsequent. For this purpose, iterated Eisenstein integrals will be rewritten in terms of non-commutative letters similar to the ones discussed for usual MZVs in section 3. In particular we are led to an structure reminiscent of the $\phi$-map, which provided the key to a convenient representation of MZVs in which all known relations over $\mathbb{Q}$ are automatically built in.

The rewriting of the $\omega$-representation of eMZVs in terms of non-commutative letters turns out to mimick the procedure used in order to define the map $\phi$ in equation (3.6). Despite the resemblance, however, the definition of the map $\phi$ depends on the choice of an algebra basis for motivic MZVs, while the rewriting of eMZVs in terms of non-commutative letters to be described below is completely canonical.

4.3.1. Eisenstein integrals as non-commutative words. As a first step to make the connection between eMZVs and the algebra of derivations manifest, let us translate iterated Eisenstein integrals into words composed from non-commutative generators $g_{0}, g_{2}, g_{4}, \ldots$,

9 We are grateful to Francis Brown who helped us to understand the language and scope of non-commutative words in the context of multiple modular values, in particular for pointing us to section 12 of [18]. 


$$
\psi\left[\gamma\left(k_{1}, k_{2}, \ldots, k_{n}\right)\right] \equiv \frac{g_{k_{n}} g_{k_{n-1}} \ldots g_{k_{2}} g_{k_{1}}}{\prod_{j=1}^{n}\left(k_{j}-1\right)} .
$$

Here, we need to assume that the iterated Eisenstein integrals are linearly independent, and the normalization $g_{k} /(k-1)$ of the non-commutative alphabet is suggested by the combinations $(k-1) G_{k}$ in equation (4.25) and the factors of $n_{i} G_{n_{i}+1}$ in equation $(4.10 a)$.

The non-commutative letters $g_{k}$ are naturally endowed with a shuffle product. The $\psi$-map defined by equation (4.29) then satisfies

$\psi\left[\gamma\left(n_{1}, n_{2}, \ldots, n_{r}\right) \gamma\left(k_{1}, k_{2}, \ldots, k_{s}\right)\right]=\psi\left[\gamma\left(n_{1}, n_{2}, \ldots, n_{r}\right)\right] \omega \psi\left[\gamma\left(k_{1}, k_{2}, \ldots, k_{s}\right)\right]$.

The linear combination of $\gamma(8,6)$ and $\gamma(10,4)$ appearing in the eMZVs with $w_{\omega}=12$ and $\ell_{\omega}=3$ are mapped to

$$
\psi[35 \gamma(8,6)+81 \gamma(10,4)]=g_{6} g_{8}+3 g_{4} g_{10} .
$$

Hence, the image of any $w_{\omega}=12, \ell_{\omega}=3 \mathrm{eMZV}$ under equation (4.29) is annihilated by the differential operator

$$
\left[\partial_{10}, \partial_{4}\right]-3\left[\partial_{8}, \partial_{6}\right]
$$

once differentiation of a non-commutative word in $g_{i}$ is defined to act on the leftmost letter

$$
\partial_{j} g_{k_{1}} \ldots g_{k_{n}}=\delta_{j, k_{1}} g_{k_{2}} \ldots g_{k_{n}} .
$$

This differentiation rule satisfies a Leibniz property w.r.t. the shuffle product equation (4.30) and appeared already in the context of the representation of motivic MZVs in terms of noncommutative letters $f_{i}$ [11], see the discussion in section 3. Note furthermore that the recursive construction of the eMZVs' $\psi$-image via equation (4.9) with coefficients $\xi_{2 k}\left(n_{1}, \ldots, n_{r}\right)$ determined by the differential equation (2.47) is similar to the recursive evaluation of the $\phi$ map [11]: the coefficients $\xi_{2 k+1}$ of $\phi\left(\zeta^{\mathfrak{m}}\right)=\sum_{3 \leqslant 2 k+1 \leqslant w} f_{2 k+1} \xi_{2 k+1}$ for some motivic MZV of weight $w$ are determined by the component of weight $(2 k+1) \otimes(w-2 k-1)$ in the coaction. Hence, the $\tau$-derivative in the form equation (4.9) exhibits a formal similarity to the coaction of motivic MZVs.

However, there is an important difference between the $\phi$-map and the rewriting of eMZVs in its $\psi$-image: while the $\phi$-map depends on a choice of algebra generators (for example the adaptation of equation (3.10) to the basis in table 3), the $\psi$-map for eMZVs is completely canonical.

In summary, the $\psi$-image of an eMZV $\omega\left(n_{1}, \ldots, n_{r}\right)$ is computed in two steps:

- use the differential equation to write $\omega\left(n_{1}, \ldots, n_{r}\right)$ as a linear combination of iterated Eisenstein integrals $\gamma\left(k_{1}, \ldots, k_{s}\right)$. Relying on our working hypothesis that iterated Eisenstein integrals are linearly independent, this decomposition is unique.

- apply the map in equation (4.29) to each of the $\gamma$ 's.

4.3.2. Non-commutative differentiation and the derivation algebra $\mathfrak{u}$. The similarity between equations (4.28a) and (4.32) suggests to identify derivations $\epsilon_{2 m}$ with derivatives with respect to the non-commutative letters $\partial_{2 m}$. Indeed, we will verify in three steps that the derivations $\epsilon_{2 m}$ encode the action of $\partial_{2 m}$ on the $\psi$-image of the KZB associator equation (2.37) and therefore on the $\psi$-image of any eMZVs: 
(i) integrate the differential equation (4.25) of the KZB associator

$\mathrm{e}^{\mathrm{i} \pi[y, x]}(A(q)-A(0))=\mathrm{e}^{\mathrm{i} \pi[y, x]} \frac{1}{4 \pi^{2}} \sum_{n=0}^{\infty}(2 n-1) \int_{0}^{q} \operatorname{dlog} q^{\prime} G_{2 n}\left(q^{\prime}\right) \epsilon_{2 n} A\left(q^{\prime}\right)$,

using the corollary $\epsilon_{2 n}([y, x])=0$ of equation (4.26a) to commute $\epsilon_{2 n} \mathrm{e}^{\mathrm{i} \pi[y, x]}=\mathrm{e}^{\mathrm{i} \pi[y, x]} \epsilon_{2 n}$ (ii) apply the $\psi$-map defined in equation (4.29):

$$
\psi[A(q)-A(0)]=\sum_{n=0}^{\infty} \epsilon_{2 n} g_{2 n} \psi[A(q)],
$$

using the fact that integration against $\frac{(2 n-1)}{4 \pi^{2}} G_{2 n}$ amounts to left-concatenation with $g_{2 n}$ (iii) act with $\partial_{2 m}$ such that the sum over $n$ collapses by equation (4.33),

$$
\partial_{2 m} \psi[A(q)-A(0)]=\partial_{2 m} \psi[A(q)]=\partial_{2 m} \sum_{n=0}^{\infty} \epsilon_{2 n} g_{2 n} \psi[A(q)]=\epsilon_{2 m} \psi[A(q)],
$$

where we used that the derivative $\partial_{2 m}$ annihilates the boundary term $A(0)$, which translates into an empty word in the letters $g$.This is the reason, why any relation among the derivations $\epsilon_{i}$ defines a differential operator via $\epsilon_{i} \rightarrow \partial_{i}$ which annihilates the $\psi$-image of any eMZV. Explicitly:

$\forall E \in \mathfrak{u}$ such that $E(x)=E(y)=\left.0 \quad \Rightarrow \quad E\right|_{\epsilon_{2 m} \rightarrow \partial_{2 m}} \psi\left[\omega\left(n_{1}, \ldots, n_{r}\right)\right]=0$.

Thus, any relation in $\mathfrak{u}$ obstructs the appearance of some single linear combination of iterated Eisenstein integrals equation (4.2) among eMZVs and reduces the counting of indecomposable representatives at lengths and weights governed by the conversion rules equation $(4.17 a)$.

\subsection{Systematics of relations in the derivation algebra}

Naturally, we have been checking the implications of counting shuffle-independent $\gamma\left(k_{1}, k_{2}, \ldots, k_{n}\right)$ subject to $k_{1} \neq 0$ and $k_{i} \neq 2$ (see the three observations around equation (4.20)) and the connection with the derivation algebra $\mathfrak{u}$ established in the previous subsection by comparing $q$-expansions: up to weights $w_{\omega}=30,18,8,5$ for $\ell_{\omega}=3,4,5,6$ we find complete agreement. There are, however, no obstructions for repeating the analysis for eMZVs of higher length, as tested for several low weights at length 7 and 8.

Counting relations from the algebra of derivations $\mathfrak{u}$ for a given weight and depth works as follows: we start with an ansatz for a relation $E$ of the form

$$
0 \stackrel{!}{=} \sum_{\left\{n_{1}, n_{2}, \ldots, n_{r}\right\}} \alpha_{n_{1}, n_{2}, \ldots, n_{r}}\left[\left[\ldots\left[\left[\partial_{n_{1}}, \partial_{n_{2}}\right], \partial_{n_{3}}\right], \ldots\right], \partial_{n_{r}}\right]
$$

with rational fudge coefficients $\alpha_{n_{1}, n_{2}, \ldots, n_{r}}$ and $\left\{n_{1}, n_{2}, \ldots, n_{r}\right\}$ composed of $n_{i}=0,4,6, \ldots$ of appropriate weight and length. The number $r$ of partial derivatives in the nested commutators of equation (4.38) (or the number of $\epsilon_{n}$ in the dual derivations, respectively) is referred to as depth. Of course, the summation in equation (4.38) is restricted to nested commutators which are independent under Jacobi identities.

Considering equation (4.37), the above ansatz for $E$ should annihilate all $\psi$-images of eMZVs of the length and weight considered. Using a sufficiently large set of eMZVs, one can easily fix all fudge coefficients in the ansatz and thus extract relations.

Using this method, we find perfect agreement of equation (4.38) as an operator equation acting on eMZVs with the relations in the derivation algebra available in [24, 40, 41]. In the 
Table 5. Irreducible relations $r_{w}^{\ell}$. Up to weight 30 there are no more than two relations at a particular weight and length, which will, however, change proceeding to higher weight and length. An actual list of the first irreducible relations is available in appendix C.1.

\begin{tabular}{cccccccccc}
\hline \multicolumn{10}{c}{$w_{\gamma}$} \\
\hline$\ell_{\gamma}$ & 2 & 3 & 4 & 5 & 6 & 7 & 8 & 9 & 10 \\
\hline 12 & 0 & 0 & 0 & 0 & 0 & 0 & 0 & 0 & 0 \\
14 & $r_{14}^{2}$ & 0 & 0 & 0 & 0 & 0 & 0 & 0 & 0 \\
16 & 0 & $r_{16}^{3}$ & 0 & 0 & 0 & 0 & 0 & 0 & 0 \\
18 & $r_{18}^{2}$ & 0 & $r_{18}^{4}$ & 0 & 0 & 0 & 0 & 0 & 0 \\
20 & $r_{20}^{2}$ & $r_{20}^{3}$ & 0 & $r_{20}^{5}$ & 0 & 0 & 0 & 0 & 0 \\
22 & $r_{22}^{2}$ & $r_{22}^{3}$ & $r_{22}^{4}$ & 0 & $r_{22}^{6}$ & 0 & 0 & 0 & 0 \\
24 & $r_{24}^{2}$ & $r_{24}^{3}$ & $r_{24}^{4}$ & $r_{24}^{5}$ & 0 & $r_{24}^{7}$ & 0 & 0 & 0 \\
26 & $2 \times r_{26}^{2}$ & $r_{26}^{3}$ & $r_{26}^{4}$ & $r_{26}^{5}$ & $r_{26}^{6}$ & 0 & $r_{26}^{8}$ & 0 & 0 \\
28 & $r_{28}^{2}$ & $2 \times r_{28}^{3}$ & $r_{28}^{4}$ & $r_{28}^{5}$ & $r_{28}^{6}$ & $r_{28}^{7}$ & 0 & $r_{28}^{9}$ & 0 \\
30 & $2 \times r_{30}^{2}$ & $r_{30}^{3}$ & $2 \times r_{30}^{4}$ & $r_{30}^{5}$ & $r_{30}^{6}$ & $r_{30}^{7}$ & $r_{30}^{8}$ & 0 & $r_{30}^{10}$ \\
\hline
\end{tabular}

following paragraphs we will review their classification and extend the explicit results to higher commutator-depths.

4.4.1. Special rôle of $\epsilon_{2}$. As already observed above, none of the indecomposable eMZVs besides $\omega(0,1)$ does contain an Eisenstein integral involving $G_{2}$. This reflects the rôle of $\epsilon_{2}$ as a central element, as noted in equation (4.27). Hence, it is sufficient to study commutator relations without $\epsilon_{2}$.

4.4.2. Irreducible versus reducible relations. Any relation in the derivation algebra $\mathfrak{u} \ni E=0$ of the form equation (4.38) yields an infinity of higher-depth corollaries by repeated adjoint action of $\epsilon_{n}$ :

$$
E=0 \Rightarrow a d_{n_{1}, n_{2}, \ldots, n_{k}}(E) \equiv\left[\epsilon_{n_{1}},\left[\epsilon_{n_{2}},\left[\ldots,\left[\epsilon_{n_{k}}, E\right] \ldots\right]\right]\right]=0 .
$$

Any instance of equation (4.39) with $k>0$ and $E$ denoting a vanishing combination of $\epsilon_{n}$-commutators is called a reducible relation, whereas relations that cannot be cast into the form $a d_{n_{1}, n_{2}, \ldots, n_{k}}(E)=0$ are referred to as irreducible. For instance, the simplest non-obvious relation equation $(4.28 a)$ is irreducible and gives rise to reducible relations such as

$$
\left[\epsilon_{n},\left[\epsilon_{10}, \epsilon_{4}\right]-3\left[\epsilon_{8}, \epsilon_{6}\right]\right]=0
$$

and generalizations to higher depth. They affect the bookkeeping of irreducible eMZVs starting from $w_{\gamma}=14$ and $\ell_{\gamma}=3$, which corresponds to $w_{\omega}=11$ and $\ell_{\omega}=4$.

A correspondence between cusp forms of weight $w$ and irreducible relations at depth $d$ and weight $w+2(d-1)$ has been discussed in [24]. In the same way as the number of cusp forms at modular weight $w$ is given by 


$$
\chi_{w} \equiv\left\{\begin{array}{ll}
\left\lfloor\frac{w}{12}\right\rfloor-1 & : w=2 \bmod 12 \\
\left\lfloor\frac{w}{12}\right\rfloor & : \text { other even values of } w
\end{array},\right.
$$

we expect $\chi_{w-2(d-1)}$ irreducible relations at weight $w$ and depth $d$ relevant to eMZVs of nonnegative weight $w_{\omega}$ (see equation (4.16) for its relation to the weight of the iterated Eisenstein integral). In table 5, this conjectural counting is exemplified up to $w_{\gamma}=30$ with a notation $r_{w_{\gamma}}^{d}$ for such irreducible relations. Relations of depth two can be cast into a closed formula [26]

$0=\sum_{i=1}^{2 n+2 p-1} \frac{\left[\epsilon_{2 p+2 n-i+1}, \epsilon_{i+1}\right]}{(2 p+2 n-i-1) !}\left\{\frac{(2 n-1) ! B_{i-2 p+1}}{(i-2 p+1) !}+\frac{(2 p-1) ! B_{i-2 n+1}}{(i-2 n+1) !}\right\}$

where $p, n \geqslant 1$ denote arbitrary integers and $B_{n}$ are Bernoulli numbers. Each term of equation (4.42) carries weight $2(p+n+1)$, e.g. the weight-14 relation equation (4.28a) follows from any partition of $p+n=6$, and the weight-18 relation equation $(4.28 b)$ from any partition of $p+n=8$.

Irreducible relations at higher depth can be obtained in electronic form from the website https://tools.aei.mpg.de/emzv, whereas relations of depth three at $w=16,20$ and depth four at $w=18,22$ are provided in [24]. New relations beyond those in said reference are obtained from the differential operators equation (4.38) annihilating all eMZVs of corresponding weight and length. This approach to finding relations in the derivation algebra appears computationally more efficient to us than evaluating the action of elements of the derivation algebra on generators $x$ and $y$ of the free Lie algebra. However, once a candidate relation has been identified, it is straightforward to check its validity using its action on the letters $x$ and $y$ via equation (4.26a).

4.4.3. Vanishing nested commutators. Starting from $w_{\gamma}=8$ and $\ell_{\gamma}=5$, we find that the $\psi$ image of any eMZV with appropriate weight and length is annihilated by operators of the form

$$
\left[\left[\left[\left[\partial_{4}, \partial_{0}\right], \partial_{0}\right], \partial_{0}\right], \partial_{2 m}\right] \text {. }
$$

The reason becomes clear by considering $\gamma(4,0,0,0)$, one of the corresponding Eisenstein integrals. By equation (4.16), related eMZVs are bound to have $\ell_{\omega}=5$ and $w_{\omega}=0$, but the only eMZV with these properties is $\omega(0,0,0,0,0)=1 / 120$ which cannot equal the nonconstant $\gamma(4,0,0,0)$. Hence, the latter does not occur among eMZVs and signals the irreducible relation

$$
\left[\left[\left[\epsilon_{4}, \epsilon_{0}\right], \epsilon_{0}\right], \epsilon_{0}\right]=0,
$$

which in turn implies that $\left[\left[\left[\partial_{4}, \partial_{0}\right], \partial_{0}\right], \partial_{0}\right]$ annihilates the KZB associator by equation (4.36). The relation equation (4.44) can be understood from the organization of $\mathfrak{u}$ in terms of representations of the Lie algebra $\mathfrak{s l}_{2}$ : considering $\epsilon_{2 m}$ as the lowest-weight state in a $(2 m-1)$-dimensional module, the highest-weight vector $a d_{0}^{2 m-2} \epsilon_{2 m}$ is annihilated by further adjoint action of $\epsilon_{0}$.

Further irreducible relations of this type include

$$
a d_{0}^{p-1} \epsilon_{p}=\underbrace{\left[\epsilon_{0}, \ldots\left[\epsilon_{0},\left[\epsilon_{0}, \epsilon_{p}\right]\right] \ldots\right]}_{p-1 \text { times }}=0, \quad p=4,6,8, \ldots,
$$

corresponding to the Eisenstein integral $\gamma\left(p, 0^{p-1}\right)$ with would-be eMZV partners of vanishing $w_{\omega}$. Different partitions of the weight in equation (4.45) lead to further relations 
such as

$$
\begin{aligned}
& {\left[\left[\left[\left[\left[\left[\left[\epsilon_{4}, \epsilon_{0}\right], \epsilon_{4}\right], \epsilon_{0}\right], \epsilon_{0}\right], \epsilon_{0}\right], \epsilon_{0}\right], \epsilon_{0}\right]=0,} \\
& \text { [[[[[[[[[ } \left.\left.\left.\left.\left.\left.\left.\left.\left.\epsilon_{4}, \epsilon_{0}\right], \epsilon_{6}\right], \epsilon_{0}\right], \epsilon_{0}\right], \epsilon_{0}\right], \epsilon_{0}\right], \epsilon_{0}\right], \epsilon_{0}\right], \epsilon_{0}\right]=0 \text {. }
\end{aligned}
$$

Since all their permutations via $\epsilon_{4} \leftrightarrow \epsilon_{0}$ or $\epsilon_{6} \leftrightarrow \epsilon_{0}$ can be identified as a reducible relation descending from equation (4.45), we expect no further irreducible relations at $d=w_{\gamma}=8$ or 10 besides equation (4.46).

4.4.4. Additional generators of the Lie algebra. Consider the free Lie algebra $\mathfrak{k}=\mathbb{L}\left(z_{3}, z_{5}, z_{7}, \ldots\right)$ generated by one element in every odd degree strictly greater than one. As mentioned on page 6 of [24], every generator $z_{2 k+1}$ of $\mathfrak{k}$ defines a derivation $\widetilde{z}_{2 k+1}$ of depth $2 k+1$ and weight $4 k+2$ of the free Lie algebra on two generators $x, y$, and satisfies $\left[\widetilde{z}_{2 k+1}, \mathfrak{u}\right] \subset \mathfrak{u}$. More precisely, the elements $\epsilon_{0}, \epsilon_{2}$ are annihilated by the elements $\widetilde{z}_{2 k+1}$

$$
0=\left[\widetilde{z}_{2 k+1}, \epsilon_{0}\right]=\left[\widetilde{z}_{2 k+1}, \epsilon_{2}\right], \quad k=1,2,3, \ldots,
$$

and their commutators with $\epsilon_{4}, \epsilon_{6}, \ldots$ can be constructed using the techniques of [24], e.g. $\left[\widetilde{z}_{3}, \epsilon_{4}\right]=-\frac{1}{14}\left[\left[\epsilon_{4}, \epsilon_{0}\right],\left[\epsilon_{6}, \epsilon_{0}\right]\right]+\frac{1}{42}\left[\epsilon_{4},\left[\epsilon_{0},\left[\epsilon_{0}, \epsilon_{6}\right]\right]\right]-\frac{1}{7}\left[\epsilon_{6},\left[\epsilon_{0},\left[\epsilon_{0}, \epsilon_{4}\right]\right]\right]$.

They give rise to further reducible relations, starting from length five at weights $20,24,26, \ldots$ by the commutator of $\widetilde{z_{3}}$ with the depth-two relations in (4.28) or equation (4.42).

\subsection{Counting relations between nested commutators}

4.5.1. Example. In order to demonstrate the virtue of the derivation algebra as a counting formalism for indecomposable eMZVs, let us consider $w_{\gamma}=20, \ell_{\gamma}=5$ as a specific example, which corresponds to $w_{\omega}=15, \ell_{\omega}=6$. This is the first situation, where all four types of relations described in the previous section have to be taken into account in order to arrive at what we believe is the correct counting of eMZVs.

The naïve enumeration of shuffle-independent $\gamma$ 's with $k_{1} \neq 0$ and $k_{i} \neq 2$ leads to 55 distinct elements. Each relation of depth 5 and weight 20 in the derivation algebra will lower this number according to equation (4.37).

Let us first consider reducible relations. Starting from table 5, one can construct the following reducible relations by adjoint action of $\epsilon_{n}$ (recalling the notation $r_{w_{\gamma}}^{d}$ for irreducible relations of depth $d$ and weight $w_{\gamma}$ as well as $\left.a d_{n_{1}, n_{2}, \ldots, n_{k}} r_{i}^{j} \equiv\left[\epsilon_{n_{1}},\left[\epsilon_{n_{2}},\left[\ldots,\left[\epsilon_{n_{k}}, r_{w_{\gamma}}^{d}\right] \ldots\right]\right]\right]\right)$ :

$$
\begin{aligned}
& a d_{6,0,0} r_{14}^{2} \leftrightarrow 3 \text { permutations, } \quad a d_{0,0,0} r_{20}^{2} \leftrightarrow 1 \text { permutation } \\
& a d_{4,0} r_{16}^{3} \leftrightarrow 2 \text { permutations, } \quad a d_{0,0} r_{20}^{3} \leftrightarrow 1 \text { permutation. }
\end{aligned}
$$

In addition, there is one relation each descending from the vanishing nested commutator equation (4.44) and the additional Lie algebra generator $\widetilde{z_{3}}$,

$$
\left[\left[\left[\left[\epsilon_{4}, \epsilon_{0}\right], \epsilon_{0}\right], \epsilon_{0}\right], \epsilon_{16}\right]=0 \text { and }\left[\widetilde{z}_{3}, r_{14}^{2}\right]=0 \text {, }
$$

which makes a total of 9 reducible relations.

Indeed, starting with an ansatz of the form equation (4.38), we find ten distinct relations: while equations (4.49) and (4.50) are confirmed, our method explicitly delivers the new irreducible relation $r_{20}^{5}$ expected from table 5. To our knowledge this is the first appearance of an explicit relation at depth 5 in $\mathfrak{u}$, which is written out in appendix C.5. Correspondingly, 
Table 6. Shuffle-independent $\gamma\left(k_{1}, \ldots, k_{n}\right)$ subject to $k_{1} \neq 0$ and $k_{i} \neq 2$ at various weights $w_{\gamma}$ and lengths $\ell_{\gamma}$.

\begin{tabular}{ccccccccccccccccc}
\hline \multicolumn{11}{c}{$\ell_{\gamma}$} \\
\hline$w_{\gamma}$ & 0 & 2 & 4 & 6 & 8 & 10 & 12 & 14 & 16 & 18 & 20 & 22 & 24 & 26 & 28 & 30 \\
\hline 1 & 1 & 1 & 1 & 1 & 1 & 1 & 1 & 1 & 1 & 1 & 1 & 1 & 1 & 1 & 1 & 1 \\
2 & 0 & 0 & 1 & 1 & 1 & 2 & 2 & 3 & 3 & 4 & 4 & 5 & 5 & 6 & 6 & 7 \\
3 & 0 & 0 & 1 & 1 & 2 & 3 & 4 & 6 & 8 & 10 & 13 & 16 & 19 & 23 & 27 & 31 \\
4 & 0 & 0 & 1 & 1 & 2 & 4 & 6 & 10 & 14 & 21 & 28 & 39 & 50 & 66 & 82 & 104 \\
5 & 0 & 0 & 1 & 1 & 3 & 5 & 9 & 15 & 24 & 37 & 55 & 80 & 113 & 156 & 211 & 280 \\
6 & 0 & 0 & 1 & 1 & 3 & 6 & 11 & 21 & 35 & 59 & 93 & 146 & 217 & 322 & 459 & 649 \\
7 & 0 & 0 & 1 & 1 & 4 & 7 & 15 & 28 & 51 & 89 & 150 & 245 & 389 & 602 & 910 & 1347 \\
\hline
\end{tabular}

we find the number of indecomposable eMZVs at $\left(\ell_{\gamma}, w_{\gamma}\right)=(5,20)\left(\right.$ or $\left.\left(\ell_{\omega}, w_{\omega}\right)=(6,15)\right)$ to be 45 .

4.5.2. General. In order to repeat the counting procedure from the above example for a variety of weights and lengths, the following tables give an overview of the required ingredients: The numbers of shuffle-independent iterated Eisenstein integrals compatible with observations (a) and (b) in section 4.2 are gathered in table 6 and have to be compared with the counting of relations in $\mathfrak{u}$ seen in table 7 . Once the offset between $\left(w_{\gamma}, \ell_{\gamma}\right)$ and $\left(w_{\omega}, \ell_{\omega}\right)$ in equation $(4.17 a)$ is taken into account, one arrives at the numbers of indecomposable eMZVs in the $\omega$-representation noted in table 8 .

From the above data, one readily arrives at all-weight statements on the number of indecomposable eMZVs of length $\ell_{\omega} \leqslant 4$ :

- At length $\ell_{\omega}=2$, there is obviously one indecomposable eMZV at each odd weight $w_{\omega}$.

- At length $\ell_{\omega}=3$, the number of indecomposable eMZVs at even weight $w_{\omega}$ is $\left\lceil\frac{1}{6} w_{\omega}\right\rceil$. This follows from comparing the number $\left\lceil\frac{w_{\omega}}{4}\right\rceil-1$ of admissible $\gamma\left(k_{1}, k_{2}\right)$ $\left(k_{1}>k_{2}, k_{i} \neq 2\right.$ ) at weight $w_{\omega}>4$ with the counting of depth-two relations in $\mathfrak{u}$ governed by equation (4.41).

- At length $\ell_{\omega}=4$, the number of indecomposable eMZVs at odd weight $w_{\omega}$ is conjectured to be $\left[\frac{1}{2}+\frac{1}{48}\left(w_{\omega}+5\right)^{2}\right\rfloor$. This conjecture stems from extrapolating [42] the data available at $w_{\omega} \leqslant 37$. The extrapolation will remain valid, if the counting of irreducible $r_{w}^{3}$ keeps on following the cusp forms.

Starting from the next length, $\ell_{\omega}=5$ or $\ell_{\gamma}=4$, an effect well-known from the algebra of MZVs kicks in: because the lowest non-trivial relation from the derivation algebra $\mathfrak{u}$ exists at weight 14 depth 2 , there is the possibility to obtain the 'relation of a relation' $\operatorname{ad}_{r_{14}^{2}}\left(r_{14}^{2}\right)=0$ at weight 28, depth 4 . This effect, which appears in iterated form for higher depth, as well as the action of the generators of the free Lie algebra $\mathfrak{k}$ described in section 4.4 render the counting at higher depth difficult. Correspondingly, a closed formula, e.g. a generating series for the number of indecomposable eMZVs at given length and weight is still lacking and some of the entries in table 7 are left undetermined. 
Table 7. Relations in the derivation algebra at various weights $w_{\gamma}$ and depths $d$, excluding the central element $\epsilon_{2}$. 
Table 8. Numbers $N\left(\ell_{\omega}, w_{\omega}\right)$ of indecomposable eMZVs in their $\omega$-representation. This is an extended version of table 2 , where the black results are obtained by explicitly determining $q$-expansions while results printed in blue originate from testing relations between nested commutators as described around equation (4.38).

$\stackrel{\omega}{\circlearrowleft}$

\begin{tabular}{|c|c|c|c|c|c|c|c|c|c|c|c|c|c|c|c|c|c|c|c|c|c|c|c|}
\hline$w_{\omega}$ & 1 & 2 & 3 & 4 & 5 & 6 & 7 & 8 & 9 & 10 & 11 & 12 & 13 & 14 & 15 & 16 & 17 & 18 & 19 & 20 & 21 & 22 & 23 \\
\hline 2 & 1 & & 1 & & 1 & & 1 & & 1 & & 1 & & 1 & & 1 & & 1 & & 1 & & 1 & & 1 \\
\hline 3 & & 1 & & 1 & & 1 & & 2 & & 2 & & 2 & & 3 & & 3 & & 3 & & 4 & & 4 & \\
\hline 4 & 1 & & 1 & & 2 & & 3 & & 4 & & 5 & & 7 & & 8 & & 10 & & 12 & & 14 & & 16 \\
\hline 5 & & 1 & & 2 & & 4 & & 6 & & 9 & & 13 & & 17 & & 23 & & 30 & & 37 & & 47 & \\
\hline 6 & 1 & & 2 & & 4 & & 8 & & 13 & & 22 & & 31 & & 45 & & $?$ & & $?$ & & $?$ & & $?$ \\
\hline 7 & & 1 & & 4 & & 8 & & 16 & & 29 & & 48 & & ? & & ? & & ? & & $?$ & & $?$ & \\
\hline
\end{tabular}




\subsection{A simpler representation of the eMZV subspace}

From the discussion in the previous subsections it became clear that eMZVs can be nicely represented in terms of iterated Eisenstein integrals equation (4.2). While those integrals have to be regularized individually as pointed out in the context of equation (4.7), the representation of eMZVs cannot involve any divergences upon integrating their $\tau$-derivative equation (2.47). In this section we would like to manifest this property and define a modified version of iterated Eisenstein integrals $\gamma_{0}$, which are individually convergent by construction. By using the $\gamma_{0}$-language, one will trade some of the connections to periods and motives [18] inherent in the $\gamma$-language for compactness of representation. A further advantage of the $\gamma_{0}$-language to be introduced is a better accessibility of the $q$-expansions of eMZVs.

4.6.1. Modified iterated Eisenstein integrals. Already in section 4.2 it was remarked that the $\tau$-derivative of eMZVs determined by the differential equation (2.47) cannot contain any constant terms. Therefore, it is an obvious idea to subtract the constants from the non-trivial Eisenstein series before defining their iterated integrals:

$$
\begin{aligned}
G_{0}^{0} \equiv & -1 \\
G_{k}^{0} \equiv & G_{k}-2 \zeta_{k}=\frac{2(-1)^{k / 2}(2 \pi)^{k}}{(k-1) !} \\
& \times \sum_{m, n=1}^{\infty} m^{k-1} q^{m n}, \quad k \text { even, } k \neq 0 .
\end{aligned}
$$

Using this definition, one can rewrite equations (4.8a) and (4.8b) as

$$
\begin{aligned}
& \frac{\mathrm{d}}{\mathrm{d} \log q} \omega(0, n)=\frac{n}{4 \pi^{2}} G_{n+1}^{0}, \quad n \text { odd } \\
& \frac{\mathrm{d}}{\operatorname{dlog} q} \omega(0,0, n)=\frac{n}{4 \pi^{2}} \omega(0, n+1) G_{0}^{0}, \quad n \text { even, }
\end{aligned}
$$

and the differential equation (2.47) for generic eMZVs can be easily cast into the form

$$
\frac{\mathrm{d}}{\operatorname{dlog} q} \omega\left(n_{1}, n_{2}, \ldots, n_{r}\right)=\frac{1}{4 \pi^{2}} \sum_{k=0}^{\infty} \rho_{2 k}\left(n_{1}, n_{2}, \ldots, n_{r}\right) G_{2 k}^{0} .
$$

In complete analogy to equation (4.9), the coefficients $\rho_{2 k}\left(n_{1}, \ldots, n_{r}\right)$ are linear combinations of eMZVs with weight $n_{1}+\ldots+n_{r}+1-2 k$ and length $r-1$, the only difference being that Eisenstein series in equation (2.47) are now expanded via $G_{k}=G_{k}^{0}-2 \zeta_{k} G_{0}^{0}$ whenever $k \neq 0$.

From the form equation (4.53) of the differential equation (2.47), it is straightforward to introduce modified iterated Eisenstein integrals $\gamma_{0}$ via

$$
\begin{gathered}
\gamma_{0}\left(k_{1}, k_{2}, \ldots, k_{n} ; q\right) \equiv \frac{1}{4 \pi^{2}} \int_{0 \leqslant q^{\prime} \leqslant q} \operatorname{dlog} q^{\prime} \gamma_{0}\left(k_{1}, \ldots, k_{n-1} ; q^{\prime}\right) G_{k_{n}}^{0}\left(q^{\prime}\right), \quad k_{1} \neq 0 \\
=\frac{1}{\left(4 \pi^{2}\right)^{n}} \int_{0 \leqslant q_{i}<q_{i+1} \leqslant q} \operatorname{dlog} q_{1} G_{k_{1}}^{0}\left(q_{1}\right) \operatorname{dlog} q_{2} G_{k_{2}}^{0}\left(q_{2}\right) \ldots \operatorname{dlog} q_{n} G_{k_{n}}^{0}\left(q_{n}\right),
\end{gathered}
$$

whose definition as an iterated integral implies

$$
\frac{\mathrm{d}}{\operatorname{dlog} q} \gamma_{0}\left(k_{1}, k_{2}, \ldots, k_{n} ; q\right)=\frac{G_{k_{n}}^{0}(q)}{4 \pi^{2}} \gamma_{0}\left(k_{1}, k_{2}, \ldots, k_{n-1} ; q\right),
$$


$\gamma_{0}\left(n_{1}, n_{2}, \ldots, n_{r} ; q\right) \gamma_{0}\left(k_{1}, k_{2}, \ldots, k_{s} ; q\right)=\gamma_{0}\left(\left(n_{1}, n_{2}, \ldots, n_{r}\right) ш\left(k_{1}, k_{2}, \ldots, k_{s}\right) ; q\right)$.

The notion of weight and length are not altered w.r.t. the definition for $\gamma$. Naturally, $\gamma_{0}$ 's suffer from the same caveat with respect to linear independence as their cousins $\gamma$. There are several advantages of employing this modified class of iterated Eisenstein integrals $\gamma_{0}$ for the description of eMZVs:

- Logarithmic divergences for $q \rightarrow 0$ as present in equation (4.2) do not occur after setting $k_{1} \neq 0$. Modified iterated Eisenstein integrals $\gamma_{0}$ are perfectly well-defined objects which do not require regularization.

- The number of terms necessary to express eMZVs as combinations of iterated Eisenstein integrals $\gamma_{0}$ is significantly lower than for $\gamma$.

- The absence of constant terms in the expansion of $G_{k_{1}}^{0}$ propagates to any convergent iterated Eisenstein integral,

$$
\gamma_{0}\left(k_{1}, k_{2}, \ldots, k_{n} ; 0\right)=0 .
$$

Note that we will again suppress the dependence on $q$ in most cases: $\gamma_{0}(\ldots) \equiv \gamma_{0}(\ldots ; q)$.

4.6.2. Examples. Let us return to the examples equations $(4.52 a)$ and $(4.52 b)$. The differential equation (4.55) immediately implies

$$
\begin{aligned}
& \omega(0, n)=\delta_{1, n} \frac{\pi \mathrm{i}}{2}+n \gamma_{0}(n+1), \quad n \text { odd, } \\
& \omega(0,0, n)=-\frac{1}{3} \zeta_{n}-n(n+1) \gamma_{0}(n+2,0), \quad n \text { even, }
\end{aligned}
$$

where $\delta_{1, n} \frac{\pi \mathrm{i}}{2}$ and $-\frac{1}{3} \zeta_{n}$ arise as integration constants w.r.t. $\log q$. Indeed, these expressions are convergent by definition and shorter than their counterparts in equations (4.10a) and (4.10b).

For illustrational purposes let us also revisit the example $\omega(0,3,5)$. Its derivative

$$
4 \pi^{2} \frac{\mathrm{d}}{\operatorname{dlog} q} \omega(0,3,5)=30 \zeta_{6} \omega(0,3)-15\left(G_{4}^{0}-2 \zeta_{4} G_{0}^{0}\right) \omega(0,5)+45 \omega(0,9)
$$

amounts to $\rho_{4}(0,3,5)=-15 \omega(0,5) \quad$ and $\quad \rho_{0}(0,3,5)=30 \zeta_{4} \omega(0,5)-45 \omega(0,9)-$ $30 \zeta_{6} \omega(0,3)$ in the notation of equation (4.9) and can be translated to modified Eisenstein integrals via equation $(4.58 a)$ :

$4 \pi^{2} \frac{\mathrm{d}}{\mathrm{d} \log q} \omega(0,3,5)=90 \zeta_{6} \gamma_{0}(4)-75\left(G_{4}^{0}-2 \zeta_{4} G_{0}^{0}\right) \gamma_{0}(6)+405 \gamma_{0}(10)$.

Integration using equation (4.54) yields the following alternative representation to equation (4.15),

$\omega(0,3,5)=-90 \zeta_{6} \gamma_{0}(4,0)+150 \zeta_{4} \gamma_{0}(6,0)-75 \gamma_{0}(6,4)-405 \gamma_{0}(10,0)$.

Further examples of eMZVs expressed in the language of modified iterated Eisenstein integrals can be found in appendix B.

4.6.3. q-expansion. In contrast to the $\gamma$-language used in the last section, there is no caveat on regularization when performing the integrals over $q_{j}$ in the definition equation (4.54) of $\gamma_{0}$. The $q$-expansion stems from the expression for Eisenstein series in equation (4.51) and can be cast into a closed form (with $0^{n}$ denoting a sequence of $n$ entries $0,0, \ldots, 0$ ): 


$$
\begin{aligned}
\gamma_{0}\left(k_{1},\right. & \left.0^{p_{1}-1}, k_{2}, 0^{p_{2}-1}, \ldots, k_{r}, 0^{p_{r}-1}\right)=\prod_{j=1}^{r}\left(-\frac{2(2 \pi \mathrm{i})^{k_{j}-2 p_{j}}}{\left(k_{j}-1\right) !}\right) \\
& \times \sum_{m_{i}, n_{i}=1}^{\infty} \frac{m_{1}^{k_{1}-1} m_{2}^{k_{2}-1} \ldots m_{r}^{k_{r}-1} q^{m_{1} n_{1}+m_{2} n_{2}+\ldots+m_{r} n_{r}}}{\left(m_{1} n_{1}\right)^{p_{1}}\left(m_{1} n_{1}+m_{2} n_{2}\right)^{p_{2}} \ldots\left(m_{1} n_{1}+m_{2} n_{2}+\ldots+m_{r} n_{r}\right)^{p_{r}}} .
\end{aligned}
$$

An even more compact representation can be achieved using the divisor sum

$$
\sigma_{k}(n) \equiv \sum_{d \mid n} d^{k}
$$

which allows to rewrite equation (4.62) as

$$
\begin{aligned}
\gamma_{0}\left(k_{1}, 0^{p_{1}-1}, k_{2}, 0^{p_{2}-1}, \ldots, k_{r}, 0^{p_{r}-1}\right)=\prod_{j=1}^{r}\left(-\frac{2(2 \pi \mathrm{i})^{k_{j}-2 p_{j}}}{\left(k_{j}-1\right) !}\right) \\
\quad \times \sum_{0<n_{1}<n_{2}<\cdots<n_{r}} \frac{\sigma_{k_{1}-1}\left(n_{1}\right) \sigma_{k_{2}-1}\left(n_{2}-n_{1}\right) \ldots \sigma_{k_{r}-1}\left(n_{r}-n_{r-1}\right) q^{n_{r}}}{n_{1}^{p_{1}} n_{2}^{p_{2}} \ldots n_{r}^{p_{r}}} .
\end{aligned}
$$

The above expression bears some resemblance to the sum representation equation (2.1) of MZVs. One could wonder if rearrangements of the sums could yield a genus-one analogue of stuffle relations. However, both the appearance of the divisor sums and the $q$-dependence prevent such manipulations. In fact, we did not observe a single relation among iterated Eisenstein integrals $\gamma_{0}$ beyond the shuffle relations equation (4.4) up to weights $44,31,22,19$ for length $2,3,4,5$, respectively.

Given the above $\gamma_{0}$-representation of the simplest eMZVs, we arrive at two closed forms for $q$-expansions

$$
\begin{aligned}
& \omega(0, k)=\delta_{k, 1} \frac{\mathrm{i} \pi}{2}+\frac{2(-1)^{(k+1) / 2}(2 \pi)^{k-1}}{(k-1) !} \sum_{m, n=1}^{\infty} \frac{m^{k-1}}{n} q^{m n}, \quad k \text { odd } \\
& \omega(0,0, k)=-\frac{1}{3} \zeta_{k}+\frac{2(-1)^{(k+2) / 2}(2 \pi)^{k-2}}{(k-1) !} \sum_{m, n=1}^{\infty} \frac{m^{k-1}}{n^{2}} q^{m n}, \quad k \text { even, }
\end{aligned}
$$

while further expressions for interesting $\omega(0,0, \ldots, 0, k)$ at higher length are given in appendix B.1.

4.6.4. Connection with the derivation algebra. A manifestly convergent description of eMZVs in terms of modified iterated Eisenstein integrals $\gamma_{0}$ comes with a price at the end of the day: the constant terms which have been omitted in the definition (4.54) have to be restored in order to establish a connection with the derivation algebra. In particular, the translation of modified iterated Eisenstein integrals into the language of non-commutative words built from letters $g_{k}$ described in subsection 4.3 involves various shifts $\sim \zeta_{k} g_{0}$,

$$
\begin{aligned}
& \psi\left[\gamma_{0}\left(k_{1}, 0^{p_{1}}, k_{2}, 0^{p_{2}}, \ldots, k_{n}, 0^{p_{n}}\right)\right]=(-1)^{p_{1}+p_{2}+\ldots+p_{n}} \\
& \quad \times\left(g_{0}\right)^{p_{n}}\left(\frac{g_{k_{n}}}{k_{n}-1}-2 \zeta_{k_{n}} g_{0}\right) \cdots\left(g_{0}\right)^{p_{2}}\left(\frac{g_{k_{2}}}{k_{2}-1}-2 \zeta_{k_{2}} g_{0}\right)\left(g_{0}\right)^{p_{1}}\left(\frac{g_{k_{1}}}{k_{1}-1}-2 \zeta_{k_{1}} g_{0}\right),
\end{aligned}
$$

where $\left(g_{0}\right)^{n}$ refers to $n$ adjacent letters $g_{0}$. Furthermore, the concatenation of words is understood to act linearly, e.g. $g_{2}\left(\zeta_{4} g_{0}+g_{4}\right) g_{8}=\zeta_{4} g_{2} g_{0} g_{8}+g_{2} g_{4} g_{8}$. Nevertheless, the counting of indecomposable eMZVs remains unmodified when projecting to the maximal component of their $\gamma$-representation, see the discussion below equation $(4.17 a)$. 


\section{Conclusions}

In this work we have been studying the systematics of relations between eMZVs. Our results support the conjecture that the entirety of relations can be traced back to reflection, shuffle and Fay identities.

The numbers $N\left(\ell_{\omega}, w_{\omega}\right)$ of indecomposable eMZVs at any weight and length can be explained once their connection to a special derivation algebra is taken into account: Any eMZV can be expressed in terms of iterated integrals over Eisenstein series whose appearance in turn is governed by the derivation algebra.

Our results for the numbers $N\left(\ell_{\omega}, w_{\omega}\right)$ of indecomposable eMZVs for various weights $w_{\omega}$ and lengths $\ell_{\omega}$ are listed in table 8 . In addition, there are all-weight formulæ available for $\ell_{\omega} \leqslant 4$ and odd values of $w_{\omega}+\ell_{\omega}$,

$$
N\left(2, w_{\omega}\right)=1, \quad N\left(3, w_{\omega}\right)=\left\lceil\frac{1}{6} w_{\omega}\right\rceil, \quad N\left(4, w_{\omega}\right)=\left\lfloor\frac{1}{2}+\frac{1}{48}\left(w_{\omega}+5\right)^{2}\right\rfloor,
$$

where the expression for $N\left(4, w_{\omega}\right)$ is conjectural. Because of the diversity of constraints originating from the derivation algebra as described in section 4 , a closed formula for all weights and lengths is challenging to find and not yet available. A closely related issue is the computation of the dimensions of the Lie algebra $\mathfrak{u}$, which has been carried out by Brown for depths 1,2 and 3 [43].

Explicit $q$-expansions for eMZVs are accessible using a slightly modified version of iterated Eisenstein integrals described in section 4.6. The resulting closed expression can be found in equation (4.64).

The improved understanding of eMZVs raises a variety of follow-up questions, starting with a connection of the underlying elliptic iterated integrals with recent results on Feynman integrals [6-9]. In particular, the techniques which led to the $q$-expansions of eMZVs furnish a convenient starting point to connect with the functions ELi introduced in [7] and generalized in [9].

The appearance of eMZVs in one-loop scattering amplitudes of the open superstring [5] suggested a systematic study of indecomposable eMZVs. The results of the current article should pave the way towards a compact form of string corrections at higher orders in $\alpha^{\prime}$ and might even lead to a glimpse of an all-order pattern. The existence of such a description is not unlikely: for open-string tree-level amplitudes a recursive formula based on the Drinfeld associator is known. It was found by extending an initial observation in [44] into a recursive computation of the complete $\alpha^{\prime}$-expansions in [45]. Similarly, the $\alpha^{\prime}$-expansion at one-loop might be accessible by using the elliptic associators discussed in [16].

The $\alpha^{\prime}$-expansion of the closed-string four-point amplitude at genus one has been investigated in [46-48], see $[49,50]$ for generalizations to five external states. The functions appearing in those amplitudes include non-holomorphic Eisenstein series and a variety of their generalizations which have been analyzed in [48]. It would be interesting to establish a connection between these non-holomorphic functions and modular-invariant combinations of eMZVs and their counterpart originating from the other homology cycle.

\section{Acknowledgments}

We are grateful to Carlos Mafra for collaboration in early stages of the project and on related topics. We would like to thank Aaron Pollack for helpful email correspondence regarding derivation algebras. Furthermore we are grateful to Axel Kleinschmidt, Michael Green, Eric 
D'Hoker for helpful discussions. In particular, we would like to acknowledge two elaborate discussions with Francis Brown in which he kindly explained to us-among other thingsthe connection to [18]. Moreover, we are indebted to an anonymous referee for a variety of very valuable and profound suggestions on an earlier version of this paper. Furthermore, we would like to thank Michael Green for an invitation to Cambridge and the Department of Applied Mathematics and Theoretical Physics of the University of Cambridge for hospitality. We acknowledge financial support by the European Research Council Advanced Grant No. 247252 of Michael Green. JB and NM would like to thank the Albert-Einstein-Institute for hospitality. Finally, we would like to acknowledge the Mainz Institute for Theoretical Physics (MITP) for its hospitality and its partial support during completion of this work. Moreover, we are grateful to the participants of the MITP workshop 'Amplitudes, Motives and Beyond', in particular Herbert Gangl and Anton Mellit, for numerous discussions about elliptic multiple zeta values and related topics.

\section{Appendix A. eMZV relations}

\section{A.1. Decomposition of boring eMZVs}

By equation (2.17), all the above examples of shuffle-reductions of boring eMZVs can be identified as special cases of the following general identity

$$
\left.\omega(B)\right|_{\text {boring }}=\sum_{k=1}^{\infty} D_{2 k} \sum_{\substack{B=A_{1} A_{2} \ldots A_{2 k} \\ \omega\left(A_{i}\right) \text { interesting }}} \omega\left(A_{1}\right) \omega\left(A_{2}\right) \ldots \omega\left(A_{2 k}\right),
$$

whose rational coefficients $D_{2}=\frac{1}{2}, D_{4}=-\frac{1}{8}, \ldots$ are given by

$$
D_{2 k}=(-1)^{k-1} \frac{(2 k-3) ! !}{k ! 2^{k}} .
$$

The arguments $B \equiv n_{1}, n_{2}, \ldots, n_{r}$ of the boring eMZVs on the left-hand side are deconcatenated $^{10}$ into smaller tuples $A_{j}=a_{1}^{j}, a_{2}^{j}, \ldots, a_{m_{j}}^{j}$ such that all eMZVs $\omega\left(A_{j}\right)$ are interesting. Only even numbers of interesting $\omega\left(A_{j}\right)$ are compatible with the boring nature of $\omega(B)$, and the concatenation $A_{j} A_{j+1}$ in equation (A.1) is defined to yield $a_{1}^{j}, \ldots, a_{m_{j}}^{j}, a_{1}^{j+1}, \ldots, a_{m_{j+1}}^{j+1}$.

Note that the first appearance of $D_{4}=-\frac{1}{8}$ can be seen from the second case $\omega\left(n_{1}, n_{2}, n_{3}, n_{4}\right)$ in equation (2.20). The vanishing of eMZVs with all entries odd (see equation (2.21)) follows from the absence of deconcatenations into tuples $A_{j}$ with $\omega\left(A_{j}\right)$ interesting.

In order to prove ${ }^{11}$ equations (A.1) and (A.2), we recall that the antipode

$$
\mathcal{S}\left(n_{1}, n_{2}, \ldots, n_{r}\right) \equiv(-1)^{r}\left(n_{r}, \ldots, n_{2}, n_{1}\right)
$$

in the shuffle algebra of words $B=n_{1}, n_{2}, \ldots, n_{r}$ satisfies the following defining property [51]

$$
B+\mathcal{S}(B)+\sum_{\substack{B=A_{1} A_{2} \\ A_{1}, A_{2} \neq \varnothing}} A_{1} w \mathcal{S}\left(A_{2}\right)=0, \quad B \neq \varnothing .
$$

\footnotetext{
10 For example, the $k=1$ part of equation (A.1) encompasses those deconcatenations $B=A_{1} A_{2}$ into $A_{1}=n_{1}, n_{2}, \ldots, n_{j}$ and $A_{2}=n_{j+1}, \ldots, n_{r}$ where $\omega\left(n_{1}, n_{2}, \ldots, n_{j}\right)$ and $\omega\left(n_{j+1}, \ldots, n_{r}\right)$ are interesting eMZVs.

11 We are grateful to an anonymous referee for suggesting the proof.
} 
Since boring and interesting eMZVs can be neatly characterized through the antipode equation (A.3),

$$
\omega(\mathcal{S}(B))= \begin{cases}\omega(B) & : \omega(B) \text { boring } \\ -\omega(B) & : \omega(B) \text { interesting }\end{cases}
$$

applying $\omega(\cdot)$ to (A.4) with boring $\omega(B)$ yields

$$
\left.\omega(B)\right|_{\text {boring }}=\frac{1}{2}\left\{\sum_{\substack{B=A_{1} A_{2} \\ \omega\left(A_{i}\right) \text { interesting }}} \omega\left(A_{1}\right) \omega\left(A_{2}\right)-\sum_{\substack{B=B_{1} B_{2} \\ \omega\left(B_{i}\right) \text { boring }}} \omega\left(B_{1}\right) \omega\left(B_{2}\right)\right\} .
$$

This formula can be recursively applied to the boring factors $\omega\left(B_{i}\right)$ on the right-hand side until only interesting contributions remain, leading to the structure of equation (A.1). The coefficients $D_{2 k}$ in equation (A.2) are determined by the combinatorics of iterating equation (A.6), e.g.

$$
\begin{aligned}
\left.\omega(B)\right|_{\text {boring }}= & \frac{1}{2} \sum_{\substack{B=A_{1} A_{2} \\
\omega\left(A_{i}\right) \text { interesting }}} \omega\left(A_{1}\right) \omega\left(A_{2}\right) \\
& -\frac{1}{8} \sum_{\substack{B=B_{1} B_{2} \\
\omega\left(B_{i}\right) \text { boring }}}\left\{\sum_{\substack{B_{1}=A_{1} A_{2} \\
\omega\left(A_{i}\right) \text { interesting }}} \omega\left(A_{1}\right) \omega\left(A_{2}\right)-\sum_{\substack{B_{1}=B_{3} B_{4} \\
\omega\left(B_{i}\right) \text { boring }}} \omega\left(B_{3}\right) \omega\left(B_{4}\right)\right\} \\
& \times\left\{\begin{array}{c}
\left.\sum_{\substack{B_{2}=A_{3} A_{4} \\
\omega\left(A_{i}\right) \text { interesting }}} \omega\left(A_{3}\right) \omega\left(A_{4}\right)-\sum_{\substack{B_{2}=B_{5} B_{6} \\
\omega\left(B_{i}\right) \text { boring }}} \omega\left(B_{5}\right) \omega\left(B_{6}\right)\right\}
\end{array}\right.
\end{aligned}
$$

reproduces the first two terms with $k \leqslant 2$ in equation (A.1). In the next iteration step towards $k=3$, either the first boring pair $\omega\left(B_{3}\right) \omega\left(B_{4}\right)$ or the second one $\omega\left(B_{5}\right) \omega\left(B_{6}\right)$ can be rearranged via equation (A.6). Since both of them contribute $\frac{1}{32} \sum_{B=A_{1} \ldots A_{6}} \omega\left(A_{1}\right) \ldots \omega\left(A_{6}\right)$ with $\omega\left(A_{i}\right)$ interesting, the above coefficient $D_{6}=\frac{1}{16}$ rests on the two subdivisions of the schematic form $\left\{\left(A_{1} A_{2}\right)\left(A_{3} A_{4}\right)\right\}\left(A_{5} A_{6}\right)$ or $\left(A_{1} A_{2}\right)\left\{\left(A_{3} A_{4}\right)\left(A_{5} A_{6}\right)\right\}$, referring to the application of equation (A.6) to either $\omega\left(B_{3}\right) \omega\left(B_{4}\right)$ or $\omega\left(B_{5}\right) \omega\left(B_{6}\right)$, respectively.

The contributions of $\sum_{B=A_{1} \ldots A_{2 k}}$ from the iteration of equation (A.6) can be organized in terms of full binary trees with $k-1$ internal vertices and $k$ leaves. Internal vertices represent the expansion of pairs of boring eMZVs via equation (A.6) and at each of the $k$ leaves, a pair of interesting eMZVs is kept. Hence, the coefficient of $\sum_{B=A_{1} \ldots A_{2 k}} \omega\left(A_{1}\right) \ldots \omega\left(A_{2 k}\right)$ in equation (A.1) is given by

$$
D_{2 k}=\frac{1}{2}\left(-\frac{1}{4}\right)^{k-1} \cdot \#(\text { full binary trees with k leaves })
$$

where each additional application of equation (A.6) to a pair of boring $\omega\left(B_{i}\right)$ involves a prefactor of $-\frac{1}{4}$. Finally, since full binary trees with $k$ leaves are counted by the Catalan number $C_{k-1}[52]$ with

$$
C_{n}=\frac{(2 n) !}{(n+1) ! n !}=2^{n} \frac{(2 n-1) ! !}{(n+1) !},
$$

we recover the coefficients $D_{2 k}=\frac{(-1)^{k-1}}{2^{2 k-1}} C_{k-1}$ in equation (A.2) from equation (A.8). 


\section{A.2. More general Fay identities}

The relation equation (2.25) among elliptic iterated integrals yields various Fay identities in the limit $z \rightarrow 1$ and generalizes as follows to multiple appearances of the argument among the labels:

$$
\begin{aligned}
& \Gamma\left(\begin{array}{lllll}
n_{1} & n_{2} \ldots n_{k} & n_{k+1} & \ldots & n_{r} \\
z & z \ldots z & 0 & \ldots & 0
\end{array} ; z\right)=(-1)^{k} \zeta^{\omega}(\underbrace{0 \ldots 0}_{r-k} \underbrace{1 \ldots 1}_{k}) \prod_{j=1}^{r} \delta_{n_{j}, 1} \\
& -(-1)^{n_{k}} \int_{0}^{z} \mathrm{~d} t f^{\left(n_{k}+n_{k+1}\right)}(t) \Gamma\left(\begin{array}{lllllll}
n_{1} & \ldots & n_{k-1} & 0 & n_{k+2} & \ldots & n_{r} \\
t & \ldots & t & 0 & 0 & \ldots & 0
\end{array} ;\right) \\
& +\sum_{j=0}^{n_{k+1}}\left(\begin{array}{c}
n_{k}-1+j \\
j
\end{array}\right) \int_{0}^{z} \mathrm{~d} t f^{\left(n_{k+1}-j\right)}(t) \Gamma\left(\begin{array}{lllllll}
n_{1} & \ldots & n_{k-1} & n_{k}+j & n_{k+2} & \ldots & n_{r} \\
t & \ldots & t & t & 0 & \ldots & 0
\end{array} ;\right) \\
& +\sum_{j=0}^{n_{k}}\left(\begin{array}{c}
n_{k+1}-1+j \\
j
\end{array}\right)(-1)^{n_{k}+j} \\
& \times \int_{0}^{z} \mathrm{~d} t f^{\left(n_{k}-j\right)}(t) \Gamma\left(\begin{array}{lllllll}
n_{1} & \ldots & n_{k-1} & n_{k+1}+j & n_{k+2} & \ldots & n_{r} \\
t & \ldots & t & 0 & 0 & \ldots & 0
\end{array} ; t\right) .
\end{aligned}
$$

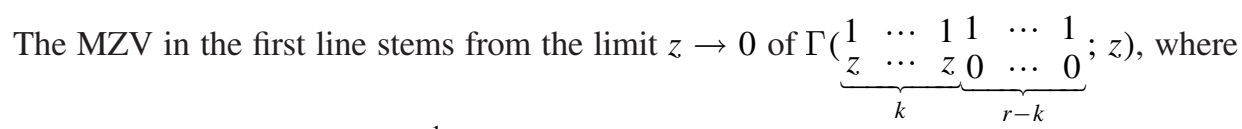
every $f^{(1)}(z)$ can be replaced by $\frac{1}{z}$ in this regime. As explained in [5], the elliptic iterated integrals then reduce to particular instances of multiple polylogarithms, which can be shown to yield MZVs in this case.

The first novel eMZV relations follow from the limit $z \rightarrow 1$ of equation (A.10) at $k=2$ and $r=4,5$ :

$$
\begin{aligned}
& \Gamma\left(\begin{array}{llll}
n_{1} & n_{2} & n_{3} & n_{4} \\
z & z & 0 & 0
\end{array} ; z\right) \\
& =-\frac{1}{4} \zeta_{4} \delta_{n_{1}, 1} \delta_{n_{2}, 1} \delta_{n_{3}, 1} \delta_{n_{4}, 1}-(-1)^{n_{2}} \\
& \times \int_{0}^{z} \mathrm{~d} t f^{\left(n_{2}+n_{3}\right)}(t) \Gamma\left(\begin{array}{lll}
n_{1} & 0 & n_{4} \\
t & 0 & 0
\end{array}\right) ; t \\
& +\sum_{j=0}^{n_{3}}\left(\begin{array}{c}
n_{2}-1+j \\
j
\end{array}\right) \int_{0}^{z} \mathrm{~d} t f^{\left(n_{3}-j\right)}(t) \Gamma\left(\begin{array}{lll}
n_{1} & n_{2}+j & n_{4} \\
t & t & 0
\end{array} ;\right) \\
& +\sum_{j=0}^{n_{2}}\left(\begin{array}{c}
n_{3}-1+j \\
j
\end{array}\right)(-1)^{n_{2}+j} \int_{0}^{z} \mathrm{~d} t f^{\left(n_{2}-j\right)}(t) \Gamma\left(\begin{array}{lll}
n_{1} & n_{3}+j & n_{4} \\
t & 0 & 0
\end{array} ;\right) \\
& \Gamma\left(\begin{array}{lllll}
n_{1} & n_{2} & n_{3} & n_{4} & n_{5} \\
z & z & 0 & 0 & 0
\end{array} ; z\right) \\
& =\left(2 \zeta_{5}-\zeta_{2} \zeta_{3}\right) \times\left(\prod_{j=1}^{5} \delta_{n_{j}, 1}\right)-(-1)^{n_{2}} \int_{0}^{z} \mathrm{~d} t f^{\left(n_{2}+n_{3}\right)}(t) \Gamma\left(\begin{array}{llll}
n_{1} & 0 & n_{4} & n_{5} \\
t & 0 & 0 & 0
\end{array} ;\right)
\end{aligned}
$$




$$
\begin{aligned}
& +\sum_{j=0}^{n_{3}}\left(\begin{array}{c}
n_{2}-1+j \\
j
\end{array}\right) \int_{0}^{z} \mathrm{~d} t f^{\left(n_{3}-j\right)}(t) \Gamma\left(\begin{array}{llll}
n_{1} & n_{2}+j & n_{4} & n_{5} \\
t & t & 0 & 0
\end{array}\right) \\
& +\sum_{j=0}^{n_{2}}\left(\begin{array}{c}
n_{3}-1+j \\
j
\end{array}\right)(-1)^{n_{2}+j} \int_{0}^{z} \mathrm{~d} t f^{\left(n_{2}-j\right)}(t) \Gamma\left(\begin{array}{llll}
n_{1} & n_{3}+j & n_{4} & n_{5} \\
t & 0 & 0 & 0
\end{array} ;\right)
\end{aligned}
$$

In particular, note that the product $\zeta_{2} \zeta_{3}$ is absent in equation (2.25) at $r=5$. Also, note that the divergent nature of $f^{(1)}$ causes extra complications in the limit $z \rightarrow 1$ of equation (A.11) if $n_{i}=1$ for $i=1,2,3,4$ and equation (A.12) if $n_{2}=n_{3}=n_{4}=1$ and one of $n_{1}=1$ or $n_{5}=1$.

\section{Appendix B. Iterated Eisenstein integrals versus eMZVs: examples}

In this appendix, we supplement further examples for the conversion of eMZVs into modified iterated Eisenstein integrals as defined in equation (4.54).

\section{B.1. Conversion of $\omega(0,0, \ldots, 0, n)$}

For eMZVs with only one non-zero entry, a closed formula can be given for their conversion into iterated Eisenstein integrals. At length $\ell_{\omega}=4$ and $\ell_{\omega}=5$, equations (4.58a) and (4.58b) can be generalized to

$$
\begin{aligned}
\omega(0,0,0, n)=\delta_{n, 1}\left(\frac{\mathrm{i} \pi}{12}+\frac{\zeta_{3}}{4 \pi^{2}}\right) & +\frac{n}{3 !} \gamma_{0}(n+1)+n(n+1)(n+2) \gamma_{0}(n+3,0,0) \\
\omega(0,0,0,0, n)= & -\frac{2 \zeta_{n}}{5 !}-\frac{n}{3 !}(n+1) \gamma_{0}(n+2,0) \\
& -n(n+1)(n+2)(n+3) \gamma_{0}(n+4,0,0,0),
\end{aligned}
$$

where $n$ is chosen to be odd in equation (B.1) and even in equation (B.2). At arbitrary length $\ell$, we have

$$
\begin{aligned}
& \omega(\underbrace{0,0, \ldots, 0}_{\ell-1}, n) \\
& =\left\{\begin{array}{cl}
\omega_{0}\left(0^{\ell-1}, n\right)+\sum_{\substack{i=1,3,5, \ldots, \ell-1}} \frac{\gamma_{0}\left(n+i, 0^{i-1}\right)}{(\ell-i) !} \prod_{j=0}^{i-1}(n+j): \ell \text { even, } n \text { odd } \\
-\frac{2 \zeta_{n}}{\ell !}-\sum_{\substack{i=2,4,6, \ldots, \ell-1}} \frac{\gamma_{0}\left(n+i, 0^{i-1}\right)}{(\ell-i) !} \prod_{j=0}^{i-1}(n+j) & : \ell \text { odd, } n \text { even }
\end{array},\right.
\end{aligned}
$$

where the constant term for odd values of $n$ vanishes except for weight one,

$$
\omega_{0}\left(0^{\ell-1}, n\right)=\delta_{n, 1}\left\{\frac{\mathrm{i} \pi}{2(\ell-1) !}-\sum_{k=1}^{\lfloor\ell / 2\rfloor-1} \frac{(-1)^{k}}{(\ell-(2 k+1)) !} \frac{\zeta_{2 k+1}}{\left(4 \pi^{2}\right)^{k}}\right\} .
$$


The $q$-expansion of equation (B.3) can be inferred from the special case of equation (4.64),

$$
\gamma_{0}\left(k, 0^{p-1}\right)=-\frac{2(2 \pi \mathrm{i})^{k-2 p}}{(k-1) !} \sum_{n=1}^{\infty} \frac{\sigma_{k-1}(n) q^{n}}{n^{p}},
$$

see equation (4.63) for the definition of the divisor sum $\sigma_{k}(n)$.

\section{B.2. Conversion of indecomposable eMZVs at $\ell_{\omega} \geqslant 3$}

Among the indecomposable eMZVs beyond $\omega(0, \ldots, 0, n)$, the simplest case $\omega(0,3,5)$ is converted to (modified) iterated Eisenstein integrals in equations (4.15) and (4.61). Beyond that, we find for example

$$
\begin{aligned}
\omega(0,3,7)= & -1848 \gamma(12,0)-294 \gamma(8,4)+\mathrm{nmt} \\
= & -1848 \gamma_{0}(12,0)-294 \gamma_{0}(8,4)-75\left(\gamma_{0}(6)\right)^{2} \\
& +588 \zeta_{4} \gamma_{0}(8,0)-504 \zeta_{8} \gamma_{0}(4,0) \\
\omega(0,3,9)= & -5616 \gamma(14,0)-729 \gamma(10,4)-315 \gamma(8,6)+\mathrm{nmt} \\
= & -5616 \gamma_{0}(14,0)-729 \gamma_{0}(10,4)-315 \gamma_{0}(8,6) \\
& -210 \gamma_{0}(6) \gamma_{0}(8)+1458 \zeta_{4} \gamma_{0}(10,0)+630 \zeta_{6} \gamma_{0}(8,0)-630 \zeta_{6} \gamma_{0}(6,0) \\
& -1350 \zeta_{10} \gamma_{0}(4,0) \\
\omega(0,3,11)= & -13695 \gamma(16,0)-1452 \gamma(12,4)-990 \gamma(10,6)+\mathrm{nmt} \\
= & -13695 \gamma_{0}(16,0)-1452 \gamma_{0}(12,4)-\frac{735}{2}\left(\gamma_{0}(8)\right)^{2} \\
& -990 \gamma_{0}(10,6)-270 \gamma_{0}(6) \gamma_{0}(10)+2904 \zeta_{4} \gamma_{0}(12,0) \\
& +1980 \zeta_{6} \gamma_{0}(10,0)-1980 \zeta_{10} \gamma_{0}(6,0)-2772 \zeta_{12} \gamma_{0}(4,0) \\
= & -30105 \gamma(16,0)-5445 \gamma(12,4)-3105 \gamma(10,6)+\mathrm{nmt} \\
\omega(0,5,9) & -30105 \gamma_{0}(16,0)-5445 \gamma_{0}(12,4) \\
& -3105 \gamma_{0}(10,6)-\frac{735}{2}\left(\gamma_{0}(8)\right)^{2}+10890 \zeta_{4} \gamma_{0}(12,0)+6210 \zeta_{6} \gamma_{0}(10,0) \\
& -5850 \zeta_{10} \gamma_{0}(6,0)-8910 \zeta_{12} \gamma_{0}(4,0)
\end{aligned}
$$

(B.6)

at length three, and

$$
\begin{aligned}
\omega(0,0,2,3)= & 252 \gamma(8,0,0)-18 \gamma(4,4,0)+\frac{5}{6} \gamma(6)+\mathrm{nmt} \\
= & 252 \gamma_{0}(8,0,0)-18 \gamma_{0}(4,4,0)+\frac{5}{6} \gamma_{0}(6)-72 \zeta_{4} \gamma_{0}(4,0,0) \\
\omega(0,0,2,5)= & 2826 \gamma(10,0,0)+150 \gamma(6,4,0)+180 \gamma(6,0,4)+\frac{7}{6} \gamma(8)+\mathrm{nmt} \\
= & 2826 \gamma_{0}(10,0,0)+150 \gamma_{0}(6,4,0)+180 \gamma_{0}(6,0,4)+\frac{7}{6} \gamma_{0}(8) \\
& -660 \zeta_{4} \gamma_{0}(6,0,0)+180 \zeta_{6} \gamma_{0}(4,0,0) \\
\omega(0,0,4,3)= & 2340 \gamma(10,0,0)-300 \gamma(6,4,0) \\
& -120 \gamma(6,0,4)+\frac{7}{6} \gamma(8)+\mathrm{nmt} \\
= & -2340 \gamma_{0}(10,0,0)-300 \gamma_{0}(6,4,0) \\
& -120 \gamma_{0}(6,0,4)-60 \gamma_{0}(4) \gamma_{0}(6,0)+\frac{7}{6} \gamma_{0}(8) \\
& +480 \zeta_{4} \gamma_{0}(6,0,0)-1080 \zeta_{6} \gamma_{0}(4,0,0)-3 \zeta_{4} \gamma_{0}(4)
\end{aligned}
$$


at length four, where 'nmt' refers to non-maximal terms as explained after equation (4.17a). The $q$-expansion of the constituents is given by equation (4.64).

\section{Appendix C. Examples for relations in the derivation algebra ॥}

\section{C.1. Known relations}

Irreducible relations $r_{\text {weight }}^{\text {depth }}$ are listed in table 5. For depth two, all relations can be obtained from equation (4.42). At depth three, we can confirm the relations listed in equation (4.28c) as well as [24]:

$$
\begin{array}{rl}
r_{20}^{3}:=0 & 1050\left[\epsilon_{0},\left[\epsilon_{6}, \epsilon_{14}\right]\right]-6580\left[\epsilon_{0},\left[\epsilon_{8}, \epsilon_{12}\right]\right] \\
& +4320\left[\epsilon_{4},\left[\epsilon_{0}, \epsilon_{16}\right]\right]-10970\left[\epsilon_{4},\left[\epsilon_{4}, \epsilon_{12}\right]\right] \\
& +166675\left[\epsilon_{4},\left[\epsilon_{6}, \epsilon_{10}\right]\right]-17150\left[\epsilon_{6},\left[\epsilon_{0}, \epsilon_{14}\right]\right] \\
& -500675\left[\epsilon_{6},\left[\epsilon_{6}, \epsilon_{8}\right]\right]+30184\left[\epsilon_{8},\left[\epsilon_{0}, \epsilon_{12}\right]\right] \\
& +80388\left[\epsilon_{8},\left[\epsilon_{4}, \epsilon_{8}\right]\right]-17325\left[\epsilon_{10},\left[\epsilon_{0}, \epsilon_{10}\right]\right]
\end{array}
$$

$r_{22}^{3}: \quad 0=40\left[\epsilon_{0},\left[\epsilon_{6}, \epsilon_{16}\right]\right]-280\left[\epsilon_{0},\left[\epsilon_{8}, \epsilon_{14}\right]\right]$

$+910\left[\epsilon_{0},\left[\epsilon_{10}, \epsilon_{12}\right]\right]-360\left[\epsilon_{4},\left[\epsilon_{0}, \epsilon_{18}\right]\right]$

$-11535\left[\epsilon_{4},\left[\epsilon_{6}, \epsilon_{12}\right]\right]+6069\left[\epsilon_{4},\left[\epsilon_{8}, \epsilon_{10}\right]\right]+1320\left[\epsilon_{6},\left[\epsilon_{0}, \epsilon_{16}\right]\right]$

$+15140\left[\epsilon_{6},\left[\epsilon_{4}, \epsilon_{12}\right]\right]-7150\left[\epsilon_{6},\left[\epsilon_{6}, \epsilon_{10}\right]\right]-1820\left[\epsilon_{8},\left[\epsilon_{0}, \epsilon_{14}\right]\right]$

$-12922\left[\epsilon_{8},\left[\epsilon_{6}, \epsilon_{8}\right]\right]+858\left[\epsilon_{10},\left[\epsilon_{0}, \epsilon_{12}\right]\right]$

$r_{18}^{4}: \quad 0=\left[\epsilon_{0},\left[\epsilon_{0},\left[\epsilon_{6}, \epsilon_{12}\right]\right]\right]-\frac{215}{74}\left[\epsilon_{0},\left[\epsilon_{0},\left[\epsilon_{8}, \epsilon_{10}\right]\right]\right]-\frac{2323}{518}\left[\epsilon_{0},\left[\epsilon_{4},\left[\epsilon_{6}, \epsilon_{8}\right]\right]\right]$

$+\frac{218}{37}\left[\epsilon_{0},\left[\epsilon_{6},\left[\epsilon_{4}, \epsilon_{8}\right]\right]\right]+\frac{60}{407}\left[\epsilon_{4},\left[\epsilon_{0},\left[\epsilon_{0}, \epsilon_{14}\right]\right]\right]$

$+\frac{285561}{5698}\left[\epsilon_{4},\left[\epsilon_{0},\left[\epsilon_{6}, \epsilon_{8}\right]\right]\right]+\frac{8599}{1628}\left[\epsilon_{4},\left[\epsilon_{4},\left[\epsilon_{0}, \epsilon_{10}\right]\right]\right]$

$+\frac{53855}{444}\left[\epsilon_{4},\left[\epsilon_{4},\left[\epsilon_{4}, \epsilon_{6}\right]\right]\right]$

$-\frac{691}{333}\left[\epsilon_{6},\left[\epsilon_{0},\left[\epsilon_{0}, \epsilon_{12}\right]\right]\right]-\frac{19853}{518}\left[\epsilon_{6},\left[\epsilon_{0},\left[\epsilon_{4}, \epsilon_{8}\right]\right]\right]$

$-\frac{691}{74}\left[\epsilon_{10},\left[\epsilon_{0},\left[\epsilon_{0}, \epsilon_{8}\right]\right]\right]$

$+\frac{691}{111}\left[\epsilon_{12},\left[\epsilon_{0},\left[\epsilon_{0}, \epsilon_{6}\right]\right]\right]-\frac{60}{37}\left[\epsilon_{14},\left[\epsilon_{0},\left[\epsilon_{0}, \epsilon_{4}\right]\right]\right]$

$-\frac{87595}{1554}\left[\epsilon_{6},\left[\epsilon_{4},\left[\epsilon_{0}, \epsilon_{8}\right]\right]\right]$

$+\frac{17275}{333}\left[\epsilon_{6},\left[\epsilon_{6},\left[\epsilon_{0}, \epsilon_{6}\right]\right]\right]+\frac{3455}{518}\left[\epsilon_{8},\left[\epsilon_{0},\left[\epsilon_{0}, \epsilon_{10}\right]\right]\right]$

$+\frac{49565}{518}\left[\epsilon_{8},\left[\epsilon_{0},\left[\epsilon_{4}, \epsilon_{6}\right]\right]\right]$ 


$$
\begin{aligned}
& r_{22}^{4}: \quad 0=\left[\epsilon_{0},\left[\epsilon_{0},\left[\epsilon_{8}, \epsilon_{14}\right]\right]\right]+\frac{192903}{230}\left[\epsilon_{0},\left[\epsilon_{4},\left[\epsilon_{6}, \epsilon_{12}\right]\right]\right] \\
& -\frac{861492}{805}\left[\epsilon_{0},\left[\epsilon_{6},\left[\epsilon_{4}, \epsilon_{12}\right]\right]\right]+\frac{134488}{161}\left[\epsilon_{0},\left[\epsilon_{6},\left[\epsilon_{6}, \epsilon_{10}\right]\right]\right] \\
& +\frac{6588}{805}\left[\epsilon_{4},\left[\epsilon_{0},\left[\epsilon_{0}, \epsilon_{18}\right]\right]\right] \\
& +\frac{269217}{805}\left[\epsilon_{4},\left[\epsilon_{0},\left[\epsilon_{6}, \epsilon_{12}\right]\right]\right]-\frac{39418}{115}\left[\epsilon_{4},\left[\epsilon_{0},\left[\epsilon_{8}, \epsilon_{10}\right]\right]\right] \\
& -\frac{13253}{115}\left[\epsilon_{4},\left[\epsilon_{4},\left[\epsilon_{0}, \epsilon_{14}\right]\right]\right] \\
& -\frac{18221}{115}\left[\epsilon_{4},\left[\epsilon_{4},\left[\epsilon_{6}, \epsilon_{8}\right]\right]\right]+\frac{33109}{322}\left[\epsilon_{6},\left[\epsilon_{0},\left[\epsilon_{6}, \epsilon_{10}\right]\right]\right] \\
& +\frac{25095129}{37375}\left[\epsilon_{6},\left[\epsilon_{4},\left[\epsilon_{0}, \epsilon_{12}\right]\right]\right] \\
& +\frac{11266827}{5750}\left[\epsilon_{6},\left[\epsilon_{4},\left[\epsilon_{4}, \epsilon_{8}\right]\right]\right]-\frac{786557}{644}\left[\epsilon_{6},\left[\epsilon_{6},\left[\epsilon_{0}, \epsilon_{10}\right]\right]\right] \\
& +\frac{80233}{1265}\left[\epsilon_{8},\left[\epsilon_{0},\left[\epsilon_{0}, \epsilon_{14}\right]\right]\right] \\
& +\frac{21742068}{6325}\left[\epsilon_{8},\left[\epsilon_{0},\left[\epsilon_{6}, \epsilon_{8}\right]\right]\right]-\frac{112835}{253}\left[\epsilon_{8},\left[\epsilon_{4},\left[\epsilon_{0}, \epsilon_{10}\right]\right]\right] \\
& +\frac{403764}{115}\left[\epsilon_{8},\left[\epsilon_{4},\left[\epsilon_{4}, \epsilon_{6}\right]\right]\right] \\
& +\frac{644938}{575}\left[\epsilon_{8},\left[\epsilon_{6},\left[\epsilon_{0}, \epsilon_{8}\right]\right]\right]-\frac{103859}{115}\left[\epsilon_{10},\left[\epsilon_{0},\left[\epsilon_{4}, \epsilon_{8}\right]\right]\right] \\
& +\frac{301851}{8050}\left[\epsilon_{12},\left[\epsilon_{0},\left[\epsilon_{0}, \epsilon_{10}\right]\right]\right] \\
& +\frac{734133}{805}\left[\epsilon_{12},\left[\epsilon_{0},\left[\epsilon_{4}, \epsilon_{6}\right]\right]\right]-\frac{493889}{8050}\left[\epsilon_{10},\left[\epsilon_{0},\left[\epsilon_{0}, \epsilon_{12}\right]\right]\right] \\
& -\frac{372888}{10465}\left[\epsilon_{6},\left[\epsilon_{0},\left[\epsilon_{0}, \epsilon_{16}\right]\right]\right] \\
& -\frac{23054063}{52325}\left[\epsilon_{6},\left[\epsilon_{0},\left[\epsilon_{4}, \epsilon_{12}\right]\right]\right]-\frac{1015637}{1150}\left[\epsilon_{0},\left[\epsilon_{4},\left[\epsilon_{8}, \epsilon_{10}\right]\right]\right] \\
& -\frac{27458211}{3220}\left[\epsilon_{6},\left[\epsilon_{6},\left[\epsilon_{4}, \epsilon_{6}\right]\right]\right] \\
& -\frac{23679}{8050}\left[\epsilon_{0},\left[\epsilon_{0},\left[\epsilon_{10}, \epsilon_{12}\right]\right]\right]-\frac{1913}{115}\left[\epsilon_{14},\left[\epsilon_{0},\left[\epsilon_{0}, \epsilon_{8}\right]\right]\right] \\
& +\frac{672}{115}\left[\epsilon_{16},\left[\epsilon_{0},\left[\epsilon_{0}, \epsilon_{6}\right]\right]\right] \\
& -\frac{972}{805}\left[\epsilon_{18},\left[\epsilon_{0},\left[\epsilon_{0}, \epsilon_{4}\right]\right]\right]
\end{aligned}
$$

\section{C.2. New relations}

At depth 5 we explicitly isolated the irreducible relation $r_{20}^{5}$, which is apparently new:

$r_{20}^{5}: 0$

$=2206388620800\left[\epsilon_{0},\left[\epsilon_{0},\left[\epsilon_{0},\left[\epsilon_{4}, \epsilon_{16}\right]\right]\right]\right]$

$-8366188740000\left[\epsilon_{0},\left[\epsilon_{0},\left[\epsilon_{0},\left[\epsilon_{6}, \epsilon_{14}\right]\right]\right]\right]$ 
$+12305858292000\left[\epsilon_{0},\left[\epsilon_{0},\left[\epsilon_{0},\left[\epsilon_{8}, \epsilon_{12}\right]\right]\right]\right]$

$-1834700544000\left[\epsilon_{0},\left[\epsilon_{4},\left[\epsilon_{0},\left[\epsilon_{0}, \epsilon_{16}\right]\right]\right]\right]$

$+35687825530800\left[\epsilon_{0},\left[\epsilon_{4},\left[\epsilon_{0},\left[\epsilon_{4}, \epsilon_{12}\right]\right]\right]\right]$

$-109425220173750\left[\epsilon_{0},\left[\epsilon_{4},\left[\epsilon_{0},\left[\epsilon_{6}, \epsilon_{10}\right]\right]\right]\right]$

- $39970750599360\left[\epsilon_{0},\left[\epsilon_{4},\left[\epsilon_{4},\left[\epsilon_{0}, \epsilon_{12}\right]\right]\right]\right]$

$-380488416808500\left[\epsilon_{0},\left[\epsilon_{4},\left[\epsilon_{4},\left[\epsilon_{4}, \epsilon_{8}\right]\right]\right]\right]$

$+13171256280000\left[\epsilon_{0},\left[\epsilon_{6},\left[\epsilon_{0},\left[\epsilon_{0}, \epsilon_{14}\right]\right]\right]\right]$

$+220479512028750\left[\epsilon_{0},\left[\epsilon_{6},\left[\epsilon_{0},\left[\epsilon_{4}, \epsilon_{10}\right]\right]\right]\right]$

$-498847136287500\left[\epsilon_{0},\left[\epsilon_{6},\left[\epsilon_{0},\left[\epsilon_{6}, \epsilon_{8}\right]\right]\right]\right]$

$+220479512028750\left[\epsilon_{0},\left[\epsilon_{6},\left[\epsilon_{4},\left[\epsilon_{0}, \epsilon_{10}\right]\right]\right]\right]$

$-458212979593200\left[\epsilon_{0},\left[\epsilon_{6},\left[\epsilon_{4},\left[\epsilon_{4}, \epsilon_{6}\right]\right]\right]\right]$

$+17540335312500\left[\epsilon_{0},\left[\epsilon_{6},\left[\epsilon_{6},\left[\epsilon_{0}, \epsilon_{8}\right]\right]\right]\right]$

$-34407225652800\left[\epsilon_{0},\left[\epsilon_{8},\left[\epsilon_{0},\left[\epsilon_{0}, \epsilon_{12}\right]\right]\right]\right]$

- $97419791414400\left[\epsilon_{0},\left[\epsilon_{8},\left[\epsilon_{0},\left[\epsilon_{4}, \epsilon_{8}\right]\right]\right]\right]$

$-197536749664800\left[\epsilon_{0},\left[\epsilon_{8},\left[\epsilon_{4},\left[\epsilon_{0}, \epsilon_{8}\right]\right]\right]\right]$

$+22970739577500\left[\epsilon_{0},\left[\epsilon_{10},\left[\epsilon_{0},\left[\epsilon_{0}, \epsilon_{10}\right]\right]\right]\right]$

$+161385266688750\left[\epsilon_{0},\left[\epsilon_{10},\left[\epsilon_{0},\left[\epsilon_{4}, \epsilon_{6}\right]\right]\right]\right]$

$+611566848000\left[\epsilon_{4},\left[\epsilon_{0},\left[\epsilon_{0},\left[\epsilon_{0}, \epsilon_{16}\right]\right]\right]\right]$

$-58836403790864\left[\epsilon_{4},\left[\epsilon_{0},\left[\epsilon_{0},\left[\epsilon_{4}, \epsilon_{12}\right]\right]\right]\right]$

$+134572047805000\left[\epsilon_{4},\left[\epsilon_{0},\left[\epsilon_{0},\left[\epsilon_{6}, \epsilon_{10}\right]\right]\right]\right]$

$+9658664444426884\left[\epsilon_{4},\left[\epsilon_{0},\left[\epsilon_{4},\left[\epsilon_{4}, \epsilon_{8}\right]\right]\right]\right]$

$+92810063342256\left[\epsilon_{4},\left[\epsilon_{4},\left[\epsilon_{0},\left[\epsilon_{0}, \epsilon_{12}\right]\right]\right]\right]$

$-204658497503460\left[\epsilon_{4},\left[\epsilon_{4},\left[\epsilon_{0},\left[\epsilon_{4}, \epsilon_{8}\right]\right]\right]\right]$

$+541534390897500\left[\epsilon_{4},\left[\epsilon_{4},\left[\epsilon_{4},\left[\epsilon_{0}, \epsilon_{8}\right]\right]\right]\right]$

$-215755493216250\left[\epsilon_{4},\left[\epsilon_{6},\left[\epsilon_{0},\left[\epsilon_{0}, \epsilon_{10}\right]\right]\right]\right]$

$-1490371718737200\left[\epsilon_{4},\left[\epsilon_{6},\left[\epsilon_{0},\left[\epsilon_{4}, \epsilon_{6}\right]\right]\right]\right]$

$+1032598095322950\left[\epsilon_{4},\left[\epsilon_{6},\left[\epsilon_{4},\left[\epsilon_{0}, \epsilon_{6}\right]\right]\right]\right]$

$+298655975581600\left[\epsilon_{4},\left[\epsilon_{8},\left[\epsilon_{0},\left[\epsilon_{0}, \epsilon_{8}\right]\right]\right]\right]$

$-220479512028750\left[\epsilon_{4},\left[\epsilon_{10},\left[\epsilon_{0},\left[\epsilon_{0}, \epsilon_{6}\right]\right]\right]\right]$

$+54837332264496\left[\epsilon_{4},\left[\epsilon_{12},\left[\epsilon_{0},\left[\epsilon_{0}, \epsilon_{4}\right]\right]\right]\right]$

- $6941740260000\left[\epsilon_{6},\left[\epsilon_{0},\left[\epsilon_{0},\left[\epsilon_{0}, \epsilon_{14}\right]\right]\right]\right]$

$-220479512028750\left[\epsilon_{6},\left[\epsilon_{0},\left[\epsilon_{0},\left[\epsilon_{4}, \epsilon_{10}\right]\right]\right]\right]$

$+231883232831250\left[\epsilon_{6},\left[\epsilon_{0},\left[\epsilon_{0},\left[\epsilon_{6}, \epsilon_{8}\right]\right]\right]\right]$

$+519528504682200\left[\epsilon_{6},\left[\epsilon_{0},\left[\epsilon_{4},\left[\epsilon_{4}, \epsilon_{6}\right]\right]\right]\right]$

$-220479512028750\left[\epsilon_{6},\left[\epsilon_{4},\left[\epsilon_{0},\left[\epsilon_{0}, \epsilon_{10}\right]\right]\right]\right]$

$-2120947122294000\left[\epsilon_{6},\left[\epsilon_{4},\left[\epsilon_{0},\left[\epsilon_{4}, \epsilon_{6}\right]\right]\right]\right]$

$-1538522546497950\left[\epsilon_{6},\left[\epsilon_{4},\left[\epsilon_{4},\left[\epsilon_{0}, \epsilon_{6}\right]\right]\right]\right]$

$+249423568143750\left[\epsilon_{6},\left[\epsilon_{6},\left[\epsilon_{0},\left[\epsilon_{0}, \epsilon_{8}\right]\right]\right]\right]$

$-266963903456250\left[\epsilon_{6},\left[\epsilon_{8},\left[\epsilon_{0},\left[\epsilon_{0}, \epsilon_{6}\right]\right]\right]\right]$

$+23162632092600\left[\epsilon_{8},\left[\epsilon_{0},\left[\epsilon_{0},\left[\epsilon_{0}, \epsilon_{12}\right]\right]\right]\right]$

$+184988881773150\left[\epsilon_{8},\left[\epsilon_{0},\left[\epsilon_{0},\left[\epsilon_{4}, \epsilon_{8}\right]\right]\right]\right]$

$+310347440367510\left[\epsilon_{8},\left[\epsilon_{4},\left[\epsilon_{0},\left[\epsilon_{0}, \epsilon_{8}\right]\right]\right]\right]$

$-183822714075000\left[\epsilon_{8},\left[\epsilon_{6},\left[\epsilon_{0},\left[\epsilon_{0}, \epsilon_{6}\right]\right]\right]\right]$ 


$$
\begin{aligned}
& +171943360038450\left[\epsilon_{8},\left[\epsilon_{8},\left[\epsilon_{0},\left[\epsilon_{0}, \epsilon_{4}\right]\right]\right]\right] \\
& -22551859687500\left[\epsilon_{10},\left[\epsilon_{0},\left[\epsilon_{0},\left[\epsilon_{0}, \epsilon_{10}\right]\right]\right]\right] \\
& -240755752121625\left[\epsilon_{10},\left[\epsilon_{0},\left[\epsilon_{0},\left[\epsilon_{4}, \epsilon_{6}\right]\right]\right]\right] \\
& -104628710038125\left[\epsilon_{10},\left[\epsilon_{4},\left[\epsilon_{0},\left[\epsilon_{0}, \epsilon_{6}\right]\right]\right]\right] \\
& -14987648446875\left[\epsilon_{10},\left[\epsilon_{6},\left[\epsilon_{0},\left[\epsilon_{0}, \epsilon_{4}\right]\right]\right]\right] \\
& +11918038532400\left[\epsilon_{12},\left[\epsilon_{0},\left[\epsilon_{0},\left[\epsilon_{0}, \epsilon_{8}\right]\right]\right]\right] \\
& +46293152724000\left[\epsilon_{12},\left[\epsilon_{4},\left[\epsilon_{0},\left[\epsilon_{0}, \epsilon_{4}\right]\right]\right]\right] \\
& -8366188740000\left[\epsilon_{14},\left[\epsilon_{0},\left[\epsilon_{0},\left[\epsilon_{0}, \epsilon_{6}\right]\right]\right]\right] .
\end{aligned}
$$

The complete set of all irreducible relations known to us is available from https://tools.aei. mpg.de/emzv, and all of them have been verified by evaluating the action on the letters $x, y$ via equation $(4.26 a)$.

\section{References}

[1] Enriquez B 2013 Analogues elliptiques des nombres multizétas arxiv:1301.3042

[2] Levin A 1997 Elliptic polylogarithms: an analytic theory Compos. Math. 106267

[3] Levin A and Racinet G 2007 Towards multiple elliptic polylogarithms arxiv:math/0703237

[4] Brown F and Levin A 2011 Multiple elliptic polylogarithms arxiv:1110.6917v2

[5] Broedel J, Mafra C R, Matthes N and Schlotterer O 2015 Elliptic multiple zeta values and oneloop superstring amplitudes J. High Energy Phys. JHEP07(2015)112

[6] Bloch S and Vanhove P 2015 The elliptic dilogarithm for the sunset graph J. Number Theory 148328

[7] Adams L, Bogner C and Weinzierl S 2014 The two-loop sunrise graph in two space-time dimensions with arbitrary masses in terms of elliptic dilogarithms J. Math. Phys. 55102301

[8] Bloch S, Kerr M and Vanhove P 2014 A Feynman integral via higher normal functions arxiv: 1406.2664

[9] Adams L, Bogner C and Weinzierl S 2015 The two-loop sunrise integral around four space-time dimensions and generalisations of the Clausen and Glaisher functions towards the elliptic case J. Math. Phys. 56072303

[10] Søgaard M and Zhang Y 2015 Elliptic functions and maximal unitarity Phys. Rev. D 91081701

[11] Brown F C S 2012 On the decomposition of motivic multiple zeta values Galois-Teichmüller Theory and Arithmetic Geometry (Tokyo: Math. Soc. Japan) pp 31-58

[12] Brown F 2012 Mixed Tate motives over $\mathbb{Z}$ Ann. Math. 175949

[13] Broadhurst D J and Kreimer D 1997 Association of multiple zeta values with positive knots via Feynman diagrams up to 9 loops Phys. Lett. B 393403

[14] Zagier D 1994 Values of zeta functions and their applications First European Congress of Mathematics vol II (Basel: Birkhäuser) pp 497-512 (Paris, 1992)

[15] Blumlein J, Broadhurst D and Vermaseren J 2010 The Multiple zeta value data mine Comput. Phys. Commun. 181582

[16] Enriquez B 2014 Elliptic associators Sel. Math. (N.S.) 20491

[17] Manin Y I 2006 Iterated integrals of modular forms and noncommutative modular symbols Algebraic Geometry and Number Theory (Berlin: Springer) pp 565-597

[18] Brown F 2014 Multiple modular values for $\mathrm{SL}_{2}(\mathbb{Z})$ arxiv:1407.5167v1

[19] Gangl H, Kaneko M and Zagier D 2006 Double zeta values and modular forms Automorphic Forms and Zeta Functions (Hackensack, NJ: World Scientific) pp 71-106

[20] Hain R 2014 The Hodge-de R̃ham theory of modular groups arxiv:1403.6443

[21] Matthes N Work in progress

[22] Calaque D, Enriquez B and Etingof P 2009 Universal KZB equations: the elliptic case Algebra, Arithmetic, and Geometry: in Honor of Yu I Manin vol I (Boston MA: Birkhäuser Boston, Inc.,) pp 165-266

[23] Hain R 2013 Notes on the universal elliptic KZB equation arxiv:1309.0580

Q4 [24] Pollack A Relations between derivations arising from modular forms Undergraduate Thesis Duke University 
[25] Brown F 2015 Zeta elements in depth 3 and the fundamental Lie algebra of a punctured elliptic curve arxiv: 1504.04737

[26] Luque J-G, Novelli J-C and Thibon J-Y 2006 Period polynomials and Ihara brackets arXiv: math/ 0606301

[27] Kronecker L 1881 Zur theorie der elliptischen funktionen Mathematische Werke IV p 313

[28] Mumford D, Nori M and Norman P 1983 Tata Lectures on Theta I, II (Basel: Birkhäuser) (1984)

[29] Matthes N 2015 Elliptic double zeta values arxiv:1509.08760

Q6 [30] Drinfeld V G 1989 Quasi-Hopf algebras Algebra Analiz 1114

[31] Drinfeld V 1991 On quasitriangular quasi-Hopf algebras and on a group that is closely connected with $\operatorname{Gal}(\overline{\mathbb{Q}} / \mathbb{Q})$ Leningrad Math. J. 2829

[32] Le T and Murakami J 1996 Kontsevich's integral for the Kauffman polynomial Nagoya Math. J. 14293

[33] Racinet G 2002 Doubles mélanges des polylogarithmes multiples aux racines de l'unité Publ. Math. Inst. Hautes Études Sci. 185

[34] Apéry R and de Irrationalité $1979 \zeta(2)$ et $\zeta(3)$ Astérisque 6111

[35] Ball K and Rivoal T 2001 Irrationalité d'une infinité de valeurs de la fonction zeta aux entiers impairs Invent. Math. 146193

[36] Goncharov A B 2005 Galois symmetries of fundamental groupoids and noncommutative geometry Duke Math. J. 128209

[37] Brown F 2014 Motivic periods and the projective line minus three points Proc. ICM 2014 (arxiv:1407.5165)

[38] Schlotterer O and Stieberger S 2013 Motivic Multiple zeta values and superstring amplitudes J. Phys. A: Math. Theor. 46475401

[39] Deligne P 1989 Le groupe fondamental de la droite projective moins trois points Galois Groups Over Q (Berkeley, CA, 1987) (New York: Springer) pp 79-297 (1987)

[40] Baumard S and Schneps L 2013 Relations dans l'algèbre de Lie fondamentale des motifs elliptiques mixtes arxiv: 1310.5833

[41] Baumard S and Schneps L 2015 On the derivation representation of the fundamental Lie algebra of mixed elliptic motives arxiv:1510.05549

[42] http://oeis.org

[43] Brown F Letter to Nils Matthes

[44] Drummond J and Ragoucy E 2013 Superstring amplitudes and the associator J. High Energy Phys. JHEP08(2013)135

[45] Broedel J, Schlotterer O, Stieberger S and Terasoma T 2014 All order $\alpha^{\prime}$-expansion of superstring trees from the Drinfeld associator Phys. Rev. D 89066014

[46] Green M B and Vanhove P 2000 The Low-energy expansion of the one loop type II superstring amplitude Phys. Rev. D 61104011

[47] Green M B, Russo J G and Vanhove P 2008 Low energy expansion of the four-particle genus-one amplitude in type II superstring theory J. High Energy Phys. JHEP02(2008)020

[48] D'Hoker E, Green M B and Vanhove P 2015 On the modular structure of the genus-one type II superstring low energy expansion J. High Energy Phys. JHEP08(2015)041

[49] Richards D M 2008 The one-loop five-graviton amplitude and the effective action J. High Energy Phys. JHEP10(2008)042

[50] Green M B, Mafra C R and Schlotterer O 2013 Multiparticle one-loop amplitudes and S-duality in closed superstring theory J. High Energy Phys. JHEP10(2013)188

[51] Abe E 1980 Hopf Algebras (Cambridge: Cambridge University Press) p xii+284 Translated from the Japanese by Hisae Kinoshita and Hiroko Tanaka

[52] Stanley R P 1999 Enumerative Combinatorics vol 2 (Cambridge: Cambridge University Press) p xii +581 With a foreword by Gian-Carlo Rota and appendix 1 by Sergey Fomin 


\section{QUERY FORM}

JouRnal: Journal of Physics A: Mathematical and Theoretical

Author: J Broedel et al

TITLE: Relations between elliptic multiple zeta values and a special derivation algebra

ARTiCle ID: jpaaa10c0

The layout of this article has not yet been finalized. Therefore this proof may contain columns that are not fully balanced/matched or overlapping text in inline equations; these issues will be resolved once the final corrections have been incorporated.

We have been provided funding information for this article as below. Please confirm whether this information is correct. European Research Council: ERC Advanced Grant No. 247252, Michael Green.

\section{Page 1}

Q1

Please specify the corresponding author.

Page 48

Q2

Please check the details for any journal references that do not have a link as they may contain some incorrect information.

\section{Page 48}

Q3

Please provide updated details for references [1, 3, 4, 8, 18, 20, 21, 23, 25, 26, 29, 40, 41, 43] if available.

\section{Page 48}

Q4

Thesis references should contain author name, year, type of thesis and institution. Please provide the missing information in reference [24].

\section{Page 49}

Q5

Publisher location and name are required for book reference [27]. Please provide the missing information. 
Page 49

Q6

Please provide the page range or article number in references [30, 33]. 